Anna C. Ulpe*, Katharina C.L. Bauerfeind, Luis I. Granone, Arsou Arimi, Lena Megatif, Ralf Dillert, Sven Warfsmann, Dereje H. Taffa, Michael Wark, Detlef W. Bahnemann and Thomas Bredow

\title{
Photoelectrochemistry of Ferrites: Theoretical Predictions vs. Experimental Results
}

https://doi.org/10.1515/zpch-2019-1449

Received April 15, 2019; accepted November 19, 2019

Abstract: This paper gives an overview about recent theoretical and experimental work on electronic and optical properties of spinel ferrites $\mathrm{MFe}_{2} \mathrm{O}_{4}$. These compounds have come into focus of research due to their possible application as photocatalyst material for photoelectrochemical water splitting. The theoretical background of state-of-the-art quantum-chemical approaches applied for predicting electronic and optical band gaps, absolute band positions, optical absorption spectra, dielectric functions and Raman spectra, is briefly reviewed. Recent applications of first-principles methods on magnetic and electronic properties of

*Corresponding author: Anna C. Ulpe, Mulliken Center for Theoretical Chemistry, Institut für Physikalische und Theoretische Chemie, Universität Bonn, Beringstraße 4-6, D-53115 Bonn, Germany, e-mail: anna@thch.uni-bonn.de

Katharina C.L. Bauerfeind and Thomas Bredow: Mulliken Center for Theoretical Chemistry, Institut für Physikalische und Theoretische Chemie, Universität Bonn, Beringstraße 4-6, D-53115 Bonn, Germany

Luis I. Granone, Arsou Arimi and Lena Megatif: Institut für Technische Chemie, Gottfried Wilhelm Leibniz Universität Hannover, Callinstraße 3, D-30167 Hannover, Germany Ralf Dillert: Institut für Technische Chemie, Gottfried Wilhelm Leibniz Universität Hannover, Callinstraße 3, D-30167 Hannover, Germany; and Laboratorium für Nano- und Quantenengineering, Gottfried Wilhelm Leibniz Universität Hannover, Schneiderberg 39, D-30167 Hannover, Germany

Sven Warfsmann, Dereje H. Taffa and Michael Wark: Institute of Chemistry, Chemical Technology 1, Carl von Ossietzky University Oldenburg, Carl-von-Ossietzky-Str. 9-11, D-26129 Oldenburg, Germany

Detlef W. Bahnemann: Institut für Technische Chemie, Gottfried Wilhelm Leibniz Universität Hannover, Callinstraße 3, D-30167 Hannover, Germany; and Laboratory 'Photoactive Nanocomposite Materials', Saint-Petersburg State University, Ulyanovskaya Str. 1, Peterhof, Saint-Petersburg, 198504 Russia 
ferrites with $M=\mathrm{Mg}$ and the first row of subgroup elements Sc to $\mathrm{Zn}$ are presented, where it is shown that the fundamental band gap is strongly dependent on the spin state and the degree of inversion of the spinel structure. The observed variation of electronic properties may serve as an explanation for the large scattering of experimental results. The exchange of $M$ and Fe cations has also a pronounced effect on the Raman spectra of ferrites, which is analyzed at atomic scale from first principles. Calculated optical absorption spectra of ferrites are compared to experimental spectra. The electronic nature of the first excitations and the role of oxygen vacancies are discussed. For the calculation of absolute band positions, which have a significant impact on the photoelectrochemical activity of the ferrites, models of the most stable ferrite surfaces are developed that take into account their polar nature and the interaction with the solvent. Theoretically predicted valence and conduction band edges are compared to results from electrochemical measurements. The role of cation exchange on the surface electronic structure is investigated both theoretically and experimentally.

Keywords: DFT; perturbation theory; photoelectrochemical water splitting; spectroscopy; spinel ferrites.

PACS: 82.65.+r; 71.15.Mb; 77.84.Bw; 78.20.-e; 78.30.-j.

\section{Introduction}

Because of the urgent need for an exit from nuclear and fossil energy sources, the research on new sustainable ways for energy production is indispensable. Among others, molecular hydrogen is considered as substitute for fossil fuels. The remaining challenge is its cost efficient production, e.g. by solar powered water splitting.

Photoelectrochemical (PEC) water splitting is a promising tool for the conversion of solar energy into molecular hydrogen under mild operating conditions [1]. The PEC water splitting makes use of semiconductor materials with an optimal band gap of $1.2 \mathrm{eV}$ plus $\sim 0.8 \mathrm{eV}$ overvoltage as electrode material.

Spinel ferrites $\left(\mathrm{MFe}_{2} \mathrm{O}_{4}\right)$ have been discussed as suitable electrode material for photoelectrochemical water splitting $[1,2]$. Spinel ferrites are ternary transition metal oxides adopting the spinel structure (space group Fd3̄m). Ferrites with $M=\mathrm{Mg}, \mathrm{Mn}, \mathrm{Fe}, \mathrm{Co}, \mathrm{Ni}, \mathrm{Cu}, \mathrm{Zn}$ are frequently discussed [2], while those with $M=\mathrm{Sc}, \mathrm{Ti}, \mathrm{V}, \mathrm{Cr}$ are scarcely investigated. Depending on the substituent $M$ and the synthesis conditions, spinel ferrites can adopt different cation distributions. In general, their chemical formula can be written as $\left[M_{1-x} \mathrm{Fe}_{x}\right]^{A}\left[M_{x} \mathrm{Fe}_{2-x}\right]^{B} \mathrm{O}_{4}$. The superscripts $A$ and $B$ denote the tetrahedral (8a) and octahedral (16d) sites, 
respectively, and $x$ corresponds to the degree of inversion. $x$ ranges between 0 and 1 , where $x=0$ represents a so-called 'normal' and $x=1$ an 'inverse' spinel.

State-of-the-art theory enables to investigate properties that are difficult to access by experiments. These are, for example, the characterization of compounds that are difficult to synthesize, the detailed electronic structure and its changes by the influence of magnetic structure, defects or doping. This information is crucial to optimize the performance of a material.

A large variety of theoretical methods is available for the investigation of electronic and optical properties, and they will be discussed briefly in the next section. In detail, we will discuss several extensions of density functional theory (DFT), e.g. DFT + U, dynamical mean field theory (DMFT) and different versions of Hybrid DFT, as well as $G W$ and Bethe-Salpeter approaches.

Previous theoretical work on spinel ferrites was often based on the DFT $+\mathrm{U}$ approximation [3-29]. The most frequently investigated ferrite using DFT $+\mathrm{U}$ approaches is probably $\mathrm{Fe}_{3} \mathrm{O}_{4}$. But not only the high-temperature spinel phase of $\mathrm{Fe}_{3} \mathrm{O}_{4}$ is of interest $[3,16,23,24]$, also different low-temperature phases have been subject of DFT+U studies [4, 5, 14], and also the phase transition between both phases was investigated [6]. An influence of cation distribution on the electronic properties is demonstrated using DFT $+\mathrm{U}$ for $\mathrm{ZnFe}_{2} \mathrm{O}_{4}$ and $\mathrm{CdFe}_{2} \mathrm{O}_{4}$ by Cheng and Liu [7] and for $\mathrm{CuFe}_{2} \mathrm{O}_{4}$ by Feng et al. [8]. Fritsch and Ederer show that a treatment of $\mathrm{CoFe}_{2} \mathrm{O}_{4}$ and $\mathrm{NiFe}_{2} \mathrm{O}_{4}$ with $\mathrm{DFT}+\mathrm{U}$ yields qualitatively correct results for the electronic structure $[9,17]$. The energetics of different cation distributions calculated with $\mathrm{DFT}+\mathrm{U}$ for $\mathrm{CoFe}_{2} \mathrm{O}_{4}$ show, that $\mathrm{CoFe}_{2} \mathrm{O}_{4}$ favors the inverse spinel structure and a high-spin configuration [10]. Corresponding investigations were carried out for $\mathrm{MnFe}_{2} \mathrm{O}_{4}$, showing a preference for a normal cation distribution and a high-spin configuration [19], as well as a strong support for a single-valence state of $\mathrm{Mn}^{2+}$ [20]. Several works use DFT $+\mathrm{U}$ to investigate the effects of doping, like Mn doping in $\mathrm{CdFe}_{2} \mathrm{O}_{4}$ [12], $\mathrm{B}$ incorporation in $\mathrm{NiFe}_{2} \mathrm{O}_{4}$ [25], or $\mathrm{Ca}$ and $\mathrm{Ba}$ doping in $\mathrm{Fe}_{3} \mathrm{O}_{4}$ [28]. Furthermore, the investigation of spinel surfaces and the adsorption of small molecules has been subject of DFT + U studies, e.g. the adsorption of $\mathrm{NO}$ on the $\mathrm{CuFe}_{2} \mathrm{O}_{4}$ (100) surface [13] or the adsorption of a single $\mathrm{Au}$ atom on $\mathrm{Fe}_{3} \mathrm{O}_{4}$ (111) [16]. Kumar et al. investigate surface reactivity and water adsorption on $\mathrm{NiFe}_{2} \mathrm{O}_{4}$ (111) and find that the surface reactivity of $\mathrm{NiFe}_{2} \mathrm{O}_{4}$ (111) is higher compared to $\mathrm{Fe}_{3} \mathrm{O}_{4}$ (111) [22]. The investigation of atomic structure, electronic properties and reactivity of the $\mathrm{NiFe}_{2} \mathrm{O}_{4}$ (001) surface by Shi et al. shows, that oxygen vacancies form preferably on the (001) surface compared to the bulk [26]. Liu et al. recently applied self-consistent charge density functional tight binding (SCC-DFTB) to investigate magnetite bulk and low index surfaces [30]. They state that the results are in quantitative agreement with those obtained by DFT $+\mathrm{U}$ and hybrid DFT methods. 
Hybrid functionals have also found application for spinel ferrites [15, 23, 29, 31, 32]. Rowan and Patterson investigate the electronic structures and vibrational modes of the low-temperature and high-temperature phases of $\mathrm{Fe}_{3} \mathrm{O}_{4}$ using hybrid-DFT calculations [31]. They observe major changes in the Raman active modes when $\mathrm{Fe}_{3} \mathrm{O}_{4}$ undergoes the phase transition. Sun et al. combined LSDA+U and hybrid DFT calculations for the investigation of $\mathrm{NiFe}_{2} \mathrm{O}_{4}$ [15]. By evaluating projected density of states, they show a qualitative agreement between both methods. $\mathrm{NiFe}_{2} \mathrm{O}_{4}$ is shown to be an indirect band gap material, with the lowestenergy electronic transition being assigned to the minority channel. Evaluation of the states near the band gap reveals a $p-d$ and $d-d$ charge transfer character in both channels. Widatallah et al. investigate Mg doping in lithium ferrite [32]. By investigating 19 defect structures, they find that it is most stable when $\mathrm{Mg}^{2+}$ is evenly replacing $\mathrm{Li}^{+}$and $\mathrm{Fe}^{3+}$. Noh et al. compare GGA, GGA+U and range separated hybrid-DFT results on $\mathrm{Fe}_{3} \mathrm{O}_{4}$ [23]. They show that the inclusion of exact exchange has a significant impact on the electronic structure. The HSE06 functional predicts highly localized states below the Fermi level of $\mathrm{Fe}_{3} \mathrm{O}_{4}$, while the same functional with reduced amount of Fock exchange (15\%) and GGA+U predict delocalized states around the Fermi level. Fritsch carried out a detailed investigation on the influence of the degree of inversion and different magnetic structures on the electronic and magnetic properties of $\mathrm{ZnFe}_{2} \mathrm{O}_{4}$ [29]. Comparing the performance of GGA $+\mathrm{U}$ and the hybrid-DFT functional PBE0, he shows that PBEO yields an overall better agreement with experiment.

$G W$ methods have scarcely been applied to spinel ferrites so far. Ziaei and Bredow applied $G W$-BSE theory to compute optical properties of $\mathrm{ZnFe}_{2} \mathrm{O}_{4}$ [33]. It was shown that the valence band maximum is dominated by oxygen $2 p$-orbitals, whereas the conduction band minimum consists of hybridized oxygen $2 p$ - and iron $3 d$-orbitals.

Here, we present hybrid-DFT and GW/BSE studies on electronic and optical properties of $M \mathrm{Fe}_{2} \mathrm{O}_{4}$ with $M=\mathrm{Mg}$, Sc-Zn. By the use of dielectric dependent self-consistent hybrid DFT, we studied the influence of cation distribution and spin state on the electronic band gap and the nature of the lowest lying electronic transitions. Hybrid DFT was applied for the calculation of Raman spectra for $\mathrm{ZnFe}_{2} \mathrm{O}_{4}$ to further evaluate the influence of cation inversion on the material properties. Optical spectra for different cation distributions and magnetic structures of $\mathrm{ZnFe}_{2} \mathrm{O}_{4}$ were computed using $\mathrm{GW}$-BSE methods. The dependency of absolute band positions on the cation distribution in $\mathrm{ZnFe}_{2} \mathrm{O}_{4}$ (100) surface models was investigated at hybrid DFT level.

The theoretical predictions reviewed in this work are compared with experimental results found in literature and our own measurements. For $\mathrm{ZnFe}_{2} \mathrm{O}_{4}$ in particular, the calculated Raman and optical spectrum, and absolute band positions 
are compared to those obtained experimentally. Special emphasis was placed on comparing experimental and theoretical results concerning the effect of the degree of inversion and deviations from ideal stoichiometry on the electronic and optical properties of $\mathrm{ZnFe}_{2} \mathrm{O}_{4}$.

\section{Theoretical approaches}

In this section we present the most common methods and theoretical models that are used to calculate electronic and optical properties of solids and surfaces.

\subsection{Methods}

\subsubsection{DFT}

Density-Functional Theory (DFT) relies on findings by Hohenberg and Kohn in 1964 [34] that prove a connection between the exact ground-state energy of an $N$-electron system and its electron density $n(\boldsymbol{r})$ of the form

$$
n(\boldsymbol{r})=N \int\left|\Psi\left(\boldsymbol{r}_{1}, \boldsymbol{r}_{2}, \ldots, \boldsymbol{r}_{N}\right)\right|^{2} d \boldsymbol{r}_{2}, \ldots, d \boldsymbol{r}_{N}
$$

The DFT energy functional can be written as

$$
E_{\mathrm{KS}-\mathrm{DFT}}[n(\boldsymbol{r})]=E_{e n}[n(\boldsymbol{r})]+T[n(\boldsymbol{r})]+E_{e e}[n(\boldsymbol{r})],
$$

where $E_{\text {en }}[n(\boldsymbol{r})]$ is the electron-core interaction, $T[n(\boldsymbol{r})]$ the kinetic energy of the electrons, and $E_{e e}[(n(r)]$ is the electron-electron interaction. Since the exact energy functional is unknown, simplifications have to be made in order to solve the equation. In Kohn-Sham(KS)-DFT [35], the exact kinetic energy of the electrons $T$ is replaced by a kinetic energy of non-interacting electrons. The electronelectron interaction is usually divided in two terms:

$$
E_{e e}[n(\boldsymbol{r})]=J[n(\boldsymbol{r})]+E_{x c}[n(\boldsymbol{r})]
$$

$J[n(\boldsymbol{r})]$ is the electron-electron Coulomb repulsion and the exchangecorrelation energy $E_{x c}[n(\boldsymbol{r})]$ is the unknown part of the energy, that remains when we subtract the kinetic energy of non-interacting electrons and the Coulomb terms $E_{e n}$ and $J$ from the exact energy. The unknown $E_{x c}$ is approximated differently by common DFT functionals. For further information on the approximation of $E_{x c}$, the reader is referred to the references [35] and [36]. 
An important part in the electron density of $E_{x c}$ is the so-called exchangecorrelation hole, that represents a 'hole', that each electron creates around itself as a consequence of exchange-correlation effects. One of the main problems of the KS model occurs when the energy of virtual orbitals is required, as for the calculation of band gaps. The virtual KS orbitals represent excited electrons in the KS reference system. They feel the same potential as the occupied orbitals, that is the Coulomb potential of $N$ electrons and an exchange-correlation hole of $(N-1)$ electrons. The virtual orbitals are artificially stabilized by this exchangecorrelation hole and the KS fundamental band gap becomes too small [37].

The description of electronic states using DFT is further limited by its singledeterminantal nature. The wavefunctions of systems containing partially filled $d$ or $f$ shells of transition metals have a multi-determinantal character. An accurate description can be obtained e.g. with complete active space (CASSCF) methods [38]. While this is possible for small molecules, there is no generally applicable CASSCF implementation for solids available at present.

A widely applied approximation that is also used in the present study is the broken symmetry approach. The initial guess for an unrestricted Kohn-Sham wavefunction is constructed from atomic wavefunctions with certain orbital occupation numbers and magnetic states. In this way ferro-, ferri-, and antiferromagnetic states can be approximated by a single reference wavefunction.

\subsubsection{DFT+U}

$\mathrm{DFT}+\mathrm{U}$ is widely applied for electronic structure calculations where plain DFT fails. This is generally the case when strongly correlated electrons are present, e.g. for transition metal oxides with partially filled $d$ or $f$ shells. To address this problem, electrons are separated into delocalized $s$ and $p$ electrons, that can be described by standard DFT, and localized $d$ or $f$ electrons, for which intraatomic two-electron interaction integrals have to be taken into account. Anisimov et al. [39] introduced an on-site Hubbard-like correction $E_{\mathrm{Hub}}$ to the standard LDA functional

$$
E_{\mathrm{LDA}+\mathrm{U}}[n(\boldsymbol{r})]=E_{\mathrm{LDA}}[n(\boldsymbol{r})]+E_{\mathrm{Hub}}\left[\left\{n_{m}^{\sigma}\right\}\right]-E_{\mathrm{DC}}\left[\left\{n^{\sigma}\right\}\right],
$$

with the electronic density $n(\boldsymbol{r})$, and elements of the density matrix $\left\{n_{m}^{\sigma}\right\}$ with magnetic quantum number $m$ and the spin index $\sigma$. The term $E_{\mathrm{DC}}$ subtracts double counting of the interactions that are covered by both, $E_{\mathrm{LDA}}$ and $E_{\mathrm{Hub}}$. 
A rotationally invariant version to calculate $E_{\mathrm{Hub}}$ was introduced in 1995 [40, 41]:

$$
\begin{aligned}
E_{\mathrm{Hub}}[\{n\}]= & \frac{1}{2} \sum_{\{m\}, \sigma}\left\{\left\langle m, m^{\prime \prime}\left|V_{e e}\right| m^{\prime}, m^{\prime \prime \prime}\right\rangle n_{m m^{\prime}}^{\sigma} n_{m^{\prime \prime} m^{\prime \prime \prime}}^{-\sigma}\right. \\
& +\left(\left\langle m, m^{\prime \prime}\left|V_{e e}\right| m^{\prime}, m^{\prime \prime \prime}\right\rangle\right. \\
& \left.\left.-\left\langle m, m^{\prime \prime}\left|V_{e e}\right| m^{\prime \prime \prime}, m^{\prime}\right\rangle\right) n_{m m^{\prime}}^{\sigma} n_{m^{\prime \prime} m^{\prime \prime \prime}}^{\sigma}\right\},
\end{aligned}
$$

with the screened Coulomb interactions $V_{e e}$ between $d$ or $f$ electrons and

$$
\left\langle m, m^{\prime \prime}\left|V_{e e}\right| m^{\prime}, m^{\prime \prime \prime}\right\rangle=\sum_{k=0}^{2 l} a_{k}\left(m, m^{\prime}, m^{\prime \prime}, m^{\prime \prime \prime}\right) F^{k}
$$

Here, $l$ is the orbital quantum number of the localized $d$ or $f$ electrons and $a_{k}$ can be expressed in terms of spherical harmonics:

$$
a_{k}\left(m, m^{\prime}, m^{\prime \prime}, m^{\prime \prime \prime}\right)=\frac{4 \pi}{2 k+1} \sum_{q=-k}^{k}\left\langle\operatorname{lm}\left|Y_{k q}\right| \operatorname{lm}{ }^{\prime}\right\rangle \times\left\langle\operatorname{lm}^{\prime \prime}\left|Y_{k q}^{\star}\right| \operatorname{lm} m^{\prime \prime \prime}\right\rangle .
$$

$F^{k}$ are effective Slater integrals that can be expressed in terms of the screened on-site Coulomb interaction $U$ and the screened exchange interaction $J$ as $U=F^{0}$ and $J=\left(F^{2}+F^{4}\right) / 14$. To simplify the procedure, often an 'effective' Hubbard potential $U_{\text {eff }}=U-J$ is used. In practice, $U_{\text {eff }}$ is often adjusted in a semiempirical way in order to reproduce certain properties of a specific compound.

More recently, Cococcioni and de Gironcoli proposed an internally consistent calculation of the Hubbard potential associated to site I [42]:

$$
U_{\text {eff }}=\left(\chi_{0}^{-1}-\chi^{-1}\right)_{I I}
$$

This formulation makes use of the density response functions $\chi$

$$
\begin{aligned}
& \chi_{I J}=\frac{\partial E}{\partial \alpha_{I} \partial \alpha_{J}}=\frac{\partial n_{I}}{\partial \alpha_{J}} \\
& \chi_{I J}^{0}=\frac{\partial^{2} E^{\mathrm{KS}}}{\partial \alpha_{I}^{\mathrm{KS}} \partial \alpha_{J}^{\mathrm{KS}}}=\frac{\partial n_{I}}{\partial \alpha_{I}^{\mathrm{KS}}},
\end{aligned}
$$

where $\alpha_{I}$ are Lagrange multipliers. 


\subsubsection{Hybrid DFT}

The idea of hybrid DFT is to mix exact Fock exchange and DFT exchange, in order to reduce the deficiencies of DFT functionals introduced by the self-interaction error. Hybrid functionals were first introduced by Axel Becke in 1993 [43] and reformulated in 1996 [44] to

$$
E_{x c}^{\text {hybrid }}=E_{x c}^{\mathrm{DFT}}+a_{0} \cdot\left(E_{\chi}^{\text {Fock }}-E_{x}^{\mathrm{DFT}}\right) .
$$

$a_{0}$ is a constant fraction of exact exchange and is dependent on the material and the underlying DFT functional.

In order to take into account screening effects, the amount of exact exchange can be made dependent on the distance between electrons $r$. Range separated hybrid (RSH) functionals make use of this by partition of the Coulomb operator in the so-called 'short-range' (SR), 'middle-range' (MR), and 'long-range' (LR) regions [45, 46]. This can be accomplished using the error functions [46]:

$$
\frac{1}{r}=\frac{\operatorname{erfc}\left(\omega_{\mathrm{SR}} r\right)}{r}+\frac{\operatorname{erfc}\left(\omega_{\mathrm{LR}} r\right)-\operatorname{erfc}\left(\omega_{\mathrm{SR}} r\right)}{r}+\frac{\operatorname{erf}\left(\omega_{\mathrm{LR}} r\right)}{r} .
$$

$\omega_{\mathrm{SR}}$ and $\omega_{\mathrm{LR}}$ are parameters defining the range of separation. The exchangecorrelation energy of a RSH functional can generally be written as

$$
E_{x c}^{\mathrm{RSH}}=E_{x c}^{\mathrm{DFT}}+c_{\mathrm{SR}}\left(E_{x, \mathrm{SR}}^{\mathrm{Fock}}-E_{x, \mathrm{SR}}^{\mathrm{DFT}}\right)+c_{\mathrm{MR}}\left(E_{x, \mathrm{MR}}^{\mathrm{Fock}}-E_{x, \mathrm{MR}}^{\mathrm{DFT}}\right)+c_{\mathrm{LR}}\left(E_{x, \mathrm{LR}}^{\mathrm{Fock}}-E_{x, \mathrm{LR}}^{\mathrm{DFT}}\right) .
$$

The coefficients $c_{\mathrm{SR}}, c_{\mathrm{MR}}, c_{\mathrm{LR}}$ as well as the parameters $\omega_{\mathrm{SR}}$ and $\omega_{\mathrm{LR}}$ determine the nature of the RSH.

Hybrid functionals greatly improved the accuracy of DFT, yet this ansatz is not parameter free. Recently, the material dependency of the exchange fraction $a_{0}$ was linked to the static dielectric constant [47] as

$$
a_{0}=\frac{1}{\varepsilon}
$$

The dielectric constant can either be taken from experiment or calculated from first principles. Erba suggested a fully-automated computationally-efficient implementation calculating $a_{0}$ self-consistently from the dielectric constant by adopting a Coupled-Perturbed-Hartree-Fock/Kohn-Sham (CPHF/KS) approach [48] (cf. Section 2.2).

\subsubsection{GW/BSE}

As Baerendts pointed out [37], Kohn-Sham single-particle energies can not be interpreted as ionization energies and electron affinities. The DFT orbital energies 
can be corrected using the $G W$ approximation within many-body perturbation theory, which describes quasiparticle (QP) states with the exchange correlation self-energy $\Sigma$ [49]:

$$
H_{0}(\boldsymbol{r}) \Psi_{i}(\boldsymbol{r}, \omega)+\int d^{3} r \Sigma\left(\boldsymbol{r}, \boldsymbol{r}^{\prime}, \omega\right) \Psi_{i}\left(\boldsymbol{r}^{\prime}, \omega\right)=E_{i}(\omega) \Psi_{i}(\boldsymbol{r}, \omega) .
$$

In the $G W$ approximation, the self-energy operator[50] has the form

$$
\Sigma\left(\boldsymbol{r}, \boldsymbol{r}^{\prime}, \omega\right)=\frac{i}{4 \pi} \int_{-\infty}^{\infty} e^{i \omega^{\prime} \delta} G\left(\boldsymbol{r}, \boldsymbol{r}^{\prime}, \omega+\omega^{\prime}\right) W\left(\boldsymbol{r}, \boldsymbol{r}^{\prime}, \omega^{\prime}\right) d \omega,
$$

which is a product of the Green's function $G$ and the screened Coulomb interaction $W$. The Green's function in its spectral representation [49] can be written as

$$
G\left(\boldsymbol{r}, \boldsymbol{r}^{\prime}, \omega\right)=\sum_{i} \frac{\Psi_{i}(\boldsymbol{r}, \omega) \Psi_{i}^{\dagger}\left(\boldsymbol{r}^{\prime}, \omega\right)}{\omega-E_{i}(\omega)} .
$$

The index $i$ is a combination of the band and $k$-point indices. $\Psi_{i}$ again are the solutions to the QP equation (14).

The dynamically screened Coulomb interaction [50] can be evaluated as

$$
W_{\boldsymbol{q}}\left(\boldsymbol{G}, \boldsymbol{G}^{\prime}, \omega\right)=4 \pi e^{2} \frac{1}{|\boldsymbol{q}+\boldsymbol{G}|} \boldsymbol{\varepsilon}_{\boldsymbol{q}}^{-1}\left(\boldsymbol{G}, \boldsymbol{G}^{\prime}, \omega\right) \frac{1}{\left|\boldsymbol{q}+\boldsymbol{G}^{\prime}\right|} .
$$

This is a product of the bare Coulomb kernel and the inverse dielectric matrix $\boldsymbol{\varepsilon} . \boldsymbol{G}$ is a reciprocal lattice vector and $\boldsymbol{q}$ a Bloch wave vector. The dielectric matrix can be obtained within the random phase approximation and is connected to the time-ordered independent-particle polarizability $\chi$. For further information on the evaluation of $\boldsymbol{\varepsilon}$ and $\boldsymbol{\chi}$, the reader is referred e.g. to reference [50].

Based on the fundamental equation (14), the initial guess for $G$ and $W$ is obtained from mean field methods, e.g. LDA or GGA. If only one iteration is performed, the approach is called one-shot $G W$ or $G_{0} W_{0}$. To further increase accuracy, partially or fully self-consistent approaches can be used. In partially self-consistent approaches, $G$ is iterated self-consistently, while the dynamically screened interaction $W$ is kept at the mean-field level. This procedure is often denoted as $G W_{0}$. The evaluation of $G$ can either be for the quasiparticle energies only (evGW $W_{0}$ ), or for both, eigenvalues and wavefunctions (sc $G W_{0}$ ). In fully self-consistent approaches, iterations in $G$ and $W$ are performed. As for partially self-consistent schemes, two versions, evGW and scGW, can be distinguished.

To describe optical excitation spectra, the single-particle picture in $G W$ is not sufficient. For the description of electron-hole interaction, the equation of motion 
of the two-particle Green's function has to be evaluated. This formulation, also known as the Bethe-Salpeter equation (BSE), can be written as follows [51]:

$$
\begin{aligned}
L\left(1,2 ; 1^{\prime}, 2^{\prime}\right)= & L_{0}\left(1,2 ; 1^{\prime}, 2^{\prime}\right)+\int d(3456) L_{0}\left(1,4 ; 1^{\prime} 3\right) \\
& \times K(3,5 ; 4,6) L\left(6,2 ; 5,2^{\prime}\right)
\end{aligned}
$$

where the variables (1), etc. combine position, spin, and time coordinates of an electron or hole. $L_{0}\left(1,2 ; 1^{\prime}, 2^{\prime}\right)=G_{1}\left(1,2^{\prime}\right) G_{1}\left(2,1^{\prime}\right)$ corresponds to the free propagation of an electron and hole without interaction. $L\left(1,2 ; 1^{\prime}, 2^{\prime}\right)$ describes the electron-hole correlation function, which is affected by the electron-hole interaction kernel $K(3,5 ; 4,6) . K(3,5 ; 4,6)$ itself is connected to an electron-hole exchange contribution $v$ and the electron-hole attraction in form of the screened Coulomb interaction $W[51]$ :

$$
K(3,5 ; 4,6)=-i \delta(3,4) \delta\left(5^{-}, 6\right) v(3,6)+i \delta(3,6) \delta(4,5) W\left(3^{+}, 4\right)
$$

To solve equation (18) explicitly, basis functions, e.g. Bloch functions are used. By doing so, the problem breaks down to a generalized eigenvalue problem [52] of the form

$$
\boldsymbol{L}=\left(\boldsymbol{H}^{2 p}-\boldsymbol{I} \omega\right)^{-1} \boldsymbol{F}
$$

with $\boldsymbol{H}^{2 p}$ being an effective two-particle Hamiltonian, $\boldsymbol{I}$ the identity operator and $\boldsymbol{F}$ the matrix of Fermi-Dirac occupation numbers.

Optical excitation spectra can be obtained evaluating the macroscopic dielectric function $\varepsilon_{M}[52]$ :

$$
\varepsilon_{M}(\omega)=1+\lim _{\boldsymbol{q} \rightarrow 0} v_{0}(\boldsymbol{q}) \times \sum_{\lambda} \frac{\left|\sum_{\nu, c ; \boldsymbol{k}}\left\langle v, \boldsymbol{k}\left|e^{-i \boldsymbol{q} \boldsymbol{r}}\right| c, \boldsymbol{k}\right\rangle A_{\lambda}^{(v, c ; \boldsymbol{k})}\right|^{2}}{\left(E_{\lambda}-\omega\right)}
$$

$v$ and $c$ denote occupied and unoccupied electron states, respectively. $A_{\lambda}$ are the coefficients of the states $|\lambda\rangle$ in the chosen basis. The optical excitation spectrum is directly connected to the imaginary part of $\varepsilon_{M}$, often denoted as $\varepsilon_{2}$. Results for the optical spectra of $M \mathrm{Fe}_{2} \mathrm{O}_{4}$ can be found in Section 3.3.1. Since GW/BSE calculations are based on a single-determinant broken-symmetry DFT wavefunction, they cannot describe inraatomic $d-d$ transitions. This has to be taken into account in comparisons between calculated and measured optical spectra. 


\subsubsection{Dynamical mean field theory}

With dynamical mean field theory (DMFT) materials with strongly correlated electrons can be treated non-perturbatively. The main idea is to approximate a manybody lattice problem by a single-site effective problem with less degrees of freedom. In the Hubbard model, the on-site interaction between electrons of opposite spin is described by the parameter $U[53,54]$ :

$$
H_{H u b}=\sum_{\langle i j\rangle \sigma} t_{i j}\left(c_{i \sigma}^{\dagger} c_{j \sigma}+c_{j \sigma}^{\dagger} c_{i \sigma}\right)+U \sum_{i} n_{i \uparrow} n_{i \downarrow} .
$$

$c_{i \sigma}^{\dagger}\left(c_{i \sigma}\right)$ are creation (annihilation) operators for fermions with spin $\sigma$ and position $i, t_{i j}$ the hopping strength from site $i$ to site $j$, and $n_{i \sigma}=c_{i \sigma}^{\dagger} c_{i \sigma}$.

In practice, the lattice problem can be mapped onto a self-consistent singleimpurity Anderson model. One lattice site is removed from the rest of the lattice, which now contains a cavity (the impurity). The remaining lattice is replaced by a particle bath, the dynamical mean field. Via hybridization, the bath is coupled to the cavity. This approach can be represented by the Anderson Impurity Hamiltonian [54],

$$
H_{A I M}=\underbrace{\sum_{l \sigma} \tilde{\epsilon} a_{l \sigma}^{\dagger} a_{l \sigma}}_{H_{\text {bath }}}+\underbrace{\sum_{l \sigma} V_{l}\left(a_{l \sigma}^{\dagger} c_{o \sigma}+c_{o \sigma}^{\dagger} a_{l \sigma}\right)}_{H_{\text {hybr }}}-\underbrace{\mu \sum_{\sigma} c_{o \sigma}^{\dagger} c_{o \sigma}+U n_{o \uparrow} n_{o \downarrow}}_{H_{l o c}},
$$

where $H_{b a t h}$ describes the electronic levels $\tilde{\epsilon}_{l}$ of the bath, $H_{l o c}$ describes the impurity and $H_{h y b r}$ describes the hybridization featuring the hybridization term $V_{l}$. $a_{l \sigma}^{\dagger}\left(a_{l \sigma}\right)$ correspond to creation (annihilation) operator of the impurity. The problem can now be solved by self-consistent determination of the degrees of freedom of the bath, represented by an appropriate hybridization function [55]. To the best of the authors' knowledge, DMFT has not been applied to ferrites, yet.

\subsection{Calculation of IR/Raman spectra under periodic boundary conditions}

In standard quantum-chemical calculations of normal modes, the BornOppenheimer potential energy surface in its harmonic approximation $V(0)$ is used [56]:

$$
V(0)=\frac{1}{2} \sum_{i j} u_{i} \mathcal{H}_{i j} u_{j}
$$


$u_{i}$ is the displacement from equilibrium, and $\mathcal{H}$ the Hessian matrix evaluated at equilibrium:

$$
\mathcal{H}_{i j}=\frac{1}{2}\left[\frac{\partial^{2} V(\boldsymbol{r})}{\partial u_{i} \partial u_{j}}\right]_{0} .
$$

$W$ is the mass weighted Hessian:

$$
W_{i j}=\frac{\mathcal{H}_{i j}}{\sqrt{M_{i} M_{j}}} .
$$

To calculate vibrational spectra, $W$ has to be diagonalized to find a set of eigenvalues $\kappa_{j}$.

By introducing periodicity, the coordinates $q_{i}$ become dependent on the k-points:

$$
q_{i}(\boldsymbol{k})=N \sum_{\boldsymbol{G}} e^{-i \boldsymbol{k} \cdot \boldsymbol{G}} q_{i}^{\boldsymbol{G}} .
$$

This introduces a block-form of $W$ where the $\boldsymbol{k}^{\text {th }}$ block of the factorized $W$ matrix has the form

$$
W_{i j}(\boldsymbol{k})=\sum_{\boldsymbol{G}} e^{i \boldsymbol{k} \cdot \boldsymbol{G}} \frac{\mathcal{H}_{i j}^{\mathbf{O G}}}{\sqrt{M_{i} M_{j}}} .
$$

$\mathcal{H}_{i j}^{O G}$ is the second derivative of $V(\boldsymbol{r})$ at equilibrium, having atom $i$ in the original cell $\mathbf{0}$ and atom $j$ in a translated cell $\boldsymbol{G}$. In practice, only the $\Gamma=(0,0,0)$ point is considered, reducing equation (28) to

$$
W_{i j}(\mathbf{0})=\sum_{\boldsymbol{G}} \frac{\mathcal{H}_{i j}^{\mathbf{0 G}}}{\sqrt{\bar{M}_{i} M_{j}}} .
$$

The calculation of IR intensities [57] requires evaluation of the derivative of the dipole moment $\mu$ with respect to the atomic positions $\boldsymbol{r}=\left(r_{a}, r_{b}, r_{c}\right)$ :

$$
\frac{\partial \mu_{b}}{\partial r_{a}^{A}}=\left.\frac{\partial^{2} E_{t o t}}{\partial r_{a}^{A} \partial \epsilon_{b}}\right|_{\boldsymbol{\epsilon}=0, r_{0}}
$$

$r_{a}^{A}$ denotes the Cartesian coordinate of atom $A$ along direction $a$ and $\boldsymbol{\epsilon}=$ $\left(\epsilon_{a}, \epsilon_{b}, \epsilon_{c}\right)$ is the electric field. Raman intensities [58] are evaluated as the partial derivatives of the polarizability tensor $\boldsymbol{\alpha}$ with respect to the atomic positions:

$$
\left.\frac{\partial \alpha_{b, c}}{\partial r_{a}^{A}}\right|_{r=0}=\left.\frac{\partial^{3} E_{t o t}}{\partial r_{a}^{A} \partial \epsilon_{b} \partial \epsilon_{c}}\right|_{\boldsymbol{\epsilon}=0, r_{0}}
$$


The evaluation of equations (30) and (31) for periodic systems is not straightforward. One approach is to reformulate the electronic interaction operator $\hat{\Omega}$ [58] to

$$
\hat{\Omega}(\boldsymbol{k})=\sum_{b} \epsilon_{b} \hat{\Omega}_{b}(\boldsymbol{k})
$$

and to treat it perturbatively as in the Coupled-perturbed Hartree-Fock (CPHF) or Kohn-Sham (CPKS) approach (cf. Section 2.1.3). This treatment avoids the calculation of gradients of the wavefunction and instead uses second derivatives to the electric fields, which can be determined analytically.

\subsection{Construction of surface models}

While theoretical calculations of infinite crystals provide detailed insight of the bulk properties, real crystals are finite systems terminated by two-dimensional surfaces. Many chemical processes of interest like heterogeneous catalysis take place at the interface between a crystal's surface and the environment. Gaining insight on the process of photocatalytic water splitting on ferrites as PECs thus means investigating ferrite surfaces. The surface models considered in this work are cut from the crystalline bulk via their Miller indices and are periodic in two dimensions but finite in the third [59]. A sound surface model that will yield physically correct results for surface properties of interest should be stoichiometric, symmetric and resultingly free of dipoles parallel to the surface normal. Yet, as Tasker explains, not every surface of an ionic crystal, such as a ferrite, can fulfill these criteria [60]. Given that this a consequence of surface geometry, Tasker classifies them accordingly as two non-polar and one polar surface type. Tasker type 1 surfaces are inherently non-polar and type 2 surfaces can be set up in nonpolar ways, whereas type 3 surfaces are always polar. Low index spinel surfaces are Tasker type 3 surfaces. Their atomic layers are occupied by different numbers of metal cations and oxide anions, resulting in layers of different charge which can not be stacked in a way that results in a stoichiometric and symmetric surface model. The total number of layers of any type of surface model has to be increased to mimic bulk-like behavior in the center of the slab. For nonpolar surface models, this ensures convergence of the properties of interest, for example, the surface energy or band positions. However, for unmodified polar Tasker type 3 surfaces, energies will diverge with increasing number of layers. Despite this, polar surface terminations exist, but as Tasker mentions and as discussed thoroughly in literature, they are stabilized via (extensive) reconstruction, via defect formation, through adsorption or other processes altering their structure [61, 62]. 
Structural modifications considered in this work are described in the following. Defective surface models are constructed by removing one or several atoms from the topmost and subsequently the bottom layer of a symmetric surface model to achieve stoichiometry. In some cases the second layer from top and bottom have to be partially depleted as well. Results for defective surface models are presented in the first part of Section 3.4.1. In the majority of cases surfaces are exposed to the environment and are in contact with it which can lead to the adsorption of foreign species such as water oder hydroxyl species. In the case of Tasker type 3 surfaces, the formal addition of a species can lead to a symmetric model that represents the stoichiometry of the material of interest and the adsorbed species. Surface models with adsorbed species are discussed in the second part of Section 3.4.1. Moreover, a unique feature of the CRYSTAL code allows the investigation of stoichiometric polar surfaces without removing or adding atoms or employing a dipole correction. Any two-dimensional surface can be rolled up into a nanotube [63]. Its circular symmetry leads to the cancellation of the inherent dipole moment of polar surfaces and allows the investigation of different terminations within one model. One would expect the strain on the surface caused by the nanotube's curvature to affect the electronic structure, yet previous work has shown that the electronic structure of one-dimensional nanotubes and their two-dimensional counterparts are quite similar [64]. Nanotube models are discussed in the last part of Section 3.4.1.

\section{Examples of application}

\subsection{Electronic properties of ferrites}

\subsubsection{Theoretical results}

Table 1 shows theoretical results for the electronic band gap of $M \mathrm{Fe}_{2} \mathrm{O}_{4}$ with $M=$ $\mathrm{Mn}, \mathrm{Fe}, \mathrm{Co}, \mathrm{Ni}, \mathrm{Cu}, \mathrm{Zn}$ as found in literature. As can be seen, most of the calculations were carried out using $\mathrm{GGA}+\mathrm{U}$. Since the parameter $\mathrm{U}$ is usually adjusted to reproduce a certain property of the material, the resulting band gaps vary considerably. Furthermore, many of the references provided in Table 1 are not providing exact specification of the magnetic structure. The band gaps calculated with hybrid DFT for $\mathrm{Fe}_{3} \mathrm{O}_{4}$ and $\mathrm{NiFe}_{2} \mathrm{O}_{4}$ exceed those that are calculated with GGA+U in both cases. As pointed out in Section 2.1.3, standard hybrid DFT is not parameterfree, the electronic properties are dependent on the amount of Fock exchange. Standard hybrid DFT functionals such as HSE06 tend to overestimate the electronic band gaps of transition metal oxides. The only theoretical study using the 
Tab. 1: Calculated fundamental band gaps of $\mathrm{MFe}_{2} \mathrm{O}_{4}(M=\mathrm{Mn}, \mathrm{Fe}, \mathrm{Co}, \mathrm{Ni}, \mathrm{Cu}, \mathrm{Zn})$ as found in literature.

\begin{tabular}{|c|c|c|c|}
\hline$M$ & $E_{g}^{c a l c}[\mathrm{eV}]$ & Method & Reference \\
\hline \multirow[t]{2}{*}{$\mathrm{Mn}$} & 0.4 & $\mathrm{GGA}+\mathrm{U}$ & [19] \\
\hline & 0.87 & $\mathrm{GGA}+\mathrm{U}$ & [12] \\
\hline \multirow[t]{9}{*}{$\mathrm{Fe}$} & 0.0 & $\mathrm{GGA}+\mathrm{U}$ & [6] \\
\hline & $0.00-0.28$ & $\mathrm{GGA}+\mathrm{U}$ & [5] \\
\hline & $0.00-0.53$ & $\mathrm{GGA}+\mathrm{U}$ & [24] \\
\hline & 0.19 & LSDA+U & [3] \\
\hline & 0.500 & $\mathrm{GGA}+\mathrm{U}$ & [14] \\
\hline & 0.72 & $\mathrm{GGA}+\mathrm{U}$ & [10] \\
\hline & 0.75 & $\mathrm{GGA}+\mathrm{U}$ & [18] \\
\hline & 2.1 & $\mathrm{GGA}+\mathrm{U}$ & [23] \\
\hline & 2.2 & hybrid $\mathrm{DFT}^{\mathrm{a}}$ & [23] \\
\hline \multirow[t]{5}{*}{ Co } & 0.09 & $\mathrm{GGA}+\mathrm{U}$ & [10] \\
\hline & 0.63 & $\mathrm{LSDA}+\mathrm{U}$ & [3] \\
\hline & $0.75-1.41$ & $\mathrm{GGA}+\mathrm{U}$ & [24] \\
\hline & 0.9 & $\mathrm{GGA}+\mathrm{U}$ & [9] \\
\hline & 1.171 & $\mathrm{GGA}+\mathrm{U}$ & [65] \\
\hline \multirow[t]{6}{*}{$\mathrm{Ni}$} & 0.97 & $\mathrm{GGA}+\mathrm{U}$ & [9] \\
\hline & 0.99 & $\mathrm{LSDA}+\mathrm{U}$ & [3] \\
\hline & 1.6 & $\mathrm{LSDA}+\mathrm{U}$ & [15] \\
\hline & 1.63 & $\mathrm{GGA}+\mathrm{U}$ & [18] \\
\hline & 1.675 & $\mathrm{GGA}+\mathrm{U}$ & [65] \\
\hline & 2.7 & hybrid DFT ${ }^{b}$ & [15] \\
\hline $\mathrm{Cu}$ & 0.23 & $\mathrm{GGA}+\mathrm{U}$ & [8] \\
\hline \multirow[t]{3}{*}{$\mathrm{Zn}$} & 1.68 & $\mathrm{GGA}+\mathrm{U}$ & [18] \\
\hline & 1.821 & $\mathrm{GGA}+\mathrm{U}$ & [65] \\
\hline & 2.02 & $\mathrm{G}_{0} \mathrm{~W}_{0}$ & [33] \\
\hline
\end{tabular}

${ }^{\mathrm{a}} \mathrm{HSE}, 15 \%$ Fock exchange, ${ }^{\mathrm{b}} \mathrm{HSE} 06$.

$G W$ approach is for $\mathrm{ZnFe}_{2} \mathrm{O}_{4}$ [33]. The resulting band gap of $2.02 \mathrm{eV}$ can be viewed as the most accurate result, but as evaluated in Section 2.1.4, it is still dependent on the underlying ground state calculation, as well as the variant of $G W$.

As will be pointed out in Section 3.1.2, the experimental literature results for band gaps of $\mathrm{MFe}_{2} \mathrm{O}_{4}$ show a high variation, too. An explanation for this high variation could not be obtained so far, even by considering the theoretical predictions from Table 1.

In a recent study [66] we investigated the variation of electronic properties due to ion distribution and spin state in order to provide a tentative explanation for the 
observed scattering of experimental results. Cation inversion and the magnetic ordering of $\mathrm{MFe}_{2} \mathrm{O}_{4}$ are crucial parameters that influence the electronic structure. Theoretical calculations allow to investigate these effects, since the cation distribution and magnetic structure can be modeled explicitly.

Every cation distribution and spin state has to be treated as different material with different properties. That is why we decided to employ dielectric dependent self-consistent hybrid (DDSCH) functionals as they are computationally more efficient than $G W$ methods. Within this approach, every configuration is treated with an optimal exchange fraction.

The investigated configurations are shown in Table 2. The abbreviation for the final configuration composes of the capital letter $A-G$ for the cation distribution and the abbreviation for the spin configuration, where ' $\mathrm{fm}$ ' indicates ferromagnetism, 'afm' antiferromagnetism and 'fi' ferrimagnetism. All calculations use the broken symmetry approach. Details on the generation of the configurations can be found in reference [66].

Tab. 2: Fractional coordinates of tetrahedral and octahedral positions in the primitive unit cell and their possible occupancies.

\begin{tabular}{|c|c|c|c|c|c|c|}
\hline \multirow[t]{2}{*}{ Position } & \multicolumn{2}{|r|}{ Tet (8a) } & \multicolumn{4}{|c|}{$\overline{\text { Oct (16d) }}$} \\
\hline & 1 & 2 & 3 & 4 & 5 & 6 \\
\hline$x$ & 0.125 & -0.125 & -0.5 & -0.5 & 0.0 & -0.5 \\
\hline y & 0.125 & -0.125 & -0.5 & -0.5 & -0.5 & 0.0 \\
\hline$z$ & 0.125 & -0.125 & -0.5 & 0.0 & -0.5 & -0.5 \\
\hline Variant & \multicolumn{6}{|c|}{ Occupancy } \\
\hline $\bar{A}$ & $M^{\prime \prime}$ & $M^{\prime \prime}$ & $\mathrm{Fe}^{\mathrm{III}}$ & $\mathrm{Fe}^{\mathrm{III}}$ & $\mathrm{Fe}^{I I I}$ & $\mathrm{Fe}^{\mathrm{III}}$ \\
\hline B & $\mathrm{Fe}^{\mathrm{III}}$ & $\mathrm{Fe}^{I I I}$ & $M^{\prime \prime}$ & $M^{\prime \prime}$ & $\mathrm{Fe}^{\prime I I}$ & $\mathrm{Fe}^{\prime I I I}$ \\
\hline C & $M^{\prime \prime \prime}$ & $M^{\prime \prime \prime}$ & $\mathrm{Fe}^{\prime \prime}$ & $\mathrm{Fe}^{\| l}$ & $\mathrm{Fe}^{\prime I I I}$ & $\mathrm{Fe}^{I I I I}$ \\
\hline D & $\mathrm{Fe}^{\prime \prime}$ & $\mathrm{Fe}^{\prime \prime}$ & $\mathrm{Fe}^{\mathrm{III}}$ & $\mathrm{Fe}^{\mathrm{III}}$ & $M^{\prime \prime \prime}$ & $M^{\prime \prime \prime}$ \\
\hline $\mathrm{E}$ & $\mathrm{Fe}^{\prime I I}$ & $\mathrm{Fe}^{\mathrm{III}}$ & $\mathrm{Fe}^{\prime \prime}$ & $\mathrm{Fe}^{\| \prime}$ & $M^{\prime \prime \prime}$ & $M^{I I I}$ \\
\hline $\mathrm{F}$ & $M^{\mathrm{IV}}$ & $M^{\mathrm{IV}}$ & $\mathrm{Fe}^{\prime \prime}$ & $\mathrm{Fe}^{\prime \prime}$ & $\mathrm{Fe}^{\prime \prime}$ & $\mathrm{Fe}^{\prime \prime}$ \\
\hline G & $\mathrm{Fe}^{\prime \prime}$ & $\mathrm{Fe}^{l l}$ & $\mathrm{Fe}^{\prime l}$ & $\mathrm{Fe}^{\prime \prime}$ & $M^{\mathrm{IV}}$ & $M^{\mathrm{IV}}$ \\
\hline $\mathrm{fm}$ & $\uparrow$ & $\uparrow$ & $\uparrow$ & $\uparrow$ & $\uparrow$ & $\uparrow$ \\
\hline afm1 & $\uparrow$ & $\downarrow$ & $\uparrow$ & $\uparrow$ & $\downarrow$ & $\downarrow$ \\
\hline $\operatorname{afm} 2$ & $\uparrow$ & $\downarrow$ & $\uparrow$ & $\downarrow$ & $\uparrow$ & $\downarrow$ \\
\hline fi1 & $\downarrow$ & $\downarrow$ & $\uparrow$ & $\uparrow$ & $\uparrow$ & $\uparrow$ \\
\hline fi2 & $\uparrow$ & $\uparrow$ & $\downarrow$ & $\downarrow$ & $\uparrow$ & $\uparrow$ \\
\hline fi3 & $\uparrow$ & $\uparrow$ & $\uparrow$ & $\uparrow$ & $\downarrow$ & $\downarrow$ \\
\hline fi4 & $\uparrow$ & $\uparrow$ & $\uparrow$ & $\downarrow$ & $\downarrow$ & $\downarrow$ \\
\hline fi5 & $\uparrow$ & $\uparrow$ & $\downarrow$ & $\downarrow$ & $\uparrow$ & $\downarrow$ \\
\hline
\end{tabular}


Before employing dielectric-dependent self-consistent hybrid (DDSCH) functionals, we optimized the structures of the generated conformations. This was done by using efficient ECP basis sets and the PW1PW functional.

The relaxed structures were used for the DDSCH procedure. To increase accuracy, the recently developed rev2-POB-TZVP basis sets [67] were used for the DDSCH calculations. Details on the settings can be found in reference [66].

Table 3 shows the results for the static dielectric constant and electronic band gap for the most important configurations, selected in an energy range of $20 \mathrm{~kJ} / \mathrm{mol}$ around the most stable one. Because of excitonic effects, the energy of excited states is generally smaller than the electronic gap, and thus the optical gap is usually smaller than the fundamental gap. Exceptions can occur due to selection rules. Since the experimentally determined band gaps are from optical measurements, and the DDSCH results are fundamental band gaps, we expect an energy difference in the order of the excitonic binding energy of transition metals, which can be in the range of $0.3-0.6 \mathrm{eV}$ [68].

The results for $\mathrm{MgFe}_{2} \mathrm{O}_{4}$ and $\mathrm{ZnFe}_{2} \mathrm{O}_{4}$ are comparable, since the electron configurations of $d^{0}$ for $\mathrm{Mg}^{2+}$ and $d^{10}$ for $\mathrm{Zn}^{2+}$ prohibit an involvement of these elements to the magnetic structure. For both of them the DDSCH results overestimate experimental results or are in their upper range. The DDSCH results for all of the low-energy configurations of $\mathrm{MnFe}_{2} \mathrm{O}_{4}, \mathrm{CoFe}_{2} \mathrm{O}_{4}$, and $\mathrm{NiFe}_{2} \mathrm{O}_{4}$ lie within the experimental range for the band gap. For $\mathrm{Fe}_{3} \mathrm{O}_{4}$, most of the generated configurations have a conducting ground state. This is in line with previous theoretical findings (cf. Table 1). A band gap of $0.91 \mathrm{eV}$ was determined for the inverse, ferrimagnetic configuration B-fi4, which is in the range of experimental results. The calculated band gaps for both configurations of $\mathrm{CuFe}_{2} \mathrm{O}_{4}$ are smaller than the experimental results. However, since the SCF stability for $\mathrm{CuFe}_{2} \mathrm{O}_{4}$ was not satisfactory, these results have to be taken with care.

For $M=\mathrm{Sc}$, Ti, V, Cr, no experimental band gaps are available in literature. Regarding the size of the band gap listed in Table 3, only $\mathrm{ScFe}_{2} \mathrm{O}_{4}$ and $\mathrm{CrFe}_{2} \mathrm{O}_{4}$ are possible candidates as electrode material for photoelectrochemical water splitting. For a detailed discussion of the results from Table 3, see reference [66].

As can be seen from these results the flexibility of $M \mathrm{Fe}_{2} \mathrm{O}_{4}$ regarding cation distribution and magnetic structure offers an explanation for the high variety of experimental results for most of the compounds. However, this is not true for $\mathrm{MgFe}_{2} \mathrm{O}_{4}$ and $\mathrm{ZnFe}_{2} \mathrm{O}_{4}$, where all of the calculated results are in the range of $\sim 3 \mathrm{eV}$. Experiments of Sultan et al. suggest a strong dependency of the band gap energy of $\mathrm{ZnFe}_{2} \mathrm{O}_{4}$ on the oxygen partial pressure [100]. Hence, the small band gap of $\sim 2 \mathrm{eV}$ obtained in many experiments might be induced by oxygen vacancies. 
Tab. 3: Energetics, dielectric constant, and electronic band gap of the most stable spin configurations for $M=M g$, Sc-Zn calculated with DDSCH functionals [66].

\begin{tabular}{|c|c|c|c|c|c|c|}
\hline $\bar{M}$ & State & $\Delta E[\mathrm{~kJ} / \mathrm{mol}]$ & $\varepsilon$ & $\varepsilon_{o p t}^{e x p}$ & $\mathrm{E}_{g}[\mathrm{eV}]$ & $\mathrm{E}_{g}^{\text {exp }}[\mathrm{eV}]$ \\
\hline \multirow[t]{3}{*}{$\mathrm{Mg}$} & B-fi4 & 0.0 & 4.96 & & 3.57 & $1.85-2.43^{\mathrm{a}}$ \\
\hline & A-fm & 18.5 & 4.22 & & 3.56 & \\
\hline & B-afm2 & 19.7 & 4.76 & & 3.45 & \\
\hline \multirow[t]{3}{*}{$\mathrm{Sc}$} & E-fi2 & 0.0 & 5.06 & & 2.77 & \\
\hline & D-fi1 & 7.6 & 4.83 & & 2.81 & \\
\hline & D-afm2 & 15.4 & 5.07 & & 1.85 & \\
\hline \multirow[t]{2}{*}{$\mathrm{Ti}$} & F-afm1 & 0.0 & 4.44 & & 3.18 & \\
\hline & $\mathrm{G}-\mathrm{fm}$ & 16.2 & 5.48 & & 0.00 & \\
\hline V & D-fi2 & 0.0 & - & & 0.00 & \\
\hline \multirow[t]{3}{*}{$\mathrm{Cr}$} & D-fi1 & 0.0 & 5.52 & & 2.25 & \\
\hline & D-fi2 & 0.7 & 5.57 & & 2.34 & \\
\hline & D-fi3 & 10.5 & - & & 0.00 & \\
\hline $\mathrm{Mn}$ & A-fi1 & 0.0 & 6.48 & $\left(4.24^{b}, 4.84^{b}\right)$ & 1.07 & $0.98-2.37^{c}$ \\
\hline \multirow[t]{4}{*}{$\mathrm{Fe}$} & B-fi4 & 0.0 & 10.94 & & 0.91 & $0.12-1.92^{\mathrm{d}}$ \\
\hline & A-fi2 & 2.8 & - & & 0.00 & \\
\hline & B-fi1 & 5.0 & - & & 0.00 & \\
\hline & B-fi2 & 15.3 & 7.88 & & 0.00 & \\
\hline \multirow[t]{4}{*}{ Co } & B-fi1 & 0.0 & 6.76 & $6.66^{\mathrm{e}}$ & 1.28 & $1.17-2.62^{f}$ \\
\hline & A-fi1 & 4.1 & 5.71 & & 2.06 & \\
\hline & B-fi4 & 13.4 & 6.71 & & 1.54 & \\
\hline & A-afm1 & 19.8 & 5.85 & & 2.02 & \\
\hline \multirow[t]{2}{*}{$\mathrm{Ni}$} & B-fi1 & 0.0 & 6.42 & $5.52^{\mathrm{e}}$ & 2.27 & $1.52-3.54^{\mathrm{g}}$ \\
\hline & B-fi4 & 12.2 & 6.34 & & 1.82 & \\
\hline \multirow[t]{2}{*}{$\mathrm{Cu}$} & B-fi1 & 0.0 & 7.37 & $\left(4.37^{b}, 5.15^{b}\right)$ & 0.95 & $1.37-1.61^{h}$ \\
\hline & B-fi3 & 18.9 & 6.72 & & 1.06 & \\
\hline \multirow[t]{3}{*}{$\mathrm{Zn}$} & $A-f m$ & 0.0 & 4.74 & $5.76^{\mathrm{e}}$ & 3.00 & $1.78-3.25^{\mathrm{i}}$ \\
\hline & A-afm1 & 2.2 & 4.89 & & 2.89 & \\
\hline & B-fi4 & 14.0 & 5.57 & & 2.71 & \\
\hline
\end{tabular}

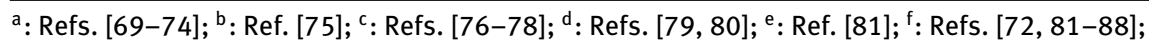

${ }^{\mathrm{g}}$ : Refs. $[15,81,83,85,87,89-95]$; $^{\text {h }}$ : Refs. [84, 85, 91, 96, 97]; ': Refs. [81, 84, 85, 98-101]

To test this hypothesis, we generated models for oxygen-deficient $\mathrm{ZnFe}_{2} \mathrm{O}_{4}$. We used configuration A-afm1 as starting point, despite A-fm being the most stable one according to our results, since $\mathrm{ZnFe}_{2} \mathrm{O}_{4}$ is presumed to be an aniferromagnet. For the generation of the oxygen-deficient models we removed one oxygen atom 
from the conventional unit cell, which leads to a vacancy concentration of 3.125\%. To increase the variational freedom of the wavefunction in the defective region, only the nuclear charge and shell electron charges were removed, and the basis set was left centered at the atomic position.

The structure of the defect models was again optimized with the settings from reference [66]. After relaxation, a single point calculation with the optimal fock exchange obtained for pristine $\mathrm{ZnFe}_{2} \mathrm{O}_{4}$ configuration A-afm1 was performed employing the rev2-pob-TZVP basis sets. The band gap range of the 12 defective models in comparison to pristine $\mathrm{ZnFe}_{2} \mathrm{O}_{4}$ can be found in Table 4. For most of the models, the spin-up and spin-down band gaps are no longer equal, due to the reduced symmetry.

The results for the oxygen-deficient models shown in Table 4 show a clear band gap narrowing in comparison to pristine $\mathrm{ZnFe}_{2} \mathrm{O}_{4}$. This observation serves as an explanation for the two different sets of experimental band gaps for $\mathrm{ZnFe}_{2} \mathrm{O}_{4}$. If the narrow band gap of $\sim 2 \mathrm{eV}$ is due to oxygen defects, the applicability of $\mathrm{ZnFe}_{2} \mathrm{O}_{4}$ as a photocatalyst is limited. The presence of oxygen defects will play a crucial role for the performance of the material, since the charge carrier mobility might be reduced.

To further investigate the nature of electronic transitions in the case of $\mathrm{ZnFe}_{2} \mathrm{O}_{4}$, the highest occupied crystal orbital (HOCO), and the lowest unoccupied crystal orbital (LUCO) were inspected. Figure 1 shows the spin-up HOCO and spinup LUCO of pristine $\mathrm{ZnFe}_{2} \mathrm{O}_{4}$ in configuration A-afm1. The HOCO mainly consists of oxygen $2 p$ orbitals. The irregular shape shows that a hybridization with iron $3 \mathrm{~d}$ orbitals is present. The LUCO consists of Fe $3 \mathrm{~d}$ orbitals, which is expected for an iron oxide. Figure 2 shows the spin-up HOCO and spin-up LUCO of the same configuration of $\mathrm{ZnFe}_{2} \mathrm{O}_{4}$ with $3.125 \%$ oxygen vacancies. The $\mathrm{HOCO}$ now consists of one single $\mathrm{Fe} 3 \mathrm{~d}$ orbital close to the oxygen vacancy indicating that these $\mathrm{Fe}^{3+}$ ions were reduced to $\mathrm{Fe}^{2+}$. A corresponding effect could already be observed for $\mathrm{TiO}_{2}$ with oxygen defects [102]. This orbital can act as a recombination center. The LUCO of the defective model consists of strings of Fe 3d orbitals, which might enhance the conductivity in this direction.

Tab. 4: Electronic band gaps of antiferromagnetic $\mathrm{ZnFe}_{2} \mathrm{O}_{4}$ with an oxygen vacancy concentration of $3.125 \%$ in comparison to pristine $\mathrm{ZnFe}_{2} \mathrm{O}_{4} \mathrm{~A}$-afm1, calculated with a DDSCH approach.

\begin{tabular}{lrr}
\hline Model & $E_{g}^{\text {spin-up }}[\mathrm{eV}]$ & $E_{g}^{\text {spin-down }}[\mathrm{eV}]$ \\
\hline Pristine & 2.89 & 2.89 \\
Defective & $1.53-1.79$ & $1.53-1.80$ \\
\hline
\end{tabular}



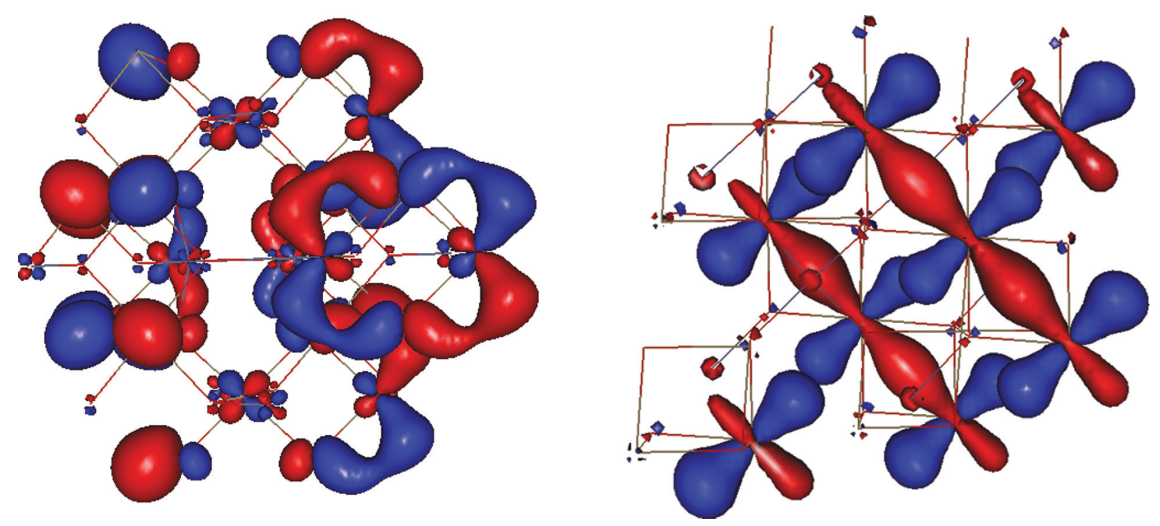

Fig. 1: Spin-up HOCO (left) and spin-up LUCO (right) of pristine $\mathrm{ZnFe}_{2} \mathrm{O}_{4}$, configuration A-afm1, displayed in the conventional unit cell.
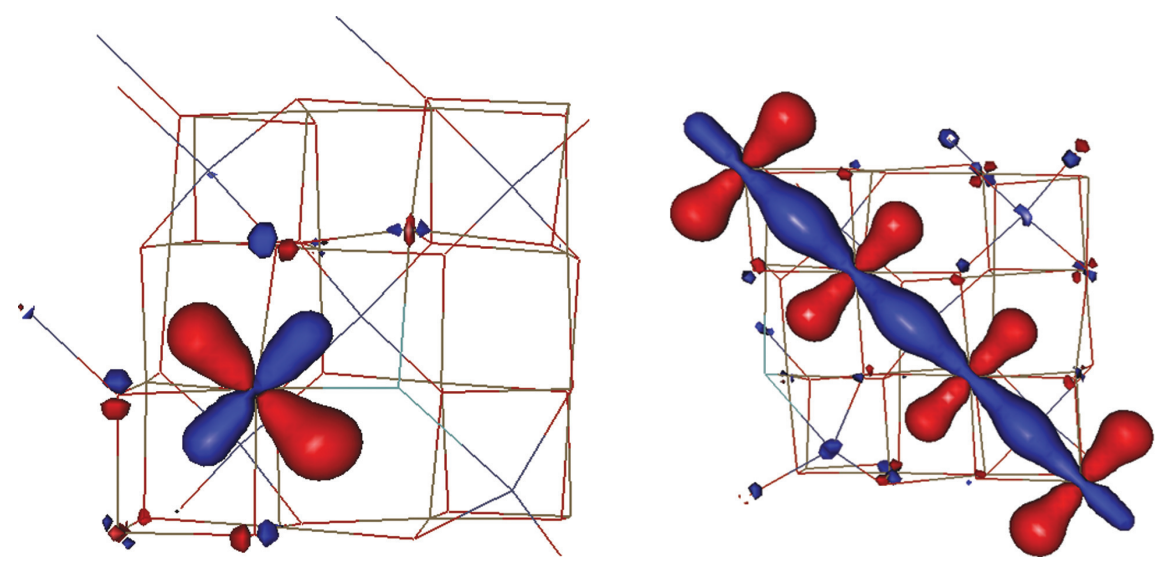

Fig. 2: Spin-up HOCO (left) and spin-up LUCO (right) of $\mathrm{ZnFe}_{2} \mathrm{O}_{4}$ configuration A-afm1 with $3.125 \%$ oxygen defects, displayed in the conventional unit cell.

\subsubsection{Experimental literature results}

It is well known that the dielectric properties of ferrites depend on the grain size of the sample $[103,104]$. Therefore, a comparison between values of dielectric constants predicted by theoretical calculations and achieved experimentally is not straightforward. However, it is meaningful to compare the experimentally observed and calculated trends concerning the impact of the degree of inversion on the dielectric properties. In this regard, reports in which the effect of the degree of inversion on the dielectric properties is discussed are hardly found in 
the scientific literature. For $\mathrm{ZnFe}_{2} \mathrm{O}_{4}$, it was shown that samples similar in particle size, crystallite size, and crystallinity exhibit an increase in the electrical conductivity and a decrease in dielectric constant as the degree of inversion increases [105]. This experimental observation contradicts the theoretical calculations presented in Table 3, in which the dielectric constant of inverse $\mathrm{ZnFe}_{2} \mathrm{O}_{4}$ is higher than the dielectric constant of normal $\mathrm{ZnFe}_{2} \mathrm{O}_{4}$. However, it should be taken into account that the calculations for the normal and inverse structures were done considering a ferromagnetic (fm)/antiferromagnetic (afm) and ferrimagnetic (fi) spin configuration, respectively. The spin configuration of the $\mathrm{ZnFe}_{2} \mathrm{O}_{4}$ samples analyzed experimentally is unknown.

The optical band gap of ferrites is an important parameter in the field of photoelectrochemistry. A large variety of experimental results for the optical band gaps of $M \mathrm{Fe}_{2} \mathrm{O}_{4}$ can be found (see Table 5). The band gaps are mostly determined using UV-Vis diffuse reflectance measurement, or UV-Vis absorption spectroscopy. As can be seen from Table 5, the determined band gap depends on the particle shape and size. The general observation is, that with a smaller particle size, the band gap increases. Interestingly, this is also true for particle sizes that are too big to be affected by the quantum size effect, which was shown not to apply to particles that are larger than $2.1 \mathrm{~nm}$ in the case of $\mathrm{TiO}_{2}$ [106]. However, there are also exceptions to this observation.

The measured optical band gaps for $\mathrm{CoFe}_{2} \mathrm{O}_{4}$ and $\mathrm{ZnFe}_{2} \mathrm{O}_{4}$ can be divided in two groups, 1.17-1.58 eV and 2.44-2.62 eV for $\mathrm{CoFe}_{2} \mathrm{O}_{4}$, and 1.78-2.01 eV and 2.61-3.25 eV for $\mathrm{ZnFe}_{2} \mathrm{O}_{4}$. While for $\mathrm{CoFe}_{2} \mathrm{O}_{4}$ these values most probably belong to indirect and direct transitions [82], this explanation does not hold for $\mathrm{ZnFe}_{2} \mathrm{O}_{4}$, where indirect and direct transition differ by only $\sim 0.1 \mathrm{eV}$ [98]; see also our own measurements in Section 3.3.2. As mentioned in Section 3.1.1, a possible explanation for this observation for $\mathrm{ZnFe}_{2} \mathrm{O}_{4}$ was given by Sultan and Singh [100] whose results show an increase of the band gap with enhanced oxygen pressure. Thus, oxygen deficiency might induce a reduction of the band gap energy. Zhu et al. [107] have reported values of 1.9 and $2.1 \mathrm{eV}$ for the indirect and direct band gap, respectively, of $\mathrm{ZnFe}_{2} \mathrm{O}_{4}$ samples possessing oxygen vacancies in an unknown quantity. Higher indirect and direct band gap values of approximately 2.0 and $2.3 \mathrm{eV}$, respectively, were observed for pristine $\mathrm{ZnFe}_{2} \mathrm{O}_{4}$ in which oxygen vacancies were not detected [108]. These experimental results also support the theoretical results and, therefore, it becomes clear that oxygen vacancies have a considerable impact on the band gap energy of $\mathrm{ZnFe}_{2} \mathrm{O}_{4}$. It is well known that oxygen vacancies play a fundamental role in the photoelectrochemical efficiency of ferrites $[107,109]$. Therefore, understanding the impact of oxygen vacancies on the band gap energy is of utmost importance both from a theoretical and an experimental point of view. 
Tab. 5: Experimental band gaps of $\mathrm{MFe}_{2} \mathrm{O}_{4}(M=\mathrm{Mg}, \mathrm{Mn}-\mathrm{Zn})$, measurement temperature, method and morphology of probe.

\begin{tabular}{|c|c|c|c|c|c|}
\hline$M$ & $\mathrm{E}_{g}^{\exp }[\mathrm{eV}]$ & $\mathbf{T}$ & Method & Material & Reference \\
\hline \multirow[t]{6}{*}{$\overline{M g}$} & 1.85 & * & UV-Vis DRS & nanoparticles $(\sim 56.9 \mathrm{~nm})$ & [69] \\
\hline & 1.94 & * & UV-Vis DRS & nanoparticles $(\sim 41 \mathrm{~nm})$ & [70] \\
\hline & 2.00 & * & UV-Vis DRS & nanoparticles $(\sim 25 \mathrm{~nm})$ & [71] \\
\hline & 2.14 & * & UV-Vis DRS & nanoparticles $(\sim 17-22 \mathrm{~nm})$ & [72] \\
\hline & 2.175 & * & UV-Vis absorption & nanoparticles $(\sim 200 \pm 50 \mathrm{~nm})$ & [73] \\
\hline & 2.43 & * & UV-Vis absorption & nanoparticles $(\sim 4.9-9.3 \mathrm{~nm})$ & [74] \\
\hline \multirow[t]{6}{*}{$\mathrm{Mn}$} & 0.98 & * & UV-Vis absorption & nanoparticles $(\sim 47.3 \mathrm{~nm})$ & [76] \\
\hline & 1.68 & * & UV-Vis DRS & hollow nanospheres $(170-220 \mathrm{~nm})$ & [77] \\
\hline & 1.74 & * & UV-Vis DRS & colloidal nanocrystal clusters & [77] \\
\hline & 2.1 & RT & UV-Vis absorption & thin films $(\sim 150 \mathrm{~nm})$ & a \\
\hline & 2.15 & * & UV-Vis DRS & nanoparticles $(\sim 43.56 \mathrm{~nm})$ & [78] \\
\hline & 2.37 & * & UV-Vis DRS & nanoparticles $(\sim 15.42 \mathrm{~nm})$ & [78] \\
\hline \multirow[t]{2}{*}{$\mathrm{Fe}$} & 0.12 & * & UV-Vis absorption & nanoparticles & [79] \\
\hline & 1.92 & * & UV-Vis absorption & nanoparticles $(<20 \mathrm{~nm})$ & [80] \\
\hline \multirow[t]{13}{*}{ Co } & $1.17 \pm 0.08$ & * & UV-Vis DRS & thin films & [82] \\
\hline & 1.3 & * & DRS: HREELS & thin films & [83] \\
\hline & 1.36 & * & UV-Vis DRS & mesoporous powder & [84] \\
\hline & 1.37 & * & UV-Vis absorption & nanoparticles ( $29 \mathrm{~nm})$ & [85] \\
\hline & 1.41 & * & UV-Vis absorption & thin films $(\sim 23 \mathrm{~nm})$ & [86] \\
\hline & 1.42 & RT & ellipsometry & thin films $(\sim 80.4 \mathrm{~nm})$ & [87] \\
\hline & 1.58 & * & UV-Vis absorption & thin films $(\sim 16 \mathrm{~nm})$ & [86] \\
\hline & 2.2 & RT & UV-Vis absorption & thin films $(\sim 150 \mathrm{~nm})$ & a \\
\hline & 2.44 & * & UV-Vis absorption & nanoparticles $(\sim 14 \mathrm{~nm})$ & [81] \\
\hline & 2.505 & RT & UV-Vis absorption & thin films $(\sim 58.1 \mathrm{~nm})$ & [88] \\
\hline & 2.556 & RT & UV-Vis absorption & thin films $(\sim 36.3 \mathrm{~nm})$ & [88] \\
\hline & 2.59 & * & UV-Vis DRS & nanoparticles $(\sim 18 \mathrm{~nm})$ & [72] \\
\hline & 2.615 & RT & UV-Vis absorption & thin films $(\sim 5.8 \mathrm{~nm})$ & [88] \\
\hline \multirow[t]{13}{*}{$\mathrm{Ni}$} & $1.52 \pm 0.08$ & * & DRS: HREELS & thin films & [83] \\
\hline & 1.56 & RT & UV-Vis DRS & nanoparticles $(\sim 38 \mathrm{~nm})$ & [89] \\
\hline & 1.57 & * & UV-VIS DRS & nanoparticles $(\sim 20 \mathrm{~nm})$ & [90] \\
\hline & $1.64 \pm 0.08$ & $300 \mathrm{~K}$ & UV-Vis DRS & thin films $(150-270 \mathrm{~nm})$ & [15] \\
\hline & 1.65 & RT & ellipsometry & thin films $(\sim 69.3 \mathrm{~nm})$ & [87] \\
\hline & 1.77 & * & UV-Vis absorption & nanoparticles $(\sim 43.72 \mathrm{~nm})$ & [91] \\
\hline & 1.77 & * & UV-Vis absorption & nanoparticles $(\sim 35 \mathrm{~nm})$ & [85] \\
\hline & 2.0 & RT & UV-Vis absorption & thin films $(\sim 150 \mathrm{~nm})$ & 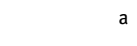 \\
\hline & 2.02 & * & UV-Vis DRS & nanoparticles ( 19 nm) & [92] \\
\hline & 2.19 & * & UV-Vis DRS & nanoparticles $(\sim 25.7 \mathrm{~nm})$ & [93] \\
\hline & 2.5 & RT & UV-Vis DRS & nanoparticles $(\sim 25 \mathrm{~nm})$ & [94] \\
\hline & 2.7 & * & UV-Vis absorption & mesoporous thin films $(\sim 16 \mathrm{~nm})$ & [95] \\
\hline & 3.54 & * & UV-Vis absorption & nanoparticles $(\sim 9 \mathrm{~nm})$ & [81] \\
\hline
\end{tabular}


Tab. 5 (continued)

\begin{tabular}{rrlllr}
\hline $\boldsymbol{M}$ & $\mathbf{E}_{g}^{\text {exp }}[\mathrm{eV}]$ & $\mathbf{T}$ & Method & Material & Reference \\
\hline $\mathrm{Cu}$ & 1.37 & $*$ & UV-Vis DRS & mesoporous powder & {$[84]$} \\
& 1.42 & $*$ & UV-Vis DRS & nanoparticles $(\sim 43 \mathrm{~nm})$ & {$[96]$} \\
& 1.54 & $*$ & UV-Vis DRS & nanoparticles $(\sim 74 \mathrm{~nm})$ & {$[97]$} \\
& 1.59 & $*$ & UV-Vis absorption & nanoparticles $(\sim 13.85 \mathrm{~nm})$ & {$[91]$} \\
& 1.61 & $*$ & UV-Vis absorption & nanoparticles $(\sim 41 \mathrm{~nm})$ & {$[85]$} \\
& 2.0 & RT & UV-Vis absorption & thin films $(\sim 150 \mathrm{~nm})$ & a \\
& 1.78 & $*$ & UV-Vis DRS & mesoporous powder & {$[84]$} \\
& 1.83 & RT & UV-Vis DRS & nanoparticles $(29-47 \mathrm{~nm})$ & {$[98]$} \\
& 1.923 & $*$ & UV-Vis DRS & nanoparticles & {$[99]$} \\
& 2.01 & $*$ & UV-Vis absorption & nanoparticles $(\sim 26 \mathrm{~nm})$ & {$[85]$} \\
& 2.61 & $*$ & UV-Vis absorption & sputtered thin films $(\sim 200 \mathrm{~nm})$ & {$[100]$} \\
& 2.88 & $296 \mathrm{~K}$ & ellipsometry & thin films $(90-150 \mathrm{~nm})$ & {$[101]$} \\
& 3.25 & $*$ & UV-Vis absorption & nanoparticles $(\sim 12 \mathrm{~nm})$ & {$[81]$} \\
\hline
\end{tabular}

$\mathrm{T}=$ * indicates that the measurement temperature was not provided, but is most probably room temperature (RT).

${ }^{\text {a }}$ L. I. Granone, R. Dillert, and D. W. Bahnemann, unpublished data.

\subsection{Influence of inversion on Raman spectra}

\subsubsection{Theoretical results}

Not only the dielectric constant and band gap but also vibrational spectra are influenced by the cation distribution. In this section we discuss the influence of inversion on the Raman spectrum of $\mathrm{ZnFe}_{2} \mathrm{O}_{4}$. This was investigated in a combined experimental and theoretical study [108]. The calculated spectra will be compared with measurements in Section 3.2.2.

The structure of antiferromagnetic $\mathrm{ZnFe}_{2} \mathrm{O}_{4}$ was optimized for degrees of inversion of $x=0$ and 1 . Since the present version of CRYSTAL does not allow for CPKS calculations with HSE06, only peak positions were calculated with this functional, while intensities were determined using the B3LYP functional, a temperature of $293.15 \mathrm{~K}$ and a laser wavelength of $785 \mathrm{~nm}$. For all calculations the rev2-POB-TZVP basis sets were employed. More details on the calculations can be found in reference [108]. Figure 3 shows the calculated Raman spectra of $\mathrm{ZnFe}_{2} \mathrm{O}_{4}$ for $x=0$ and 1 . The main peak for $x=0$ at $375 \mathrm{~cm}^{-1}$ shifts to smaller wavenumbers $\left(325 \mathrm{~cm}^{-1}\right)$ under inversion and the intensity is decreased. A double peak at $464 \mathrm{~cm}^{-1}$ and $472 \mathrm{~cm}^{-1}$ for $x=0$ slightly shifts to smaller wavenumbers and is the most intense signal for $x=1$. Another striking feature of the Raman spectrum for $x=1$ is the threefold signal at $541 \mathrm{~cm}^{-1}, 563 \mathrm{~cm}^{-1}$ and $586 \mathrm{~cm}^{-1}$. For a more detailed analysis of the spectra, please consult reference [108]. 


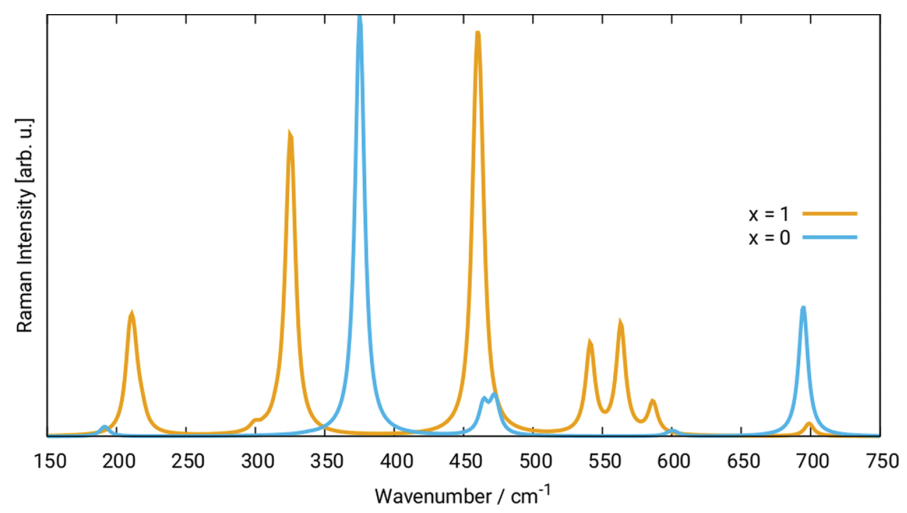

Fig. 3: Calculated Raman spectrum of antiferromagnetic $\mathrm{ZnFe}_{2} \mathrm{O}_{4}$ with $x=0$ and $x=1$.

One of the main problems in experimental literature for spinel ferrites is, that the degree of inversion is often not determined. Table 1 in Section 3.1.1 shows that the energetic difference between normal and inverse $\mathrm{ZnFe}_{2} \mathrm{O}_{4}$ is only $12 \mathrm{~kJ} / \mathrm{mol}$ according to our calculations. While this $\Delta E$ is enough to classify inverse $\mathrm{ZnFe}_{2} \mathrm{O}_{4}$ as metastable, it still is low enough to allow inversion during synthesis. As shown in the section above, the cation distribution crucially influences the electronic structure of the material, and thus will influence the photocatalytic performance. In the present section it was shown that Raman spectroscopy can be a tool to estimate the degree of inversion.

\subsubsection{Experimental results}

Raman spectroscopy is a convenient experimental technique for the characterization of ferrites due to its suitability towards the qualitative estimation of the degree of inversion. Several theoretical and experimental reports concerning the Raman study of the cation distribution on ferrites are found in the scientific literature [108, 110-113]. For normal $\mathrm{ZnFe}_{2} \mathrm{O}_{4}$, distinctive changes are expected to be observed in the Raman scattering as the degree of inversion increases, i.e. $\mathrm{Fe}^{3+}$ cations placed in octahedral sites $\left(\mathrm{FeO}_{6}\right)$ and $\mathrm{Zn}^{2+}$ cations placed in tetrahedral sites $\left(\mathrm{ZnO}_{4}\right)$ are interchanged [108]. These changes in the Raman scattering originate due to two effects. On the one hand, additional vibrational modes arise due to the formation of $\mathrm{ZnO}_{6}$ and $\mathrm{FeO}_{4}$ structures. On the other hand, a loss of symmetry, as a consequence of the cation inversion, results in the splitting of the Raman active internal modes of normal $\mathrm{ZnFe}_{2} \mathrm{O}_{4}[114,115]$. Figure $4 \mathrm{a}$ shows the normalized Raman spectra of $\mathrm{ZnFe}_{2} \mathrm{O}_{4}$ samples synthesized by a solid-state method exhibiting particle sizes of around $300 \mathrm{~nm}$ and with degrees of inversion 

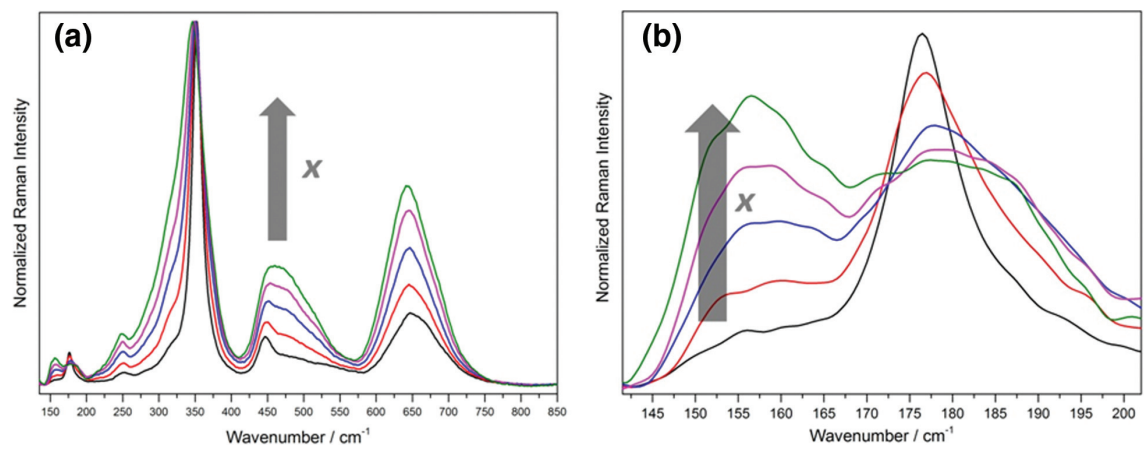

Fig. 4: Normalized Raman spectra of $\mathrm{ZnFe}_{2} \mathrm{O}_{4}$ samples with degrees of inversion increasing from $x \approx 0.07$ (black line) to $x \approx 0.20$ (green line). The spectra shown in red, blue and magenta correspond to $\mathrm{ZnFe}_{2} \mathrm{O}_{4}$ samples having $x \approx 0.10,0.13$, and 0.16 , respectively. (b) Magnification of the spectral region comprised between 141.5 and $202 \mathrm{~cm}^{-1}$ of the Raman spectra shown in (a). The spectra were measured using a $785 \mathrm{~nm}$ laser as excitation source. Adapted from reference [108] with permission from the PCCP Owner Societies.

increasing from $x \approx 0.07$ to $x \approx 0.20$ [108]. Changes in the relative intensities and a broadening of the Raman scattering signals become obvious by observing the spectra shown in Figure 4a. In order to analyze these effects and compare them with the theoretical predictions, the spectral region between 141.5 and $202 \mathrm{~cm}^{-1}$ (Figure 4b) will be discussed in more detail here. Nevertheless, similar interpretations are obtained by analyzing the entire Raman spectrum, as it is reported in reference [108]. It is observed from Figure $4 \mathrm{~b}$ that, as the degree of inversion increases, the intensity of the signal present at $156 \mathrm{~cm}^{-1}$ for $x \approx 0.07$ increases, whereas the intensity of the signal observed at $176.5 \mathrm{~cm}^{-1}$ decreases. This decrease is accompanied by a growth of a shoulder at higher wavenumbers, resulting in a broad band from $168 \mathrm{~cm}^{-1}$ to $198 \mathrm{~cm}^{-1}$. The occurrence of the signal at $156 \mathrm{~cm}^{-1}$ was attributed to the symmetry distortion as a consequence of the increasing degree of inversion. In addition, the signal located at $176.5 \mathrm{~cm}^{-1}$ for $x \approx 0.07$ was attributed to the active mode predicted at $191 \mathrm{~cm}^{-1}$ by the theoretical calculations, see Figure 3. It is worth mentioning that vibrational wavenumbers obtained from DFT calculations are typically larger than the ones obtained experimentally [116, 117]. Therefore, deviations below $20 \mathrm{~cm}^{-1}$ are considered accurate $[108,116]$. As was mentioned above, the theoretical calculations predict a shift of the vibrational mode at $191 \mathrm{~cm}^{-1}$ towards higher wavenumbers upon inversion. This theoretical prediction is in excellent agreement with the experimental data because an analogous behavior is observed for the signal at $176.5 \mathrm{~cm}^{-1}$. For further insight concerning the correlation between calculated 
and experimentally obtained Raman spectra of $\mathrm{ZnFe}_{2} \mathrm{O}_{4}$, and their dependence on the degree of inversion, the reader is referred to reference [108].

In the literature it is reported that the degree of inversion of $\mathrm{ZnFe}_{2} \mathrm{O}_{4}$ might be estimated by analyzing the Raman signal located around $650 \mathrm{~cm}^{-1}$ [118-120]. Some authors claim that this band, attributed to the $\mathrm{A}_{1 g}$ Raman active mode, originates from vibrations involving oxygen atoms in $\mathrm{MO}_{4}$ environments. Thus, for partially inverted $\mathrm{ZnFe}_{2} \mathrm{O}_{4}$, the $\mathrm{A}_{1 g}$ band is deconvoluted in two different signals ascribed to vibrations involving oxygen atoms in $\mathrm{ZnO}_{4}$ and $\mathrm{FeO}_{4}$ structures, respectively. Finally, a one-to-one relationship between the degree of inversion and the intensity ratio of these two components is made. The assignment of the signal located around $650 \mathrm{~cm}^{-1}$ to vibrations involving oxygen atoms in the $\mathrm{MO}_{4}$ structures was made by White and DeAngelis [114]. They reached this conclusion by the artifice of pretending that the spinel structure $\mathrm{A}_{2} \mathrm{BO}_{4}$ is composed of four A ions and two $\mathrm{BO}_{4}$ molecules [114]. As White and DeAngelis stated in their manuscript, this is an accurate approximation only in extreme cases, such as $\mathrm{Na}_{2} \mathrm{WO}_{4}$, in which the $\mathrm{W}-\mathrm{O}$ bonding is largely covalent. Furthermore, the Raman signals due to vibrations involving $\mathrm{W}-\mathrm{O}$ bonds are much stronger than those involving $\mathrm{Na}-\mathrm{O}$ bonds. A similar assumption is not valid for $\mathrm{ZnFe}_{2} \mathrm{O}_{4}$. Our theoretical calculations showed that the Raman band with $\mathrm{A}_{1 g}$ symmetry expected at around $695 \mathrm{~cm}^{-1}$ is attributed to vibrations involving oxygen atoms in both $\mathrm{MO}_{4}$ and $\mathrm{MO}_{6}$ structures [108]. Furthermore, even though the experimental $\mathrm{A}_{1 g}$ band located around $650 \mathrm{~cm}^{-1}$ in the Raman spectra shown in Figure 4 can be deconvoluted in at least two different signals [108], there is no reasonable evidence for a one-to-one relationship between the degree of inversion and the intensity ratio of the deconvoluted components. The first argument against this relationship is that the $\mathrm{A}_{1 g}$ mode shows contributions from both $\mathrm{MO}_{4}$ and $\mathrm{MO}_{6}$ structures. The second and most important argument is that the relative abundance of the $\mathrm{ZnO}_{4}$ and $\mathrm{FeO}_{4}$ units is not necessarily equal to the relative intensity of their Raman signals. This is due to the different Raman scattering properties of the $\mathrm{ZnO}_{4}$ and $\mathrm{FeO}_{4}$ structures. Clear evidence of this fact is observed in Figure 3, where a large decrease in the intensity of the $\mathrm{A}_{1 g}$ signal is predicted by the theoretical calculations as the degree of inversion shifts from 0 to 1 . Furthermore, the Raman scattering intensity is wavelength-dependent and, therefore, the intensity ratio will be different depending on the excitation wavelength used for the measurement [108]. The quantitative rather than qualitatively estimation of the degree of inversion from Raman spectroscopy would be highly desirable. However, additional experimental techniques such as Mößbauer spectroscopy or XRD plus structural refinement are necessary.

It is worth noting that Raman spectroscopy is a powerful experimental technique for the characterization of $\mathrm{ZnFe}_{2} \mathrm{O}_{4}$ samples because it allows the detection 
of maghemite $\left(\gamma-\mathrm{Fe}_{2} \mathrm{O}_{3}\right)$ present as a secondary phase. As well as $\mathrm{ZnFe} \mathrm{O}_{4}, \gamma-\mathrm{Fe}_{2} \mathrm{O}_{3}$ exhibits a spinel crystal structure and, therefore, these materials are not easily distinguished by means of XRD. Furthermore, it is well known that nanoparticulate $\mathrm{ZnFe}_{2} \mathrm{O}_{4}$ forms solid solutions with $\gamma-\mathrm{Fe}_{2} \mathrm{O}_{3}$ in all proportions without losing the single-phase spinel structure [121]. The physicochemical properties and the photoelectrochemical activity of the $\mathrm{ZnFe}_{2} \mathrm{O}_{4} / \gamma-\mathrm{Fe}_{2} \mathrm{O}_{3}$ solid solutions significantly differ from those of the pristine $\mathrm{ZnFe}_{2} \mathrm{O}_{4}$ [122]. Therefore, it is of utmost importance to detect whether $\gamma-\mathrm{Fe}_{2} \mathrm{O}_{3}$ impurities are present or not in order to properly characterize $\mathrm{ZnFe}_{2} \mathrm{O}_{4}$ samples. Other analytical techniques, such as Mößbauer spectroscopy, also allow the detection of $\gamma-\mathrm{Fe}_{2} \mathrm{O}_{3}$ impurities. However, these analytical methods are not as accessible as Raman spectroscopy, which is currently considered a routine spectroscopy technique and is normally available in most research laboratories. Figure 5 shows the Raman spectrum of $\mathrm{ZnFe}_{2} \mathrm{O}_{4}$ with a degree of inversion $x \approx 0.07$ and of $\gamma-\mathrm{Fe}_{2} \mathrm{O}_{3}$. Even though most of the Raman scattering signals are superimposed, $\gamma-\mathrm{Fe}_{2} \mathrm{O}_{3}$ is readily differentiated from $\mathrm{ZnFe}_{2} \mathrm{O}_{4}$ by the broad signal observed at approximately $718 \mathrm{~cm}^{-1}[108,123]$.

The experimental results presented in this section highlight the versatility and suitability of Raman spectroscopy towards the characterization of $\mathrm{ZnFe}_{2} \mathrm{O}_{4}$. Valuable information concerning both the purity and the degree of inversion

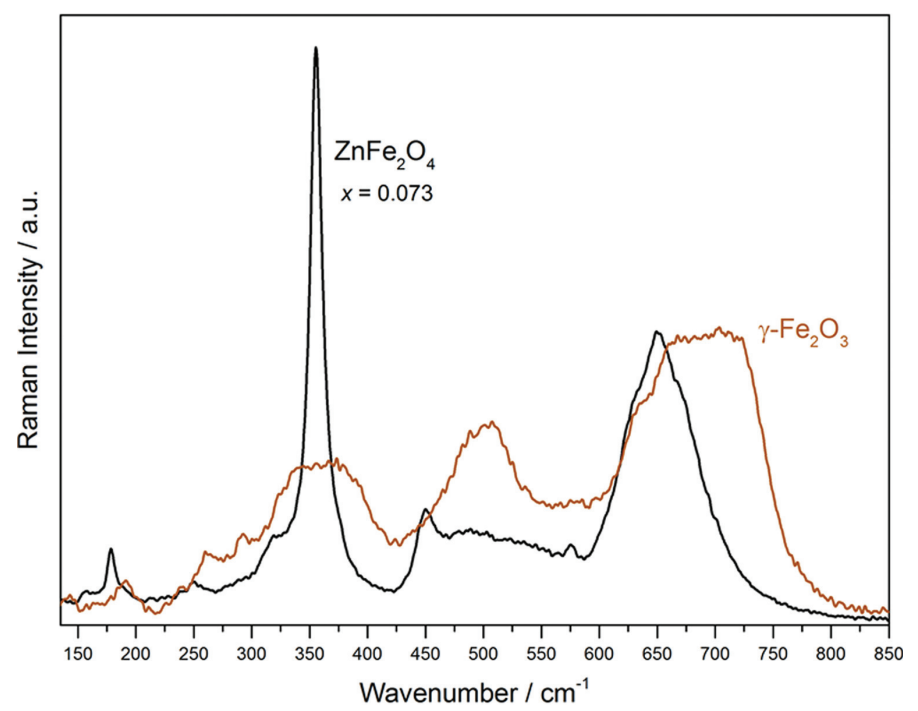

Fig. 5: Raman spectrum of $\mathrm{ZnFe}_{2} \mathrm{O}_{4}\left(x \approx 0.07\right.$, black line) and $\gamma-\mathrm{Fe}_{2} \mathrm{O}_{3}$ (brown line) measured using a $633 \mathrm{~nm}$ laser as excitation source. Reproduced from reference [108] with permission from the PCCP Owner Societies. 
of $\mathrm{ZnFe}_{2} \mathrm{O}_{4}$ samples can be extracted from a careful Raman analysis. Furthermore, the combination of data obtained from theoretical and experimental studies allows gaining fundamental knowledge concerning the nature of the atomic vibrations responsible for the observed Raman modes and their dependence on the degree of inversion.

Whereas experimental data of Raman measurements of zinc ferrite with lower degree of inversion are available (inversion $x<0.2$ ) [93], the availability of data for higher degrees of inversion are scarce due to experimental limitations [108]. The degree of inversion increases with the annealing temperature and for samples with an inversion of $x>0.5$ temperatures of $2000 \mathrm{~K}$ or more are required $[108,124]$.

Recently, we have demonstrated that the electrodeposition of zinc ferrite films is a versatile platform to vary the $\mathrm{Zn}$ :Fe ratio leading to zinc ferrite films with varied degree of inversion and have performed Raman measurements with those samples [122]. Zinc ferrite samples with a high degree of inversion exhibit a high amount of iron on the tetrahedral sites of the crystal structure. The electrodeposited zinc ferrite films deviate from the ideal stoichiometric composition of $\mathrm{ZnFe}_{2} \mathrm{O}_{4}$ and are either zinc enriched ( $\mathrm{Zn}: \mathrm{Fe}$ ratio $>0.5$ ) or zinc depleted ( $\mathrm{Zn}: \mathrm{Fe}$ ratio $<0.5)$. However, attempts to quantify the degree of inversion for these samples using XRD are not reliable due to overlapping reflexes of the required conductive support (FTO). Thus comparison of experimental Raman spectra with theoretically simulated spectra may give a useful hint.

In literature the reported Raman spectra slightly deviate from the theoretically calculated (Figure 3) and often the degree of inversion is not reported. Wang et al. [125] reported five Raman signals for zinc ferrite with signals at $647 \mathrm{~cm}^{-1}$, $451 \mathrm{~cm}^{-1}, 355 \mathrm{~cm}^{-1} 246 \mathrm{~cm}^{-1}$ and $221 \mathrm{~cm}^{-1}$, respectively, for a laser wavelength of $785 \mathrm{~nm}$. The signal at $647 \mathrm{~cm}^{-1}$ is attributed to the vibrational motion between oxygen and metal on the tetrahedral site with $A_{1_{g}}$ symmetry. All other signals were attributed to octahedral sites with $E_{g}\left(246 \mathrm{~cm}^{-1}\right)$ or $F_{2 g}\left(451 \mathrm{~cm}^{-1}, 355 \mathrm{~cm}^{-1}\right.$, $221 \mathrm{~cm}^{-1}$ ) symmetry. Here one can note a difference in interpretation: the signal at $451 \mathrm{~cm}^{-1}$ is attributed by Wang et al. to an octahedral site, however, in the theoretical calculations this is assigned to wagging modes of tetrahedrally coordinated atoms. Furthermore, the signals between $200 \mathrm{~cm}^{-1}$ and $400 \mathrm{~cm}^{-1}$ were also attributed to octahedral sites but theoretical findings indicate that both tetrahedral and octahedral sites have an influence on those signals.

At the data given by Wang et al. [125] the signal at $355 \mathrm{~cm}^{-1}$ is by far the signal with the highest intensity whereas the signals at $221 \mathrm{~cm}^{-1}$ and $246 \mathrm{~cm}^{-1}$ are hardly detectable. All signals show a slight signal broadening which is explained due a low degree of inversion without giving precise numbers. Nevertheless the 
Raman spectrum reported by Wang et al. is very similar to the calculated spectrum for an inversion of $x=0$.

For electrochemically prepared zinc ferrite films (as seen in Figure 6), the signal for the tetrahedral wagging at $\sim 200 \mathrm{~cm}^{-1}$ is weak for all $\mathrm{Zn}$ :Fe ratios. The signal is positioned at $190 \mathrm{~cm}^{-1}$ and the signal becomes less distinct with increasing zinc content. For the zinc enriched film no signal is detectable. This suggests that zinc depleted samples behave like zinc ferrite samples with a high degree of inversion as predicted theoretically. In the theoretical Raman spectrum the tetrahedral wagging signal has distinct intensity for $x=1$, but negligible for $x=0$.

An additional effect of $\mathrm{Zn}$ :Fe ratio variation is the striking difference of the width of the signal at $355 \mathrm{~cm}^{-1}$. The highly zinc depleted sample shows

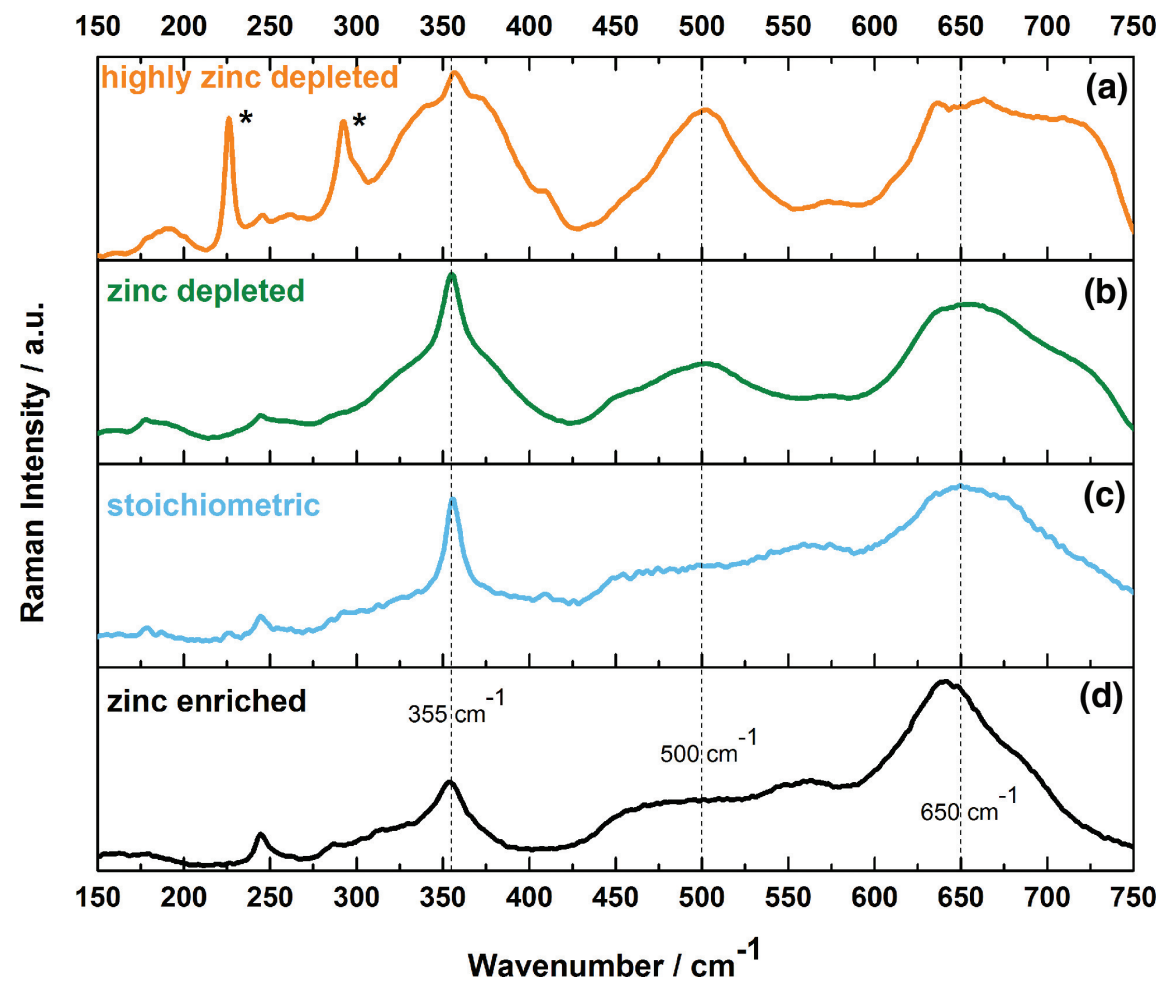

Fig. 6: Raman spectra of zinc ferrite film samples with different stoichiometry. (a) Zn:Fe ratio $=0.1$, (b) $\mathrm{Zn}$ :Fe ratio $=0.25$, (c) $\mathrm{Zn}: \mathrm{Fe}$ ratio $=0.5$ as it appears in zinc ferrite with ideal $\mathrm{ZnFe}_{2} \mathrm{O}_{4}$ stoichiometry, and (d) $\mathrm{Zn}$ :Fe ratio $=1.0$. Asterisks indicate signals of Hematite $\left(\alpha-\mathrm{Fe}_{2} \mathrm{O}_{3}\right)$ traces. All given ratios are surface ratios of the film samples as measured by XPS. The dotted lines are guides to the eye with given wavenumber position. 
specifically broad signal with shoulders. Compared with the theoretical spectrum this can be interpreted as an overlap of both signals of the fully inverted $(x=1)$ and non-inverted $(x=0)$ sample. With increasing zinc amount the width of the signal gradually decreases. The position of this signal is not remarkably shifted for all stoichiometric variations.

Another distinctive difference is the signal intensity at $500 \mathrm{~cm}^{-1}$. For the highly zinc depleted sample instead of a sharp signal at $460 \mathrm{~cm}^{-1}$ and a triple signal at $\sim 550 \mathrm{~cm}^{-1}$, as expected from the theoretical calculation, one broad signal was observed. The signal intensity decreases with higher zinc content and it is no longer detectable for the stoichiometric sample and the zinc enriched sample. This observation is in accordance with the theory and suggests that a zinc depleted zinc ferrite film behaves similar to a sample with a high degree of inversion. Samples with $x=0$ have almost no signal intensity in the wavenumber range $430 \mathrm{~cm}^{-1}-650 \mathrm{~cm}^{-1}$ and therefore for the stoichiometric and zinc enriched electrodeposited films no distinct signal is detected in this wavenumber range.

Furthermore, the measured signals between $600 \mathrm{~cm}^{-1}$ and $700 \mathrm{~cm}^{-1}$ show different features compared to the theoretical Raman spectrum. Following the argument that a zinc depleted sample behaves similar to zinc ferrite with a high degree of inversion $(x=1)$, no signal or just a weak signal should be visible in this wavenumber range. For a stoichiometric sample the signal at this wavenumber position should become sharper. Whereas for the stoichiometric sample (Figure 6, blue line) and the zinc enriched sample (Figure 6, black line) the FWHM decreases and the signal becomes more defined and the behavior is consistent with the theoretical calculations, no explanation can be given from theoretical point of view regarding the particularly broad signal for the highly zinc depleted sample (Figure 6, orange line).

This behavior of the Raman signal at $\sim 650 \mathrm{~cm}^{-1}$ for zinc ferrite samples with different stoichiometry was also mentioned by other authors [126, 127]. Rivero et al. [126] observed a distinctive signal at $667 \mathrm{~cm}^{-1}$ for a zinc ferrite sample with $\mathrm{Zn}_{0.93} \mathrm{Fe}_{2.07} \mathrm{O}_{4}$ stoichiometry. For a zinc depleted sample with $\mathrm{Zn}_{0.30} \mathrm{Fe}_{2.70} \mathrm{O}_{4}$ stoichiometry the intensity of this signal deceases and forms a broad doublet. This behavior is explained by the fact that not just $\mathrm{Zn}^{2+}$ (for $x=0$ ) or $\mathrm{Fe}^{3+}$ (for $x=1$ ) are located at the tetrahedral sites of zinc ferrite but a mixture of both ions leading to signal overlap and therefore broadening of the signal. The $\mathrm{Zn}-\mathrm{O}$ stretching seems to be more Raman active so that the signal intensity decreases with increasing iron amount. For samples with low $\mathrm{Zn}$ content the detected hematite impurities do also have an influence on the signal broadening in this wavenumber range. In our previous work [122] we also detected $\mathrm{Fe}^{2+}$ ions in the zinc depleted and highly zinc depleted samples by XPS. Therefore also $\mathrm{Fe}^{2+}-\mathrm{O}$ vibrations could have an influence on the signal broadening. 
In summary for the electrodeposited zinc depleted, stoichiometric, and zinc enriched ferrite films the Raman spectra are quite similar to theoretically calculated spectra of zinc ferrite with inversion and without inversion, respectively. The slight deviations may be explained by phase impurities of hematite and signal broadening due to temperature, laser wavelength and laser intensity used in the Raman measurements [128].

Another remarkable fact is that for our electrodeposited films the Raman spectra of the stoichiometric and zinc enriched sample are very similar although the stoichiometry showed an obvious difference. Raman is a bulk technique and the penetration depth of the laser at least reaches $100 \mathrm{~nm}$ [129]. In our recent work [122] we showed by XPS analysis that the bulk of both samples is almost identical and zinc is only enriched at the top 20-30 $\mathrm{nm}$ of the zinc enriched sample.

\subsection{Optical spectra}

\subsubsection{Theoretical results}

For an assessment of the photoelectrochemical activity of $\mathrm{ZnFe}_{2} \mathrm{O}_{4}$, it is essential to obtain experimental information concerning its optical properties. The photon absorption is the first process in the complex chain of events occurring during a photoelectrochemical reaction [130], and the absorptivity of the material defines the maximum efficiency that can be expected [131]. The low-lying excited states that define the optical gap are usually composed of transitions from the highest occupied orbitals (HOCOs) into the lowest unoccupied orbitals (LUCOs) [132]. In addition to the band gap transition, electronic $d-d$ transitions are observed for $\mathrm{ZnFe}_{2} \mathrm{O}_{4}$ in the IR, visible, and UV range of the electromagnetic spectrum $[108,133]$.

To further study the influence of magnetic structure and the degree of inversion on $\mathrm{ZnFe}_{2} \mathrm{O}_{4}$ we calculated the dielectric function using GW/BSE. For this purpose, we used the plane waves program VASP [134]. Starting with a PBE ground-state calculation, a converged partially-selfconsistent sc $G W_{0}$-BSE algorithm was employed. The plane waves cutoff energy, number of bands, Monkhorst-Pack grid, GW cutoff energy and the number of frequency points (NOMEGA) have been converged carefully to $450 \mathrm{eV}, 960,4 \times 4 \times 4,200 \mathrm{eV}$ and 128 , respectively.

The relaxed structures of the most stable configurations of $\mathrm{ZnFe}_{2} \mathrm{O}_{4}, \mathrm{~A}-\mathrm{fm}$, A-afm1, and B-fi4, were used, as in Section 3.1.1. The configuration B-fi4 can be seen as an inverse version of A-afm1, since iron and zinc interchange positions, but the magnetic structure remains the same (cf. Table 2). B-fi4 is called ferrimagnetic, since iron atoms in tetrahedral positions couple with iron atoms in 
octahedral positions. Because these sites are not symmetry equivalent, the magnetic structure is technically ferrimagnetic, but still has a net magnetic moment of zero.

Figure 7 shows the imaginary part of the dielectric function in dependency of the excitation energy for the three configurations of $\mathrm{ZnFe}_{2} \mathrm{O}_{4}$. As can be seen, the maximum of the first excitation of antiferromagnetic normal $\mathrm{ZnFe}_{2} \mathrm{O}_{4}$ (A-afm1) is close to $3 \mathrm{eV}$. This is in good agreement with the DDSCH results obtained in Section 3.1.1, and some of the experimental literature discussed in Section 3.1.2. However, the excitation is rather broad and the onset is at $\sim 2 \mathrm{eV}$, which could be an explanation for experimental optical band gaps found in this region.

For inverse $\mathrm{ZnFe}_{2} \mathrm{O}_{4}$ (B-fi4), the onset of the first excitation does not shift significantly compared to $\mathrm{ZnFe}_{2} \mathrm{O}_{4}$ with normal cation distribution, but the intensity is reduced. A reduced intensity shows, that the material absorbs less light in this energy region, and thus will have an inferior photocatalytic performance.

For ferromagnetic normal $\mathrm{ZnFe}_{2} \mathrm{O}_{4}(\mathrm{~A}-\mathrm{fm})$, not only the intensity is reduced drastically compared to the antiferromagnetic structure, but also the onset of the first excitation shifts to higher energies. Compared to configuration A-afm1, the configuration A-fm will have a reduced photocatalytic performance, or will even be inactive.

By evaluating the optical spectra, we conclude that with increasing degree of inversion or by changing the magnetic structure, the photocatalytic performance of $\mathrm{ZnFe}_{2} \mathrm{O}_{4}$ is reduced drastically. Given that the configurations A-afm1 and A-fm are energetically very close (cf. Section 3.1.1) and the degree of inversion can easily

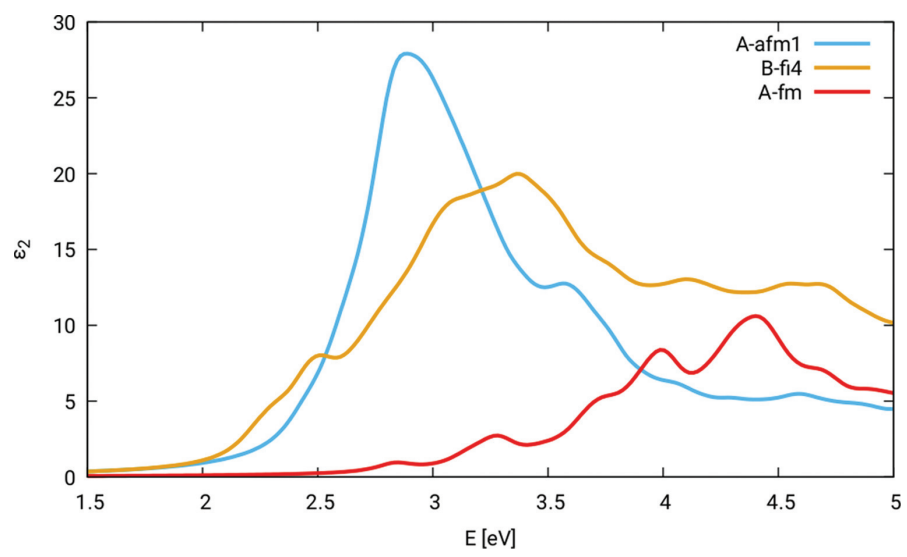

Fig. 7: Optical spectra of the most stable cation distribution and spin configurations of $\mathrm{ZnFe}_{2} \mathrm{O}_{4}$. 
be increased by the synthesis conditions, $\mathrm{ZnFe}_{2} \mathrm{O}_{4}$ is a problematic candidate as catalyst material.

In the comparison with experiment (see Section 3.3.2) it has to be kept in mind that the GW/BSE method does not describe $d$ - $d$ transitions (see Section 2.1.4). Low-lying bands in the experimental spectra could also be attributed to $\mathrm{Fe}$ $d-d$ transitions, although their intensities should be small due to the octahedral environment of Fe. Furthermore, intra-atomic transitions do not contribute to electron-hole separation and thus to photocatalytic activity.

\subsubsection{Experimental results}

The diffuse reflectance spectra of bulk $\mathrm{ZnFe}_{2} \mathrm{O}_{4}$ samples with degrees of inversion increasing from $x \approx 0.07$ to $x \approx 0.20$ are shown in Figure 8. It is worth noting that none of these samples exhibited signal attributed to $\mathrm{Fe}^{2+}$ cations in their Mößbauer spectra, which is an indication of the absence of oxygen

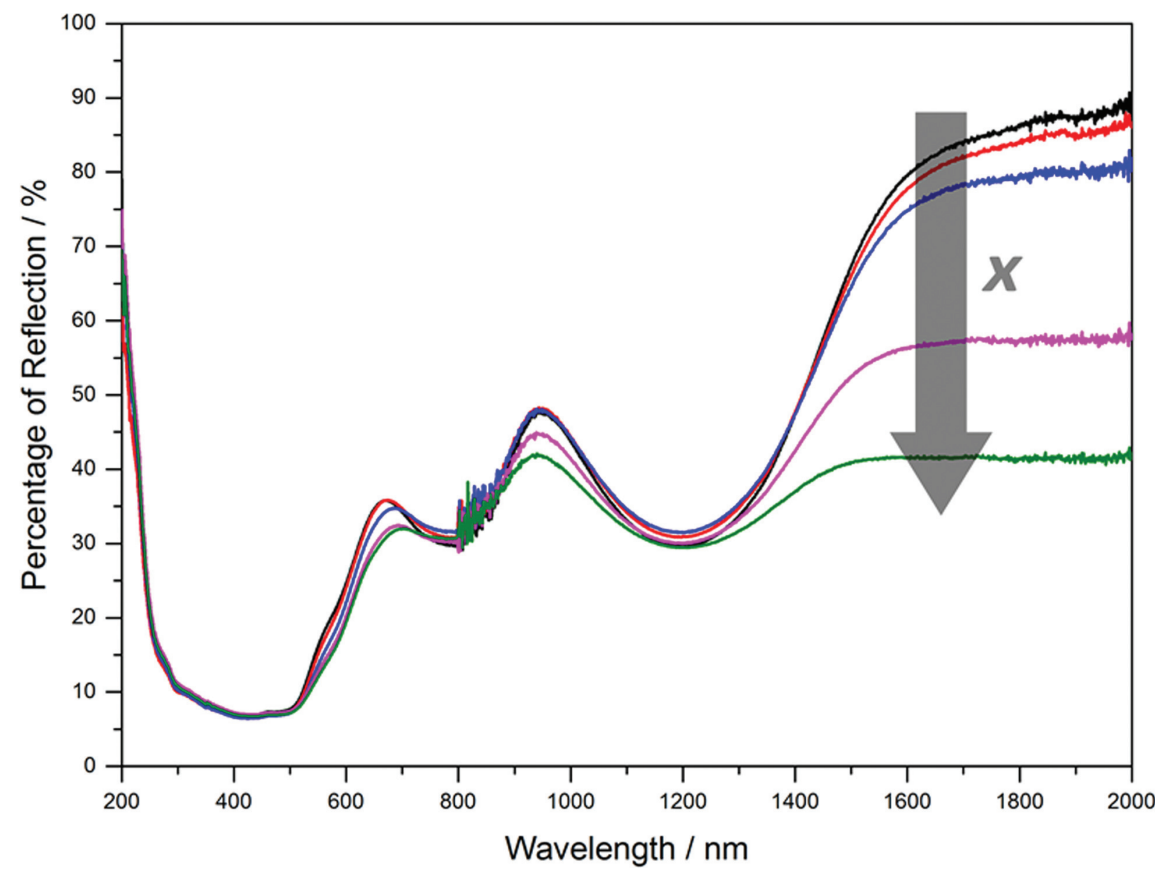

Fig. 8: UV-Vis-NIR diffuse reflectance spectra of $\mathrm{ZnFe}_{2} \mathrm{O}_{4}$ samples with degrees of inversion increasing from $x \approx 0.07$ (black line) to $x \approx 0.20$ (green line). The spectra shown in red, blue and magenta correspond to $\mathrm{ZnFe}_{2} \mathrm{O}_{4}$ samples having $x \approx 0.10,0.13$, and 0.16 , respectively. Reproduced from reference [108] with permission from the PCCP Owner Societies. 
vacancies [108]. Furthermore, Kelvin probe measurements showed negligible dopant concentrations, thus supporting the Mößbauer results [135]. The local minima observed in the spectra correspond to absorption maxima and, therefore, three predominant absorption bands are observed in the spectral regions from 220 to $670 \mathrm{~nm}, 670$ to $950 \mathrm{~nm}$, and 950 to $1600 \mathrm{~nm}$. The absorption maxima observed from 670 to $950 \mathrm{~nm}$ and 950 to $1600 \mathrm{~nm}$ are attributed to $d-d$ electronic transitions in $\mathrm{Fe}^{3+}$ cations, while the absorption maximum observed from 220 to $670 \mathrm{~nm}$ has the contribution of both, band gap and $d-d$ electronic transitions [108]. Within this region, the indirect and direct band gap are located at approximately $614 \mathrm{~nm}$ $(\approx 2.0 \mathrm{eV})$ and $532 \mathrm{~nm}(\approx 2.3 \mathrm{eV})$, respectively [107-109].

It is well known that $\mathrm{ZnFe}_{2} \mathrm{O}_{4}$ samples turn a darker color as the degree of inversion increases, even though the particle size is not modified [108, 124]. Determining whether this phenomenon is due to a band gap narrowing or to the contribution of $d-d$ transitions is fundamental to understand the impact of the degree of inversion on the optical properties of $\mathrm{ZnFe}_{2} \mathrm{O}_{4}$. Figure 9a shows the diffuse reflectance of the bulk $\mathrm{ZnFe}_{2} \mathrm{O}_{4}$ samples with increasing degree of inversion as a function of the photon energy. Deconvolution of the diffuse reflectance signals shows that, as the degree of inversion increases, the contribution of the $d-d$ electronic transition observed at $1.6 \mathrm{eV}(\approx 795 \mathrm{~nm})$ decreases whereas the signal corresponding to the transition observed at $1.0 \mathrm{eV}(\approx 1200 \mathrm{~nm})$ becomes wider [108]. Furthermore, by applying the derivation of absorption spectrum fitting (DASF) [136] method for the determination of the band gap, it was demonstrated that the band gap energies remain constant at $\approx 2.0 \mathrm{eV}$ and $\approx 2.3 \mathrm{eV}$, independently of the
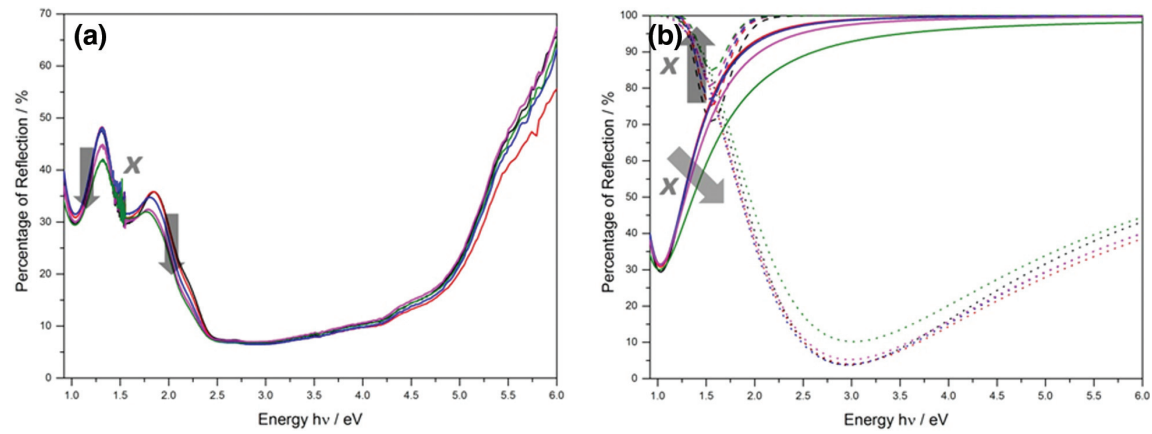

Fig. 9: (a) UV-Vis-NIR diffuse reflectance spectra from 0.9 to $6.0 \mathrm{eV}$ of $\mathrm{ZnFe}_{2} \mathrm{O}_{4}$ samples with degrees of inversion increasing from $x \approx 0.07$ (black line) to $x \approx 0.20$ (green line). The spectra shown in red, blue and magenta correspond to $\mathrm{ZnFe}_{2} \mathrm{O}_{4}$ samples having $x \approx 0.10,0.13$, and 0.16 , respectively. (b) Deconvolution of the UV-Vis-NIR diffuse reflectance spectra using Gaussian-shaped curves. Reproduced from reference [108] with permission from the PCCP Owner Societies. 
degree of inversion [108]. Therefore, the lower reflectivity, i.e. higher absorptivity, observed for $\mathrm{ZnFe}_{2} \mathrm{O}_{4}$ as the degree of inversion increases was attributed to contributions from $d-d$ electronic transitions rather than a band gap narrowing. This experimental observation is in agreement with the theoretical prediction showing that the band gap excitation energy of inverse $\mathrm{ZnFe}_{2} \mathrm{O}_{4}$ does not significantly shift compared to normal $\mathrm{ZnFe}_{2} \mathrm{O}_{4}$, and that the intensities of the interband transition decreases with increasing $x$.

In addition to the UV-Vis reflectance spectra of zinc ferrite powder with different degree of inversion, where we showed the influence of the inversion on the signal intensity, we also measured the optical spectra of zinc ferrite films with varying stoichiometry and compared the spectra with the calculated spectra (see Figure 7). The used samples are the same as discussed in Section 3.2.2. Due to slight variations in film thickness and film inhomogenities we avoid comparison of the signal intensity and focus on qualitative interpretations with normalized intensity.

The optical spectrum of an electrodeposited stoichiometric film (blue spectrum, Figure 10) has very similar features and the best agreement with the calculated optical spectra of A-afm1 (Figure 7) with antiferromagnetic spin configuration with ideal $\mathrm{Zn}$ :Fe ratio of 0.5 without inversion. The main optical transition for both, the theoretical model A-afm1 and the stoichiometric film, is located at around $2.8 \mathrm{eV}$ (note that main absorption transitions are located at the minima of

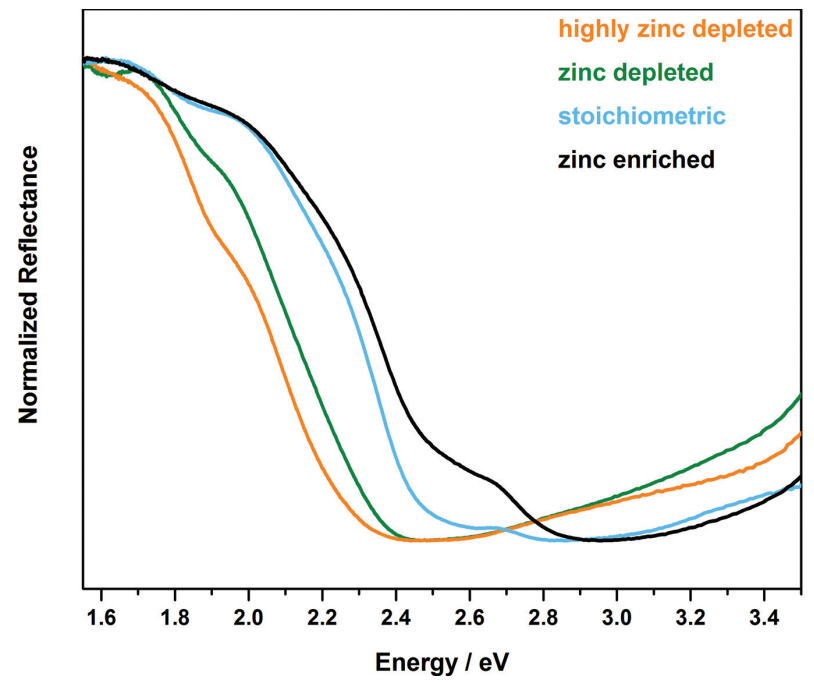

Fig. 10: Normalized UV-Vis diffuse reflectance spectra of electrodeposited zinc ferrite films with different stoichiometry. The colors codes and given stoichiometry are described in Figure 6. 
Figure 10). The main difference is that the signal for the experimental films is relatively broad with a shoulder at $2.6 \mathrm{eV}$. The onset of the optical transition is also quite similar at around $2.2 \mathrm{eV}$. The experimental optical spectrum of the stoichiometric film (blue spectrum, Figure 10) and the zinc enriched film (black spectrum, Figure 10) are very similar with the same onset of absorption.

For the zinc depleted samples the onset and the position of the main signal are shifted to lower energies. The zinc depleted sample has its minimum and therefore the main signal at $2.4 \mathrm{eV}$ with an onset at $\sim 1.9 \mathrm{eV}$. The highly zinc depleted sample is even further shifted to lower energies with its main signal at $2.3 \mathrm{eV}$ and onset at $\sim 1.7 \mathrm{eV}$. This trend is also observed for the calculated optical spectra for $\mathrm{B}$-fi4 (Figure 7) which is representative of an inverted $\mathrm{ZnFe}_{2} \mathrm{O}_{4}$ system.

From comparison of the calculated data with measured spectra we conclude that the inversion or stoichiometry has just a minor influence on the optical spectrum. A-afm1 and B-fi4 show very similar calculated spectra although they correspond to $x=0$ and $x=1$, respectively. The spectra of the stoichiometric sample and zinc depleted sample as representatives of samples without and with inversion are also similar to each other. Only the calculated spectrum for configuration A-fm (Figure 7) shows a significant difference compared with A-afm1 and B-fi4 or the experimental data. The main difference of the spectrum for A-fm compared with the other samples is its different spin state whereby the system is no longer antiferromagnetic but ferromagnetic. Those different spin states seem to have a stronger influence on the optical spectra than the degree of inversion. Unfortunately from our films we have no sample that resembles A-fm to support the beforehand stated conclusion as none of our prepared samples showed ferromagnetic behavior.

\subsection{Absolute band energies}

\subsubsection{Theoretical results}

In order to split water photocatalytically, the band gap has to be large enough (ca. $2.0 \mathrm{eV}$ ) to overcome the thermodynamic energy requirement $(1.23 \mathrm{eV})$ and the associated overpotentials to drive both the water oxidation and reduction reactions [137-139]. As shown in Section 3.1.1 this can be predicted via bulk calculations. Yet not only a suitable band gap is required, the absolute energies of the highest occupied and lowest unoccupied band of the semiconductor are equally essential for an efficient photocatalysis [140]. In this case the absolute band position refers to the vacuum reference and not to the system-dependent Fermi energy as common for the bulk. Due to an undefined reference of the oneparticle levels in 3D periodic calculations, the calculated absolute band energies 
of bulk structures have no physical meaning [141]. For absolute band energies, surface models are needed. To the best of the author's knowledge, only few investigations on low-index surfaces of $\mathrm{ZnFe}_{2} \mathrm{O}_{4}$ have been published [142-144]. Our investigations focus on the (100) surface of $\mathrm{ZnFe}_{2} \mathrm{O}_{4}$ since the (100) surface of normal spinels has been reported as most stable [61, 145]. In normal spinel oxides with divalent and trivalent cations, $\mathrm{AB}_{2} \mathrm{O}_{4}$, divalent cations are located in tetrahedral A-positions. Trivalent cations are located in octahedral B-positions. The (100) surface of $\mathrm{ZnFe}_{2} \mathrm{O}_{4}$ consists of alternating tetrahedral $\mathrm{Zn}$ layers and octahedral Fe layers. It can be terminated by a truncated tetrahedron, $\mathrm{ZnO}_{2}$, or two truncated octahedra, $\mathrm{FeO}_{5}$. A stoichiometric surface model as shown in Figure 11 is exposing both of these terminations, here the $\mathrm{ZnO}_{2}$ termination is shown in the topmost layer and the $\mathrm{FeO}_{5}$ termination is seen in the bottom layer. As discussed in Section 2.3, $\mathrm{ZnFe}_{2} \mathrm{O}_{4}(100)$ is a Tasker type 3 surface. The inherent dipole moment of surface unit cells causes problems in the SCF procedure and creates artifacts in the calculated electronic structure. We therefore followed various strategies to avoid the dipole moment in our models.

\subsubsection{Defective slab models}

Using the DDSCH method described in Chapter 3.1.1, we investigated defective, dipole free surface models. Starting from the optimized bulk structure denoted as A-afm1, see Table 2, defective surface models were created from a supercell of a symmetric $\mathrm{Zn}$-terminated slab. Half of the terminating $\mathrm{Zn}$ atoms were removed

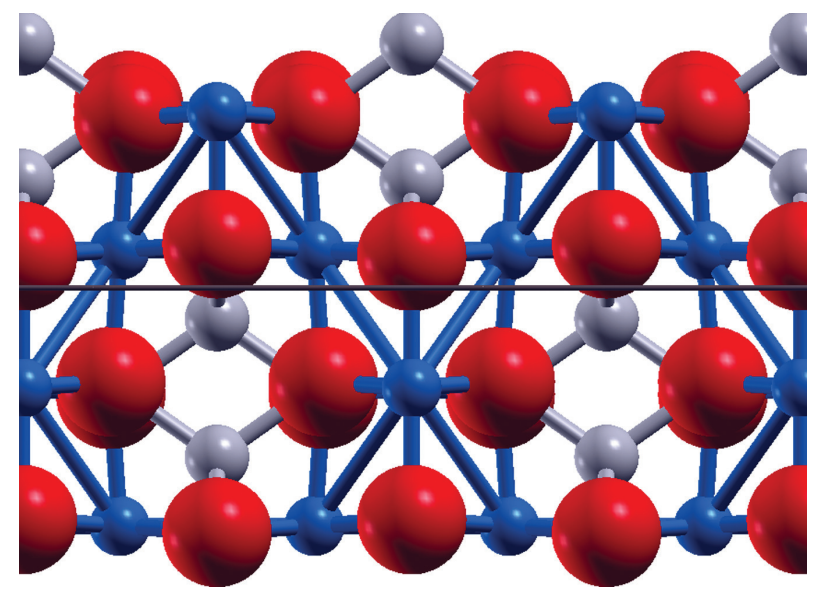

Fig. 11: Unrelaxed, stoichiometric $\mathrm{ZnFe}_{2} \mathrm{O}_{4}$ (100) surface model with 16 atomic layers. $\mathrm{Zn}$ atoms are depicted in gray, Fe atoms in blue and $\mathrm{O}$ is shown in red. The $\mathrm{ZnO}_{2}$ termination is shown in the topmost layer, the $\mathrm{FeO}_{5}$ termination in the bottom layer. 
from both topmost and bottom layer creating a symmetric, stoichiometric surface model. Its top and side view are shown in Figure 12.

By increasing the number of the atomic layers the convergence of the positions of the highest occupied crystal orbital, HOCO, and the lowest unoccupied crystal orbital, LUCO, was tested. Their positions, shown in Figure 13 as a function of the number of surface units, are related to the potential required for photocatalytic water splitting and the band positions for both oxygen evolution reaction, OER, and hydrogen evolution reaction, HER [146-148]. Up-spins are denoted with $\alpha$ and down spins are denoted with $\beta$.

The band gaps of the surface models, in the range of $2.7-2.8 \mathrm{eV}$, are in good agreement with the bulk value, listed in Table 3. HOCO and LUCO of spin-up
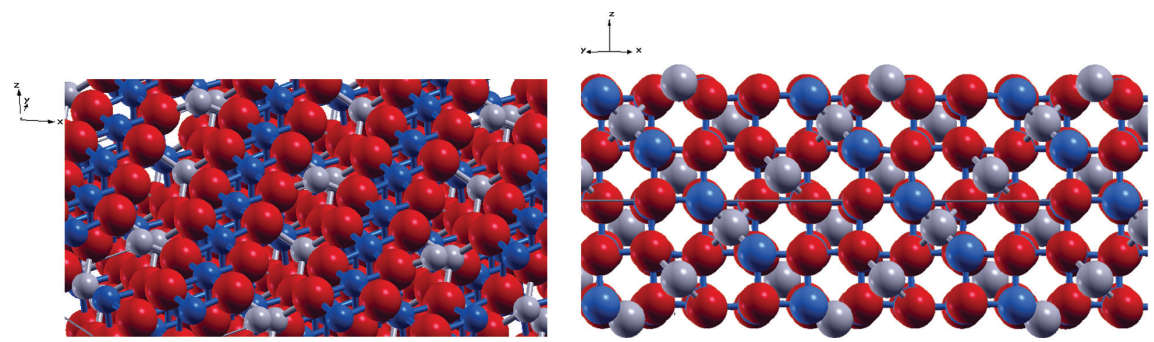

Fig. 12: Top (left) and side (right) view of a 21-layer model of the $\mathrm{ZnFe}_{2} \mathrm{O}_{4}(100)$ surface with $\mathrm{Zn}$ defects in the topmost layer and additional $\mathrm{Zn}$ atoms in the bottom layer. $\mathrm{Zn}$ atoms are depicted in gray, Fe atoms in blue and 0 is shown in red.

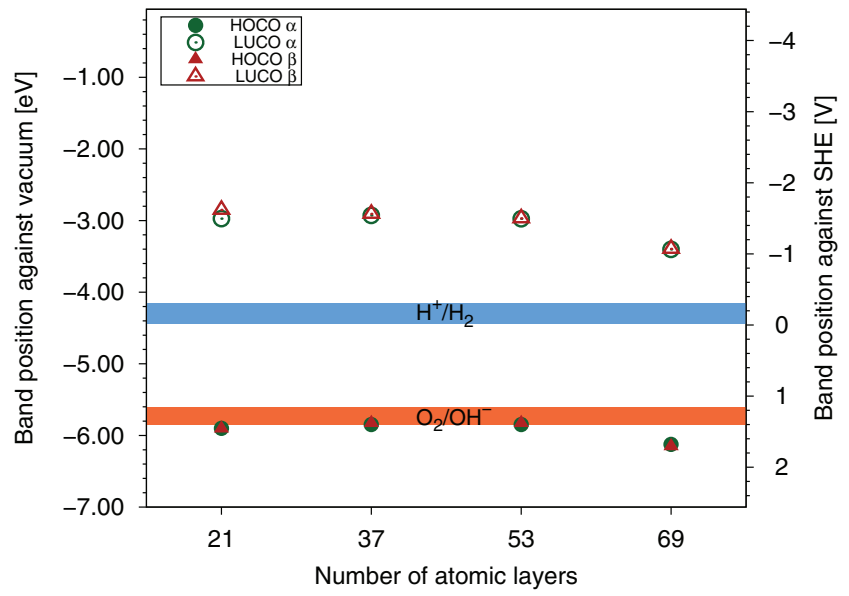

Fig. 13: Absolute HOCO and LUCO energies [eV] of antiferromagnetic defective symmetric stoichiometric surface models of $\mathrm{ZnFe}_{2} \mathrm{O}_{4}$ in dependence of the number of atomic layers in relation to the potentials and overpotentials for OER and HER. 
and spin-down electrons are equal for antiferromagnetic spin ordering. The LUCO positions range between $-3.85 \mathrm{eV}$ and $-3.40 \mathrm{eV}$ against vacuum, respectively $-1.59 \mathrm{~V}$ to $-1.04 \mathrm{~V}$ against SHE. While this is in good agreement with the flat band potential of $\mathrm{p}$-type $\mathrm{ZnFe}_{2} \mathrm{O}_{4}$ found in experimental literature (shown in Figure 22a in Section 3.1.2), it does not match the experimental flat band potentials shown in Figure 22b or flat band potentials found in recent literature, as reviewed in the following. Hufnagel and coworkers report the flat band potential of $\mathrm{ZnFe}_{2} \mathrm{O}_{4}$ as $0.65 \mathrm{~V}$ against RHE [149]. This is in good agreement with the lower range of the flat band potentials shown in Figure 22b. In a very recent study by Henning and co-workers the flat band potential of $\mathrm{ZnFe}_{2} \mathrm{O}_{4}$ is reported as $0.82 \mathrm{eV}$ against $\mathrm{RHE}$ [150], which exceeds the lower end of the measured flat band potentials but ties in well with other data from literature [2, 151-153]. One reason for the discrepancy between our calculated results and the experimental ones found in literature as well as the ones reported in Section 3.4.2 is the lack of an implicit solvent model describing the interactions of the surface with water. Unfortunately, the CRYSTAL code does not provide an implicit solvent model. Influences of spin state and cation inversion on the band positions will be discussed in the following.

As shown above for the bulk ferrites, see Section 3.1.1, the influence of the spin state and the resulting magnetization on the band energies of the surface models was investigated as well. Since the effects on the band positions are similar for every model, the results for a model with 53 atomic layers are depicted in Figure 14.

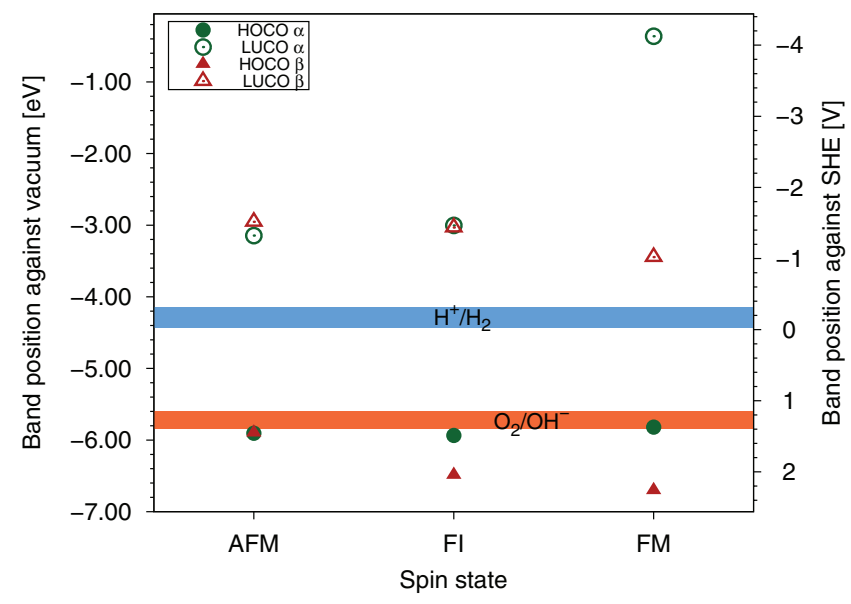

Fig. 14: Absolute HOCO and LUCO energies $(\mathrm{eV})$ of a defective symmetric stoichiometric surface model of the $\mathrm{ZnFe}_{2} \mathrm{O}_{4}$ (100) surface with 53 atomic layers in dependence on the spin state. $\mathrm{AFM}=$ antiferromagnetic, $\mathrm{FM}=$ ferromagnetic, $\mathrm{FI}=$ ferrimagnetic. 
Spin ordering was achieved by defining the magnetization of selected iron atoms, as shown color-coded in Figure 15. FM spin ordering was achieved by attributing an up-spin to every iron atom. For the AFM surface model the symmetry of the spin distribution of the bulk model was conserved. In the chosen slab model there is a central layer of Fe atoms. Attributing these iron atoms with upspins and attributing iron atoms in the outer layers alternatingly with opposing spins leads to ferrimagnetic models. As can be seen from Figure 14, the energy of the up-spin HOCO is barely influenced by the choice of spin configuration. For antiferromagnetic spin ordering, the HOCO energies of up and down-spin are equal and LUCO energies are similar as well. In comparison with the bulk the band gap is slightly lowered to a range of $2.7-2.8 \mathrm{eV}$. Ferrimagnetic spin ordering results in a slightly increased band gap in the up-spin and an increased band gap in the down-spin ladder. The down-spin band gaps of the ferri- and ferromagnetic slab model are almost indistinguishable yet the exact positions differ slightly. In fashion of the FM bulk calculations the up-spin band gap increases to $5 \mathrm{eV}$. Neither ferri- nor ferromagnetic spin ordering leads to a lowering of LUCO energies towards experimental values. The relative stability of the spin states varies with the number of atomic layers used, but energy differences per formula unit are less than $4 \mathrm{~kJ} / \mathrm{mol}$. For the 21-layer model, AFM spin ordering is most stable, for 37 and 69 layers the ferromagnetic state is most stable and for the 53-layer model the most stable spin configuration is ferrimagnetic. Thus, no general trend can be predicted. The small difference in stability underlines the importance of considering different spin configurations when dealing with spinel ferrites. It may also serve as a tentative explanation for the large scattering of reported experimental results for electronic properties, as reported in Section 3.4.2 and 3.1.2.
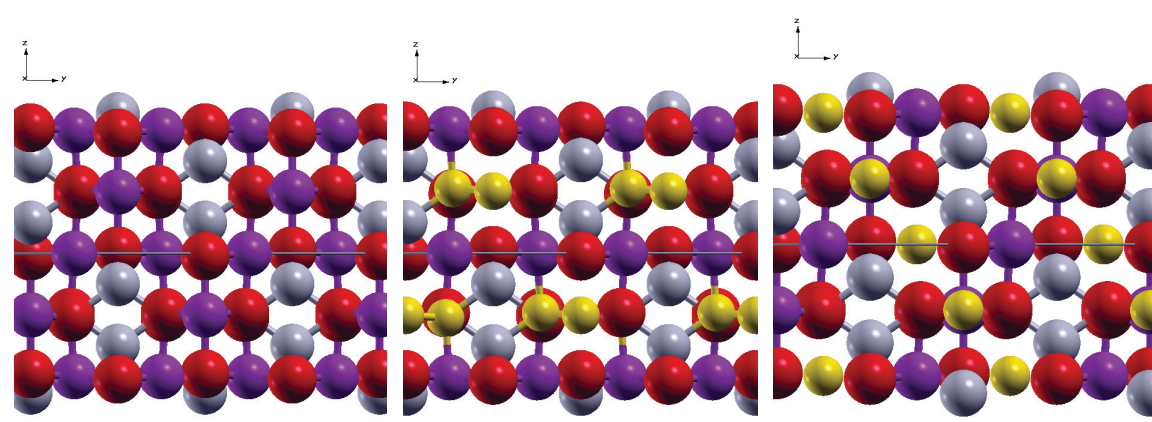

Fig. 15: Ferromagnetic, ferrimagnetic, and antiferromagnetic spin distribution in $\mathrm{ZnFe}_{2} \mathrm{O}_{4}$ (100) surface models. Oxygen atoms are depicted in red, $\mathrm{Zn}$ atoms in gray, up-spin iron atoms are shown in purple and down-spin iron atoms are shown in yellow. 


\subsubsection{Cation exchange and hydroxylation}

After using the DDSCH method to investigate defective surface models, we employed it to investigate the adsorption of $\mathrm{Zn}$-containing species on the polar (100) surface of $\mathrm{ZnFe}_{2} \mathrm{O}_{4}$. Experimental results discussed in Section 3.4.2 hinted that the distribution of zinc and iron atoms can be of importance for the electronic properties of $\mathrm{ZnFe}_{2} \mathrm{O}_{4}$. Additionally, calculations of the bulk material have already proven that the band gap is slightly influenced by the degree of inversion, see Section 3.1.1. We therefore investigated the effect of the $\mathrm{Zn}$ :Fe-exchange for the 21-layer surface model. All of the experiments are performed in aqueous environment. While there are implicit solvation models for a number of program codes, in the CRYSTAL code the aqueous environment has to be modeled by explicit solvation, i.e. the adsorption of water molecules, or depending on the $\mathrm{pH}$ value, hydroxyl species. We investigated a perfect surface by formally adding $\mathrm{Zn}(\mathrm{OH})_{2}$, shown in Figure 12.

For these models the cation distribution of the topmost atomic layers was varied by exchanging Fe ions from the top layer with $\mathrm{Zn}$ atoms from lower bulk-like layers. We varied the $\mathrm{Zn}$ :Fe ratios in the outermost layers of the surface model. The model without cation exchange has a $\mathrm{Zn}$ :Fe ratio of 2:4. This was altered to a $\mathrm{Zn}: \mathrm{Fe}$ ratio of $4: 2$, denoted as $2 \mathrm{Fe}: 4 \mathrm{Zn}$ in Figure 16. In this model iron atoms occupy tetrahedral positions in the lower layers. For the third model, denoted as 0Fe:6Zn in Figure 16, all cation positions in the topmost and bottom layer were occupied with $\mathrm{Zn}$ resulting in pure iron layers in the inner part. The energy difference between these cation distributions is in the same order of magnitude as for

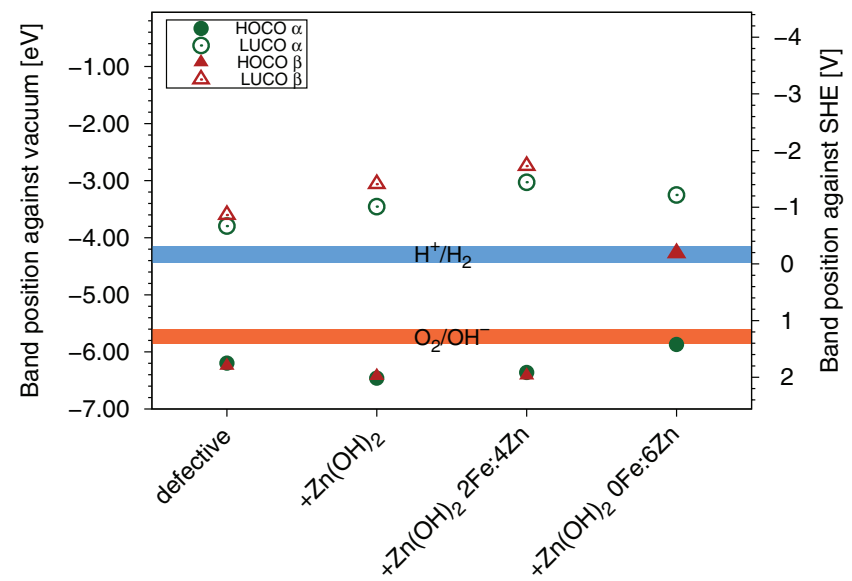

Fig. 16: $\mathrm{HOCO}$ and LUCO positions of an antiferromagnetic $\mathrm{ZnFe}_{2} \mathrm{O}_{4}$ (100) surface model with 21 layers dependent on the cation distribution of the topmost and bottom layer and addatoms. 
the defective models with different spin distributions. Changing the $\mathrm{Zn}$ :Fe ratio of the top and bottom layer from 2:4 to 4:2 leads to an energetically more favorable structure. Increasing the amount of zinc further to layers containing only $\mathrm{Zn}$ or only Fe atoms is energetically less stable than the ideal and half exchanged configurations. The effect of hydroxylation and cation exchange on HOCO and LUCO energies of the antiferromagnetic 21-layer model are displayed in Figure 16. The addition of $\mathrm{Zn}(\mathrm{OH})_{2}$ results in an increase of the band gap and in a larger energetic splitting of up and down-spin orbitals. For a $\mathrm{Zn}$ :Fe ratio of 4:2 in the topmost layer, the band gap increases by $0.5 \mathrm{eV}$ while shifting the up-spin levels upwards by $0.5 \mathrm{eV}$. Changing the $\mathrm{Zn}$ :Fe ratio to $4: 2$ does not affect the HOCO positions but further raises up- and down-spin LUCO positions. Surface layers fully occupied by $\mathrm{Zn}$ atoms lead to a vanishing up-spin band gap and increased down-spin LUCO position as well as a reduced down-spin band gap.

For a more detailed analysis of the electron-hole separation after photoexcitation, the shape of the HOCO and the LUCO was inspected. Up-spin HOCO and LUCO of the hydroxylated model without any zinc enrichment in the terminating layers are shown in Figure 17. Similar to the results for the bulk with normal cation distribution and without oxygen vacancies, shown in Section 3.1.1 Figure 1,
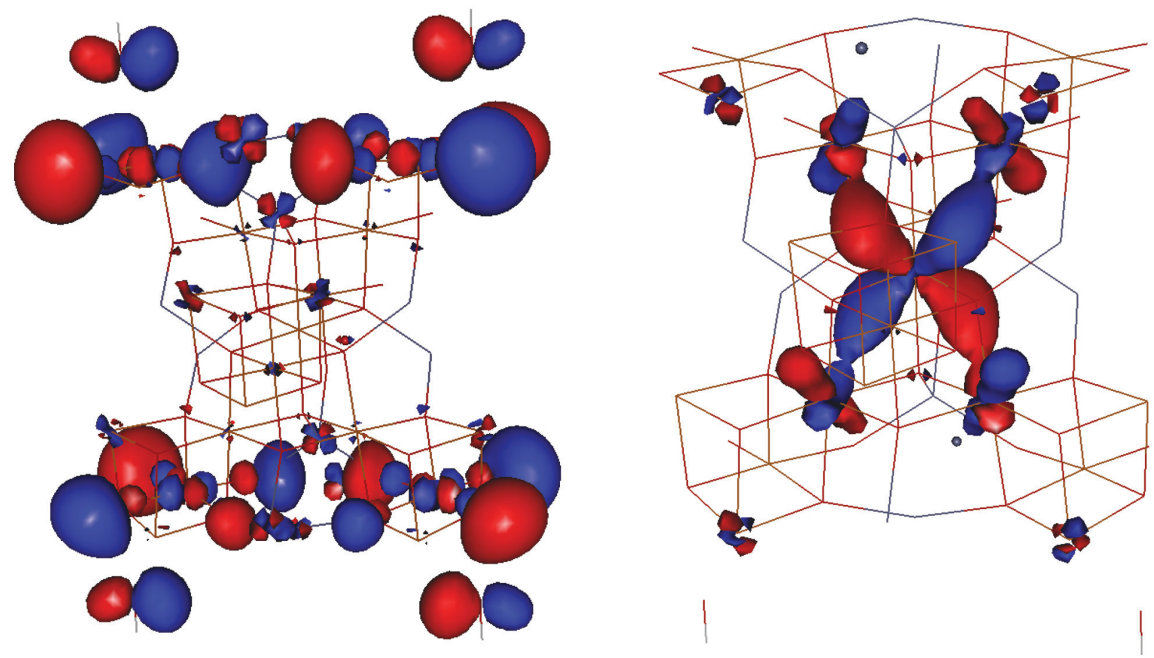

Fig. 17: Up-spin HOCO (left) and LUCO (right) of a 21 layer model of $\mathrm{ZnFe}_{2} \mathrm{O}_{4}$ (100) with the formal addition of $\mathrm{Zn}(\mathrm{OH})_{2}$. 
the HOCO mostly consists of oxygen 2p-orbitals, but is also located on the oxygen atoms of the adsorbed hydroxyl-species. The HOCO is strongly localized on both terminating layers of the model with small contributions in the bulk-like central layers of the slab model. In contrast, the LUCO is located mostly in the inner part of the slab model. Similar to the bulk it consists of Fe 2d-orbitals. Exchanging two of the outer iron atoms with inner zinc atoms leads to a more pronounced delocalization of the HOCO over the entire slab, as seen in Figure 18. In contrast, the LUCO becomes even more localized in the center of the bulk-like layers. Due to the delocalized HOCO and the overlap with the LUCO the electron-hole recombination might be enhanced, decreasing the efficiency of the photocatalyst.

Due to the separation of the cation species in the purely $\mathrm{Zn}$ terminated model, the HOCO, seen on the left in Figure 19, again is mostly localized on the terminating atomic layers. It consists of oxygen 2p-orbitals and zinc 3d-orbitals, however, no contribution of the adsorbed hydroxyl species can be found. The orbitals of the adsorbed hydroxyl species do not contribute to the up-spin LUCO either. Again, the LUCO is localized in the central atomic layers and consists of hybridized iron 3d-orbitals.
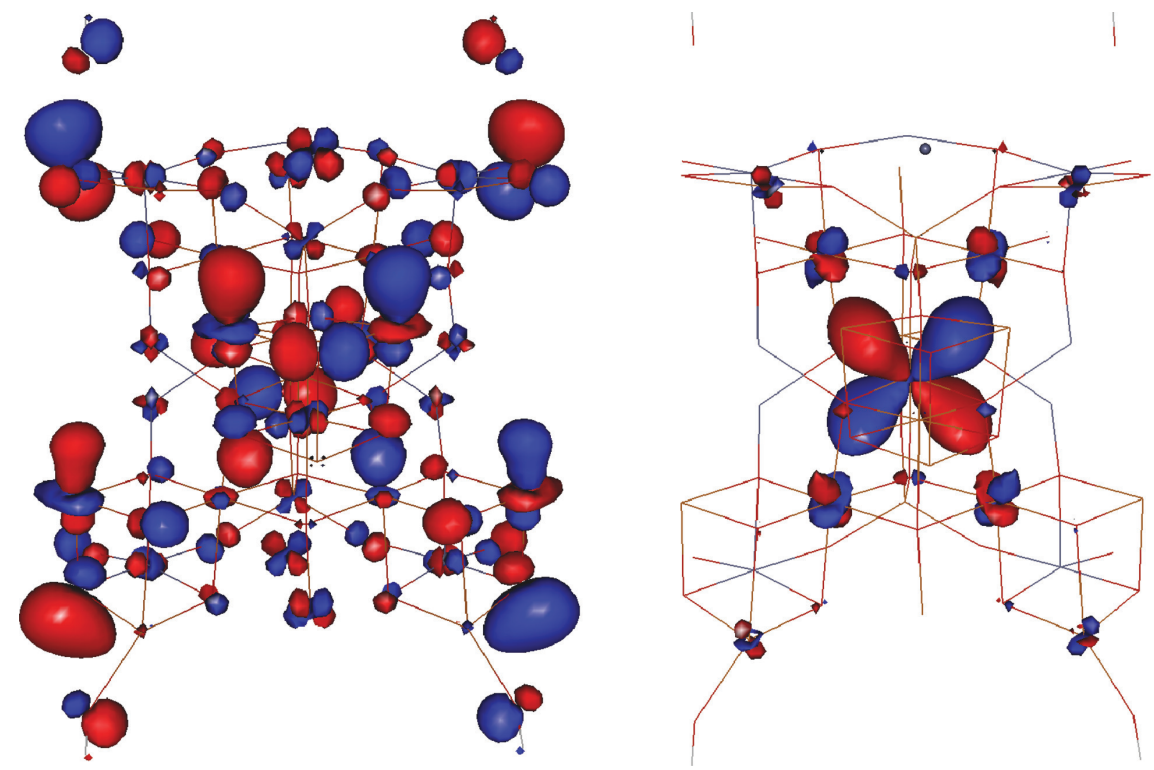

Fig. 18: Up-spin HOCO (left) and LUCO (right) of a 21 layer model of $\mathrm{ZnFe}_{2} \mathrm{O}_{4}$ (100) with the formal addition of $\mathrm{Zn}(\mathrm{OH})_{2}$ and a $\mathrm{Zn}$ :Fe ratio of $4: 2$ in the topmost and bottom layer. 

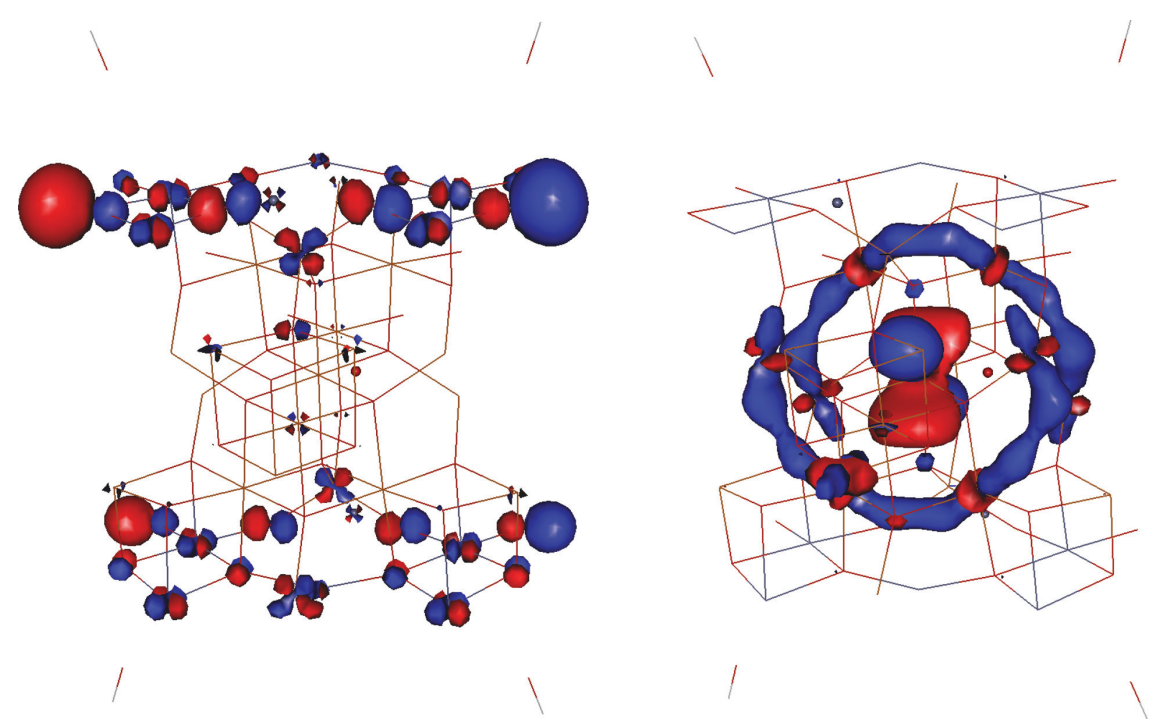

Fig. 19: Up-spin HOCO (left) and LUCO (right) of a 21 layer model of $\mathrm{ZnFe}_{2} \mathrm{O}_{4}$ (100) with the formal addition of $\mathrm{Zn}(\mathrm{OH})_{2}$ and a $\mathrm{Zn}$ :Fe ratio of 6:0 in the topmost and bottom layer.

\subsubsection{Nanotube models}

As introduced in Section 2.3, our third model type to investigate the properties of the polar (100) surface of $\mathrm{ZnFe}_{2} \mathrm{O}_{4}$ are nanotubes. Unlike the defective surface model and the surface model with adsorption the nanotube model exposes both $\mathrm{Zn}$ and Fe surface layers. The inherent dipole moment of the flat surface is canceled by the nanotube's circular symmetry. Previous work on non-polar surfaces has shown that the electronic properties of surfaces and nanotubes do not differ significantly despite the strain caused by the latter's curvature [64].

We therefore exploited the capability of the CRYSTAL code to build nanotubes from surface unit cells as models for the $\mathrm{ZnFe}_{2} \mathrm{O}_{4}$ (100) surface [63]. The automatic generation of a nanotube from a two-dimensional slab model exploits symmetry: First, the desired slab is chosen by its Miller indices and the number of atomic layers as described above. Then the number of surface units rolled up into a nanotube are given. The number of surface units determines the diameter and therefore curvature of the nanotube and has to be converged as well. Rolling up the stoichiometric surface model into a nanotube does not only cancel of the dipole moment but also allows to investigate both terminations. As shown in Figure 20, the outside of the nanotube models in this work is terminated by $\mathrm{FeO}_{5}$ while the inside exposes $\mathrm{ZnO}_{2}$. Results presented for the nanotube models were obtained using a PW1PW [154] optimized bulk and nanotube structure. The bulk was optimized in the configuration denoted as A-afm1 in Chapter 3.1.1 and the spin distribution of the bulk iron atoms was preserved in the nanotube. 


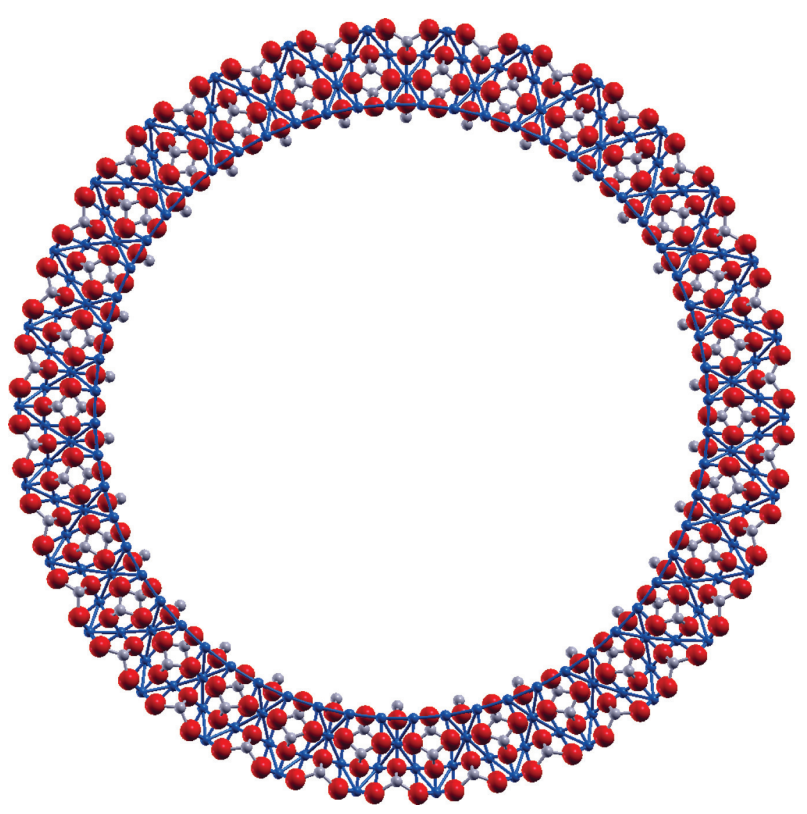

Fig. 20: Unrelaxed nanotube model of $\mathrm{ZnFe}_{2} \mathrm{O}_{4}$ (100) with 16 atomic layers and a diameter of 28.5 $\AA$. $\mathrm{Zn}$ atoms are depicted in gray, Fe atoms are blue and $\mathrm{O}$ is shown in red.

In order to increase SCF convergence and decrease computational costs the geometry of the nanotubes was first relaxed using a GGA functional. This pre-optimized structure was re-optimized with PW1PW. Since the dielectric dependent self consistent hybrid method employed for the previous results was not available in the program version used for the nanotube calculations, the electronic structure was calculated with PW1PW with a reduced Fock exchange of $14 \%$.

This method provides a band gap of $1.9 \mathrm{eV}$ for the bulk which is in good agreement with the lower range of experimental values as can be seen in Table 5 . Band gaps for the nanotube models were quite similar to the bulk, in the range of $1.87-1.99 \mathrm{eV}$ as depicted in Figure 21. In agreement with the results for the defective symmetric stoichiometric surface models discussed in the previous section, this indicates the absence of surface-induced states for $\mathrm{ZnFe}_{2} \mathrm{O}_{4}$ (100). While the $\mathrm{HOCO}$ energies of the nanotube model are quite similar to the ones of models with defects, shown in Figure 13, the smaller amount of Fock exchange leads to a lowering of the LUCO energies. Again, the positions of the highest occupied crystal orbitals, HOCO, of up and down-spin electrons are essentially the same. The positions of the lowest unoccupied crystal orbitals, LUCO, vary only by $0.05 \mathrm{eV}$. With a position of $-4.2 \mathrm{eV}$ against vacuum, respective $0.3 \mathrm{~V}$ against SHE, they are in good agreement with the upper range of measured flat band positions shown in Figure 22b. 


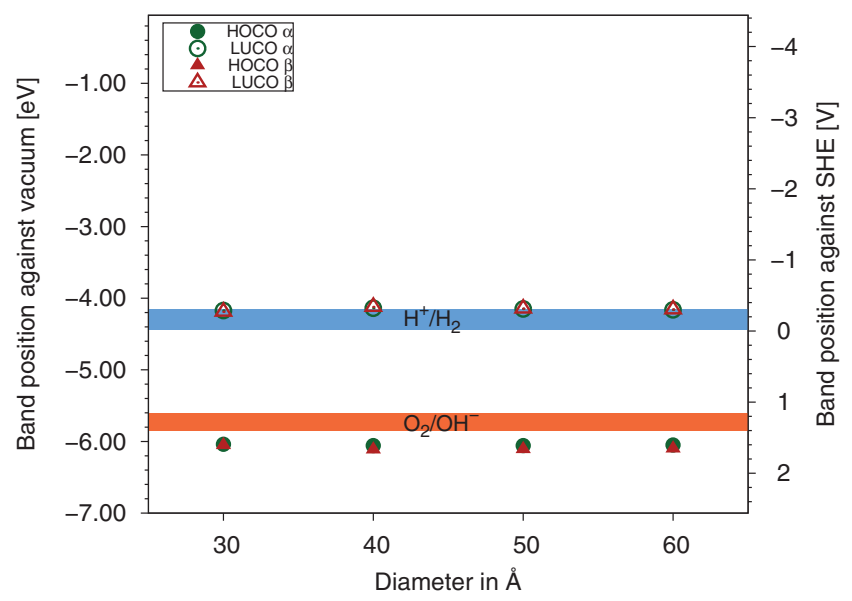

Fig. 21: Band position of $\mathrm{ZnFe}_{2} \mathrm{O}_{4}(100)$ nanotube surface models with 16 atomic layers in dependence on the diameter of the nanotube in relation to the potentials and overpotentials for OER and HER.

This indicates that nanotube models are in principle suitable to study electronic properties of polar surfaces and appear to be more suited to describe the band positions of experimentally prepared $\mathrm{ZnFe}_{2} \mathrm{O}_{4}$ samples. However, the computational cost is comparably high and nanotube surface models with a larger number of atomic layers lead to problems in the SCF procedure in the wavefunction calculation. Furthermore, despite the exploitation of symmetry, the SCF convergence is also dependent on the termination of the nanotube since models with the Zn-terminated surface on the outside could not reach SCF convergence. We therefore did not continue with this type of surface models.

\subsubsection{Experimental results}

Various experimental techniques, such as XPS [155, 156], UPS [157, 158], and electrochemical impedance spectroscopy (EIS) [159] are commonly used for the determination of the absolute band position of $\mathrm{ZnFe}_{2} \mathrm{O}_{4}$. Among these methods, EIS is largely applied because it allows a straightforward determination of the flat band potential, i.e. the Fermi level, of a sample [160]. For highly n-type and ptype doped semiconductors, the flat band potential is located close to the conduction band edge and to the valence band edge, respectively. Therefore, it is generally accepted to consider the energetic band edge position of the conduction band for n-type materials and the valence band for $\mathrm{p}$-type materials to be close to the flat band potential. Once the energetic position of one of the bands is 

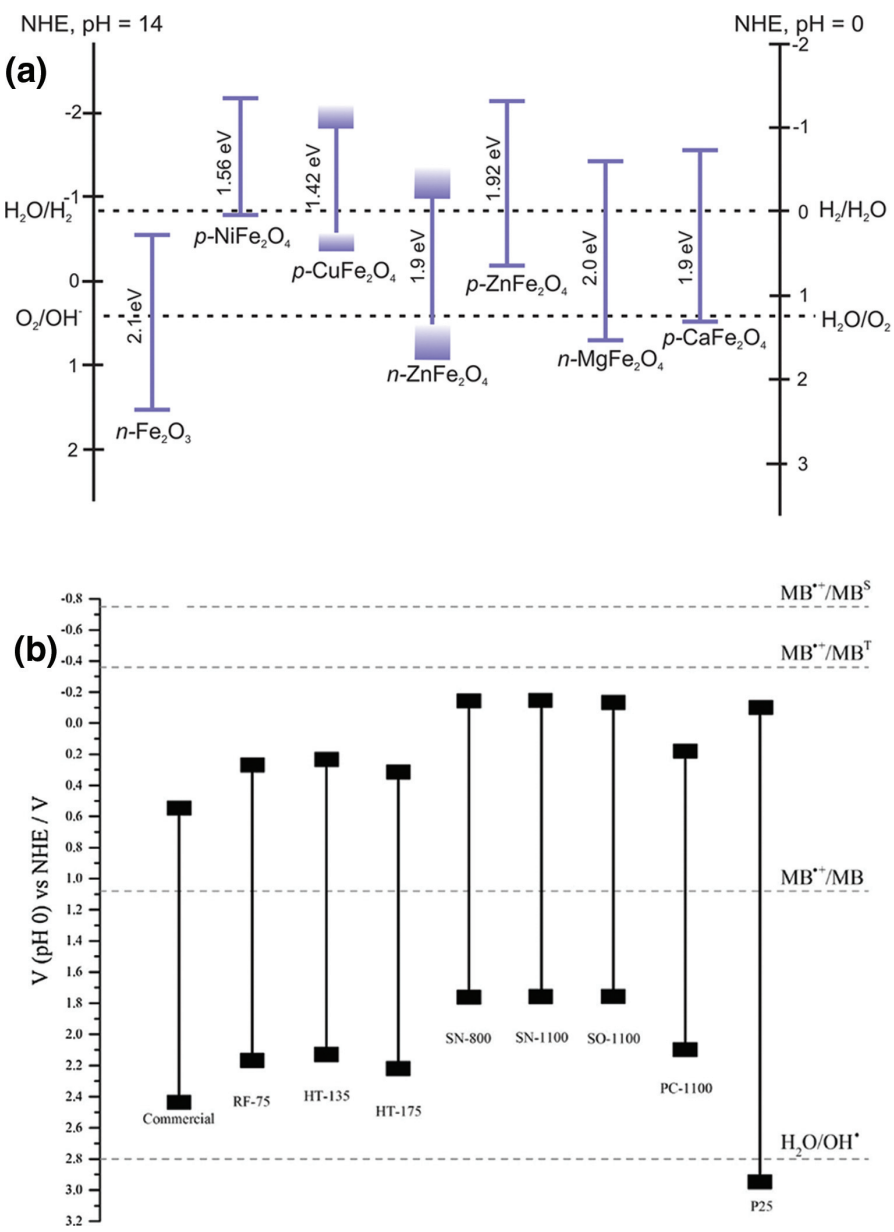

Fig. 22: (a) Experimentally determined band positions of $\mathrm{ZnFe}_{2} \mathrm{O}_{4}$ and other spinel ferrites. Reproduced from reference [2] with permission from AIP Publishing. (b) Band positions of $\mathrm{ZnFe}_{2} \mathrm{O}_{4}$ samples prepared by reflux (RF-75), hydrothermal (HT-135 and HT-175), solid-state reaction (SN-800, SN-1100, and SO-1100), and polymer complex method (PC-1100). Band positions of commercial $\mathrm{ZnFe}_{2} \mathrm{O}_{4}$ and $\mathrm{TiO}_{2}$ samples are shown for comparison. Reproduced from reference [159] with permission from Elsevier.

determined, the position of the other one is immediately deduced considering that the difference between them corresponds to the optical band gap energy of the material [161, 162]. Figure 22a shows the absolute band position of different spinel ferrites as obtained in the literature [2]. The band position of n-type $\mathrm{ZnFe}_{2} \mathrm{O}_{4}$, corresponding to an average of several published values determined mostly by EIS, exhibits a dispersion of approximately $0.5 \mathrm{~V}$ [2]. Figure 22b shows the energetic 
band position determined by EIS for different $\mathrm{ZnFe}_{2} \mathrm{O}_{4}$ samples synthesized by reflux, hydrothermal, solid-state reaction, and polymer complex methods [159]. Large deviations up to $\approx 0.7 \mathrm{~V}$ are observed for this set of samples. It is well known that the degree of inversion and the presence of defect states closely depend on the synthetic conditions [108, 124, 163-167]. Therefore, these properties appear to be responsible for the large experimental deviations observed in the absolute band position between the different $\mathrm{ZnFe}_{2} \mathrm{O}_{4}$ samples. Such an impact of the degree of inversion and the defect states is in agreement with the theoretical predictions presented in the section above. It worth mentioning that semiconductor electrodes commonly used for flat band potential determination via EIS do not meet the ideal conditions on which the theory behind the measurement is based [162]. Perfectly planar interfaces, uniform distribution of donors, and the absence of surface states are some of the conditions that an electrode should meet to obtain a reliable EIS analysis of the flat band potential [162]. Therefore, more careful experimental determinations of the flat band potential should be performed in order to unequivocally confirm the theoretical predictions.

The calculated surface models show that the band gap increases with increasing zinc amount, see Figure 16 . An addition of $\mathrm{Zn}(\mathrm{OH})_{2}$ surface states did increase the band gap by $0.5 \mathrm{eV}$ compared to the same sample without accumulated zinc at the surface. A similar trend could also be observed from the measured optical band gaps for electrodeposited zinc ferrite films with different stoichiometry, but the measured values have to be handled with care because optical band gaps are not identical with the electronic band gap and Tauc-plots are sometimes ambiguous and difficult to interpret. The zinc depleted samples have an approximately 0.2-0.3 eV lower optical band gap compared with the stoichiometric sample as seen in Figure 23. Contrary to the theoretical values the film with zinc enriched surface shows an identical band gap (within measurement error) compared to the stoichiometric film.

For measuring the absolute band positions we performed Mott-Schottky plots (see Figure 24) of the films to estimate the flatband potential $\left(\mathrm{V}_{\mathrm{fb}}\right)$. In practice, the measured flatband potentials are around $100-200 \mathrm{mV}$ below the conduction band [168]. All measured films show $\mathrm{n}$-type semiconductor behavior and the obtained values fairly estimate the bottom of the conduction band edge. Here the limitations of the EIS technique has to be reminded as mentioned beforehand. The used electrodeposited samples with varying stoichiometry do by no means exhibit a planar interface as shown by SEM pictures in our previous work and surface states cannot be excluded [122].

The data in Figure 24 indicate that with increasing zinc amount the flatband potential is shifted to more negative values. The flatband potential for the zinc 


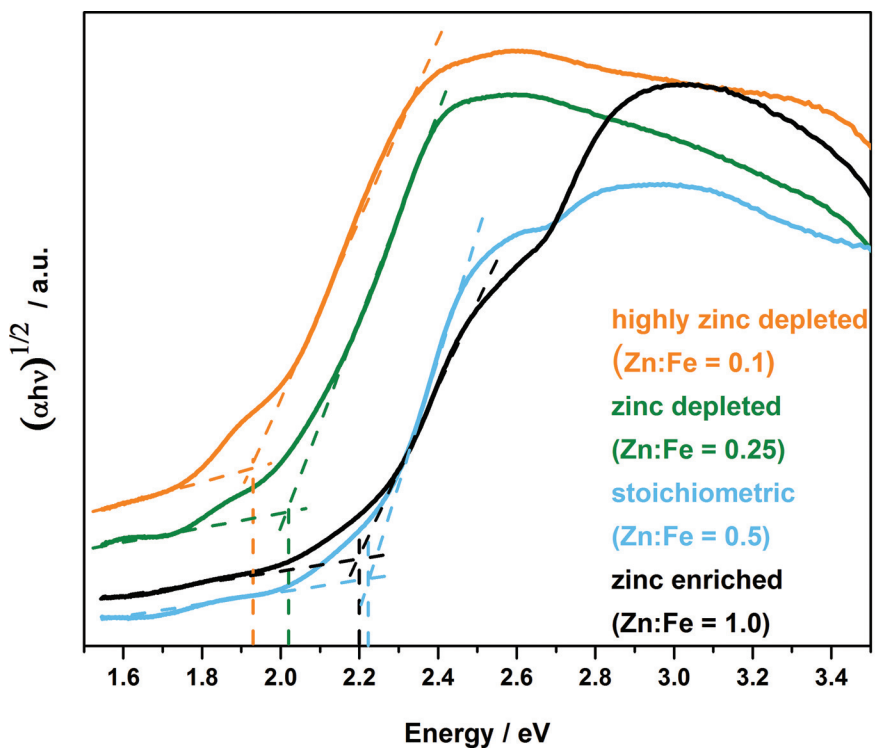

Fig. 23: Tauc-plots estimating indirect optical band gap of zinc ferrite films with varying compositions.

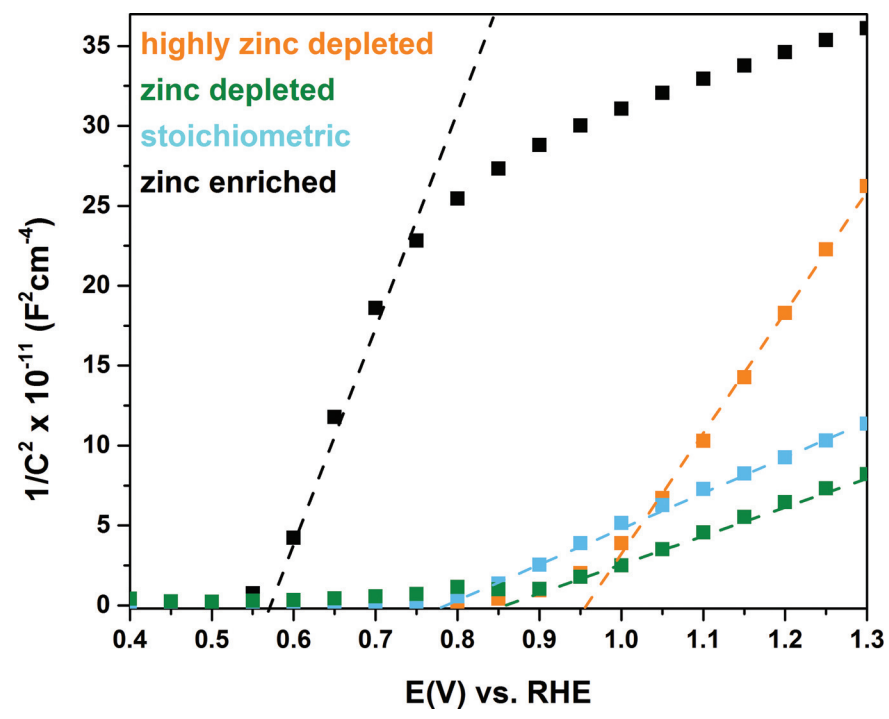

Fig. 24: Mott-Schottky-plots of electrodeposited zinc ferrite films with varying surface stoichiometry. All samples are measured in $1 \mathrm{M} \mathrm{NaOH}$ electrolyte with applied frequency of $10 \mathrm{kHz}$. The color codes and given stoichiometry are described in Figure 6. 
enriched sample is negatively shifted by $150 \mathrm{mV}$ compared to the stoichiometric sample whose flatband potential is located at $+700 \mathrm{mV}$ vs. RHE. The zinc depleted sample is positively shifted by $60 \mathrm{mV}$, for a highly zinc depleted sample the flatband potential is even more positively shifted by $220 \mathrm{mV}$ compared to the stoichiometric sample. The shift of the flatband potential with composition leads to different photocurrent onsets which determine the working point of the photoelectrochemical cells. Despite the varying ratios the data show that all our prepared films are not suited for HER due to the more positive conduction band edge relative to the $\mathrm{H}^{+} / \mathrm{H}_{2}$ reaction.

For oxygen evolution, the valence band of the catalyst has to be located at $1.23 \mathrm{~V}$ vs. RHE or more positive [1]. Assuming that the band gap of the experimental samples is identical with the optical band gap measured by Tauc-plots (Figure 23), the position of the valence band for all our samples are located at $2.9 \mathrm{~V}$ vs. RHE (highly zinc depleted sample), $2.8 \mathrm{~V}$ vs. RHE (zinc depleted sample and stoichiometric sample) and $2.6 \mathrm{~V}$ vs. RHE (zinc enriched sample), respectively. Therefore our zinc ferrite film samples are suitable as oxygen evolving catalyst due to an appropriate position of the valence band, but not for overall water splitting due the mismatch of the conductive band position. This is different from the theoretical predictions for stoichiometric and $\mathrm{Zn}$ enriched surfaces shown in Figures 13, 14,16 , and 21. There the LUCO is always at energies below $-1000 \mathrm{meV}$ vs. RHE.

We have observed that the photoelectrocatalytic activity of electrodeposited zinc ferrite films is strongly dependent on their composition. The theoretical investigations in this work showed that oxygen defect states are responsible for the relatively low optical band gaps. Furthermore, oxygen defects results low photoelectrocatalytic activity by serving as recombination center that will hinder the charge carrier mobility. Zinc enriched samples are expected to exhibit the highest amount of oxygen defect states due to the population of $\mathrm{Zn}^{2+}$ ions in place of $\mathrm{Fe}^{3+}$ ions. Oxygen defects are a necessary result in order to ensure charge neutrality.

The experimental photocurrent transient measurements indeed prove that the zinc enriched sample show very low photocurrents (Figure 25). Compared with samples with other stoichiometries, the zinc enriched one seems to be almost photoelectrocatalytically inactive. The zinc enriched sample $(\mathrm{Zn}: \mathrm{Fe}$ ratio $=0.25)$ shows an improved photocurrent compared to the stoichiometric sample and exhibits the best photocurrent of all measured samples. However, the highly depleted zinc sample $(\mathrm{Zn}: \mathrm{Fe}$ ratio $=0.1)$ exhibits a lower photocurrent compared with the stoichiometric sample. Here the zinc depleted sample is analogous to a sample with higher degree of inversion which does improve the photocurrent [107].

The highly zinc depleted sample is no longer a single phase, since it contains hematite phase impurities, which has a deteriorating effect on the photocurrent. 


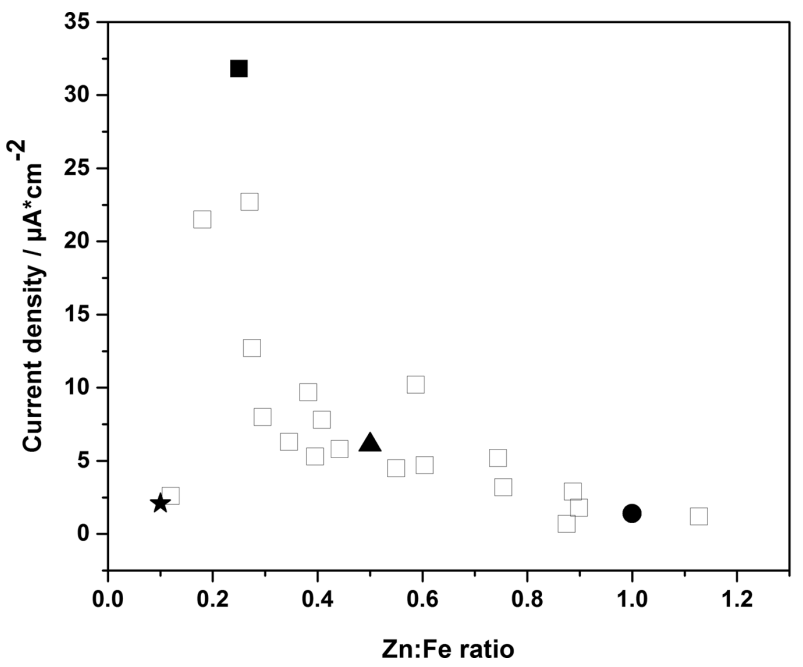

Fig. 25: Current density against composition of electrodeposited zinc ferrite samples with various $\mathrm{Zn}$ :Fe ratios measured by XPS. All data is measured with an applied potential of $1.45 \mathrm{~V}$ vs. RHE in $1 \mathrm{M} \mathrm{NaOH}$ electrolyte and $100 \mathrm{~mW} / \mathrm{cm}^{2}$ white light illumination. Solid symbols indicate the samples as mentioned in this work (circle $=$ zinc enriched $(\mathrm{Zn}:$ Fe ratio $=1.0)$, triangle $=$ stoichiometric $(\mathrm{Zn}: \mathrm{Fe}$ ratio $=0.5)$, square $=$ zinc depleted $(\mathrm{Zn}:$ Fe ratio $=0.25)$, asterisk $=$ highly zinc depleted $(\mathrm{Zn}: F e$ ratio $=0.1)$. Data is adapted from reference [122].

The work by Kimmich et al. [127] did also show that a high amount of hematite impurities has a negative effect on the photoelectrocurrent of zinc ferrite samples. Furthermore, not only the magnitude of the photocurrent but also the photocurrent onset is influenced by the composition of the films. In our study we observed that zinc depleted samples have delayed onsets while zinc enriched samples show earlier photocurrent onsets (determined from chopped light voltammetry) in agreement with the flatband potential measurements [122].

\section{Conclusion}

In this study, we evaluated ferrites $M \mathrm{Fe}_{2} \mathrm{O}_{4}$ with $M=\mathrm{Mg}$, and the first row subgroup elements Sc to $\mathrm{Zn}$ as potential catalyst materials for photoelectrochemical water splitting. By performing dielectric dependent self-consistent hybrid DFT calculations, we were able to show that cation distribution and spin state crucially influence the electronic properties. For most of the investigated ferrites, several spin and cation configurations are energetically close. This result is an indication that the high variety of experimental results originates in the presence of different cation distributions or spin states. Evaluating the results for the novel compounds 
with $M=\mathrm{Sc}-\mathrm{Cr}$, only $\mathrm{ScFe}_{2} \mathrm{O}_{4}$ and $\mathrm{CrFe}_{2} \mathrm{O}_{4}$ are considered as possible candidates for photoelectrochemical water splitting catalysts.

Our calculations for $\mathrm{ZnFe}_{2} \mathrm{O}_{4}$ models with oxygen defects show, that in the presence of oxygen vacancies the band gap energy is significantly reduced. This is in agreement with experimental studies of the oxygen partial pressure dependence of the band gap [100]. To evaluate the influence of inversion on the Raman spectrum of $\mathrm{ZnFe}_{2} \mathrm{O}_{4}$ we calculated Raman band positions and intensities. Due to a substantial shift of some Raman peaks upon inversion of $\mathrm{ZnFe}_{2} \mathrm{O}_{4}$, we conclude that Raman spectroscopy can be used to determine whether inversion is present in the material.

To further investigate the influence of cation distribution and spin state on the properties of $\mathrm{ZnFe}_{2} \mathrm{O}_{4}$, we calculated optical spectra using a $G W$-BSE approach. Upon inversion and by changing the configuration to a ferromagnetic state, the intensity of the first maximum is decreased. This is an indication that inverse and ferromagnetic phases of $\mathrm{ZnFe}_{2} \mathrm{O}_{4}$ downgrade the catalytic performance of the material. It has been shown both by calculation of absolute band positions and flatband measurements that the most stable (100) surface of $\mathrm{ZnFe}_{2} \mathrm{O}_{4}$ is not suited for photocatalytic water splitting. However, the reasons are still not fully clear, since there is a discrepancy between the absolute HOCO and LUCO energies determined theoretically and experimentally. This has to be addressed in the future. Both, spin distribution and cation distribution have a significant influence on the absolute positions of HOCO and LUCO. Nanotubes present a possible way of modeling polar surfaces, however they also suffer from SCF convergence problems with a large number of atoms.

In general, a good agreement between the theoretical predictions and experimental results is observed for the electronic and optical properties of spinel ferrites. Thus, theoretical calculations are a powerful tool for achieving a fundamental understanding of the physicochemical properties of ferrites and how they are affected by the cation inversion and deviations from ideal stoichiometry.

Acknowledgment: We gratefully acknowledge financial support by the Deutsche Forschungsgemeinschaft within the priority program SPP 1613 'Fuels Produced Regeneratively Through Light-Driven Water Splitting: Clarification of the Elemental Processes Involved and Prospects for Implementation in Technological Concepts' (BR 1768/9-1, BA 1137/22-1, WA 1116/28). The authors A. Ulpe, K. C. L. Bauerfeind and T. Bredow thank the Paderborn Center for Parallel Computing, PC2, and the Leibniz University IT Services (former Regionales Rechenzentrum für Niedersachsen [RRZN]) for providing computational resources. A. Ulpe thankfully acknowledges the financial support by the International Max 
Planck Research School on Reactive Structure Analysis for Chemical Reactions (IMPRS-RECHARGE).

\section{References}

1. R. Dillert, D. H. Taffa, M. Wark, T. Bredow, D. W. Bahnemann, APL Mater. 3 (2015) 104001.

2. D. H. Taffa, R. Dillert, A. C. Ulpe, K. C. L. Bauerfeind, T. Bredow, D. W. Bahnemann, M. Wark, J. Photon. Energy 7 (2017) 12009.

3. V. N. Antonov, B. N. Harmon, Phys. Rev. B 67 (2003) 024417.

4. I. Leonov, A. N. Yaresko, V. N. Antonov, M. A. Korotin, V. I. Anisimov, Phys. Rev. Lett. 93 (2004) 146404.

5. H.-T. Jeng, G. Y. Guo, D. J. Huang, Phys. Rev. B 74 (2006) 195115.

6. P. Piekarz, K. Parlinski, A. M. Oleś, Phys. Rev. B 76 (2007) 165124.

7. C. Cheng, C.-S. Liu, J. Phys.: Conf. Ser. 145 (2009) 012028.

8. M. Feng, A. Yang, X. Zuo, C. Vittoria, V. G. Harris, J. Appl. Phys. 107 (2010) 09A521.

9. D. Fritsch, C. Ederer, Phys. Rev. B 82 (2010) 104117.

10. Y. H. Hou, Y. J. Zhao, Z. W. Liu, H. Y. Yu, X. C. Zhong, W. Q. Qiu, D. C. Zeng, L. S. Wen, J. Phys. D: Appl. Phys. 43 (2010) 445003.

11. D. M. Wells, J. Cheng, D. E. Ellis, B. W. Wessels, Phys. Rev. B 81 (2010) 174422.

12. M. Feng, X. Zuo, C. Vittoria, V. G. Harris, J. Wu, IEEE Trans. Magn. 47 (2011) 324.

13. Z. Jiang, W. Zhang, W. Shangguan, X. Wu, Y. Teraoka, J. Phys. Chem. C 115 (2011) 13035.

14. M. S. Senn, I. Loa, J. P. Wright, J. P. Attfield, Phys. Rev. B 85 (2012) 125119.

15. Q.-C. Sun, H. Sims, D. Mazumdar, J. X. Ma, B. S. Holinsworth, K. R. O’Neal, G. Kim, W. H. Butler, A. Gupta, J. L. Musfeldt, Phys. Rev. B 86 (2012) 205106.

16. X. Yu, S.-G. Wang, Y.-W. Li, J. Wang, H. Jiao, J. Phys. Chem. C 116 (2012) 10632.

17. D. Fritsch, C. Ederer, Phys. Rev. B 86 (2012) 014406.

18. D. A. Andersson, C. R. Stanek, Phys. Chem. Chem. Phys. 15 (2013) 15550.

19. A. Elfalaky, S. Soliman, J. Alloys Cmpd. 580 (2013) 401.

20. J.-R. Huang, C. Cheng, J. Appl. Phys. 113 (2013) 033912.

21. D. H. Ji, G. D. Tang, Z. Z. Li, X. Hou, Q. J. Han, W. H. Qi, S. R. Liu, R. R. Bian, J. Magn. Magn. Mater. 326 (2013) 197.

22. P. V. Kumar, M. P. Short, S. Yip, B. Yildiz, J. C. Grossman, J. Phys. Chem. C 117 (2013) 5678.

23. J. Noh, O. I. Osman, S. G. Aziz, P. Winget, J.-L. Brédas, Sci. Technol. Adv. Mater. 15 (2014) 044202.

24. D. Odkhuu, P. Taivansaikhan, W. S. Yun, S. C. Hong, J. Appl. Phys. 115 (2014) 17 A916.

25. Z. Rák, C. J. O’Brien, D. W. Brenner, J. Nucl. Mater. 452 (2014) 446.

26. X. Shi, Y.-F. Li, S. L. Bernasek, A. Selloni, Surf. Sci. 640 (2015) 75.

27. C. L. Muhich, V. J. Aston, R. M. Trottier, A. W. Weimer, C. B. Musgrave, Chem. Mater. 28 (2016) 214.

28. S. Liu, D. Xiang, Y. Xu, Z. Sun, Y. Cao, Appl. Energy 202 (2017) 550.

29. D. Fritsch, J. Phys.: Condens. Matter 30 (2018) 095502.

30. H. Liu, G. Seifert, C. Di Valentin, J. Chem. Phys. 150 (2019) 094703. 
31. A. D. Rowan, C. H. Patterson, Phys. Rev. B 79 (2009) 205103.

32. H. M. Widatallah, E. A. Moore, A. A. Babo, M. S. Al-Barwani, M. Elzain, Mater. Res. Bull. 47 (2012) 33995.

33. V. Ziaei, T. Bredow, Eur. Phys. J. B 90 (2017) 29.

34. P. Hohenberg, W. Kohn, Phs. Rev. 136 (1964) B864.

35. W. Kohn, L. J. Sham, Phys. Rev. 140 (1965) A1133.

36. J. P. Perdew, Y. Wang, Phys. Rev. B 45 (1992) 13244.

37. E. J. Baerends, O. V. Gritsenko, R. van Meer, Phys. Chem. Chem. Phys. 15 (2013) 16408.

38. D. Ganyushin, F. Neese, J. Chem. Phys. 138 (2013) 104113.

39. V. I. Anisimov, J. Zaanen, O. K. Andersen, Phys. Rev. B 44 (1991) 943.

40. A. I. Liechtenstein, V. I. Anisimov, J. Zaanen, Phys. Rev. B 52 (1995) R5467.

41. V. I. Anisimov, F. Aryasetiawan, A. I. Lichtenstein, J. Phys.: Condens. Matter 9 (1997) 767.

42. M. Cococcioni, S. de Gironcoli, Phys. Rev. B 71 (2005) 035105.

43. A. D. Becke, J. Chem. Phys. 98 (1993) 1372.

44. A. D. Becke, J. Chem. Phys. 104 (1996) 1040.

45. J. Heyd, G. E. Scuseria, J. Chem. Phys. 121 (2004) 1187.

46. T. M. Henderson, A. F. Izmaylov, G. E. Scuseria, A. Savin, J. Chem. Theory Comput. 4 (2008) 1254.

47. M. A. L. Marques, J. Vidal, M. J. T. Oliveira, L. Reining, S. Botti, Phys. Rev. B 83 (2011) 035119.

48. A. Erba, J. Phys.: Condens. Matter 29 (2017) 314001.

49. F. Aryasetiawan, O. Gunnarsson, Rep. Prog. Phys. 61 (1998) 237.

50. M. Shishkin, G. Kresse, Phys. Rev. B 74 (2006) 035101.

51. M. Rohlfing, S. G. Louie, Phys. Rev. B 62 (2000) 4927.

52. S. Albrecht, L. Reining, R. Del Sole, G. Onida, Phys. Rev. Lett. 80 (1998) 4510.

53. A. Georges, G. Kotliar, Phys. Rev. B 45 (1992) 6479.

54. A. Georges, G. Kotliar, W. Krauth, M. J. Rozenberg, Rev. Mod. Phys. 68 (1996) 13.

55. D. Vollhardt, K. Byczuk, M. Kollar, Dynamical Mean Field Theory. arXiv, :1109.4833:1-31 (2011).

56. F. Pascale, C. M. Zicovich-Wilson, F. López Gejo, B. Civalleri, R. Orlando, R. Dovesi, J. Comput. Chem. 25 (2004) 888.

57. L. Maschio, B. Kirtman, R. Orlando, M. Rèrat, J. Chem. Phys. 137 (2012) 204113.

58. L. Maschio, B. Kirtman, M. Rérat, R. Orlando, R. Dovesi, J. Chem. Phys. 139 (2013) 164101.

59. R. Dovesi, B. Civalleri, C. Roetti, V. R. Saunders, R. Orlando, Rev. Comp. Chem. 21 (2005) 1.

60. P. W. Tasker, Philos. Mag. A 39 (1979) 119.

61. J. Goniakowski, F. Finocchi, C. Noguera, Rep. Prog. Phys. 71 (2007) 016501.

62. C. Noguera, J. Phys.: Condens. Matter 12 (2000) R367.

63. P. D’Arco, Y. Noel, R. Demichelis, R. Dovesi, J. Chem. Phys. 131 (2009) 204701.

64. M.-C. Runkel, O. Wittich, A. Feldhoff, M. Wark, T. Bredow, J. Phys. Chem. C 119 (2015) 5048.

65. C. J. O’Brien, Z. Rák, D. W. Brenner, J. Phys.: Condens. Matter 25 (2013) 445008.

66. A. C. Ulpe, K. C. L. Bauerfeind, T. Bredow, ACS Omega 4 (2019) 4138.

67. D. V. Oliveira, J. Laun, M. F. Peintinger, T. Bredow, J. Comput. Chem. submitted (2019).

68. L. Chiodo, J. M. Carcía-Lastra, A. Iacomino, S. Ossicini, J. Zhao, H. Petek, A. Rubio, Phys. Rev. B 82 (2010) 045207. 
69. R. Tholkappiyan, K. Vishista, Mater. Sci. Semicond. Process. 40 (2015) 631.

70. V. K. Tripathi, R. Nagarajan, Adv. Powder Technol. 27 (2016) 1251.

71. H. G. Kim, P. H. Borse, J. S. Jang, E. D Jeong, O.-S. Jung, Y. J. Suh, J. S. Lee, Chem. Commun. (2009) 5889. DOI: 10.1039/B911805E.

72. A. G. Abraham, A. Manikandan, E. Manikandan, S. Vadivel, S. K. Jaganathan, A. Baykal, P. S. Renganathan, J. Magn. Magn. Mater. 452 (2018) 380.

73. S. K. Durrani, S. Naz, M Mehmood, M. Nadeem, M. Siddique, J. Saudi Chem. Soc. 21 (2017) 899.

74. K. Kirchberg, A. Becker, A. Bloesser, T. Weller, J. Timm, C. Suchomski, R. Marschall, J. Phys. Chem. C 121 (2017) 27126.

75. G. Vasuki, T. Balu, Mater. Res. Expr. 5 (2018) 065001.

76. M. Y. Rafique, P. Li-Qing, Q. Javed, M. Z. Iqbal, Q. Hong-Mei, M. H. Farooq, G. Zhen-Gang, M. Tanveer, Chin. Phys. B 22 (2013) 107101.

77. P. Guo, G. Zhang, J. Yu, H. Li, X. S. Zhao, Colloids Surf. A 395 (2012) 168.

78. A. Silambarasu, A. Manikandan, K. Balakrishnan, S. K. Jaganathan, E. Manikandan, J. S. Aanand, J. Nanosci. Nanotech. 17 (2017) 1.

79. M. Aghazadeh, I. Karimzadeh, M. R. Ganjali, A. Behzad, J. Mater. Sci.: Mater. Electron. 28 (2017) 18121.

80. H. El Ghandoor, H. M. Zidan, M. M. H. Khalil, M. I. M. Ismail, Int. J. Electrochem. Sci. 7 (2012) 5734.

81. P. Chand, S. Vaish, P. Kumar, Physica B 524 (2017) 53.

82. B. S. Holinsworth, D. Mazumdar, Q.-C. Sun H. Sims, M. K. Yurtisigi, S. K. Sarker, A. Gupta, W. H. Butler, J. L. Musfeldt, Appl. Phys. Lett. 103 (2013) 082406.

83. K. Dileep, B. Loukya, N. Pachauri, A. Gupta, R. Datta, J. Appl. Phys. 116 (2014) 103505.

84. N. Helaili, G. Mitran, I Popescu, K. Bachari, I.-C. Marcu, A. Boudjemaa, J. Electroanal. Chem. 742 (2015) 47.

85. A. Lassoued, M. Ben Hassine, F. Karolak, B. Dkhil, S. Ammar, A. Gadri, J. Mater. Sci.: Mater. Electron. 28 (2017) 18857.

86. D. Sharma, N. Khare, Appl. Phys. Lett. 105 (2014) 032404.

87. C. Himcinschi, I. Vrejoiu, G. Salvan, M. Fronk, A. Talkenberger, D. Zahn, D. Rafaja, J. Kortus, J. Appl. Phys. 113 (2013) 084101.

88. A. V. Ravindra, P. Padhan, W. Prellier, Appl. Phys. Lett. 101 (2012) 161902.

89. G. Rekhila, Y. Bessekhouad, M. Trari, Int. J. Hydrogen Energy 38 (2013) 6335.

90. K. C. B. Naidu, W. Madhuri, Bull. Mater. Sci. 40 (2017) 417.

91. A. Lassoued, M. S. Lassoued, F. Karolak, S. García-Granda, B. Dkhil, S. Ammar, A. Gadri, J. Mater. Sci.: Mater. Electron. 28 (2017) 18480.

92. C. Barathiraja, A. Manikandan, A. M. Uduman Mohideen, S. Jayasree, S. Arul Antony, J. Supercond. Nov. Magn. 29 (2016) 477.

93. S. Balaji, R. Kalai Selvan, L. John Berchmans, S. Angappan, K. Subramanian, C. O. Augustin, Mater. Sci. Eng.: B 119 (2005) 119.

94. S. N. Dolia, R. Sharma, M. P. Sharma, N. S. Saxena, Indian J. Pure Appl. Phys. 44 (2006) 774.

95. J. Haetge, C. Suchomski, T. Brezesinski, Inorg. Chem. 49 (2010) 11619.

96. A. Kezzim, N. Nasrallah, A. Abdi, M. Trari, Energy Convers. Manage. 52 (2011) 2800.

97. N. Helaili, Y. Bessekhouad, K. Bachari, M. Trari, Mater. Chem. Phys. 148 (2014) 734.

98. R. Dom, R. Subasri, N. Y. Hebalkar, A. S. Chary, P. H. Borse, RSC Adv. 2 (2012) 12782. 
99. M. A. Valenzuela, P. Bosch, J. Jiménez-Becerrill, O. Quiroz, A. I. Páez, J. Photochem. Photobiol. A: Chem. 148 (2002) 177.

100. M. Sultan, R. Singh, J. Phys. D: Appl. Phys. 42 (2009) 115306.

101. T. Böntgen, K. Brachwitz, R. Schmidt-Grund, M. Lorenz, M. Grundmann, J. Appl. Phys. 113 (2013) 073503.

102. B. Choudhury, A. Choudhury, Physica E 56 (2014) 364.

103. N. Sivakumar, A. Narayanasamy, C. N. Chinnasamy, B. Jeyadevan, J. Phys. Condens. Matter 19 (2007) 386201.

104. S. D. Shenoy, P. A. Joy, M. R. Anantharaman, J. Magn. Magn. Mater. 269 (2004) 217.

105. L. I. Granone, R. Dillert, P. Heitjans, D. W. Bahnemann, ChemistrySelect 4 (2019) 1232.

106. N. Satoh, T. Nakashima, K. Kamikura, K. Yamamoto, Nat. Nanotechnol. 3 (2008) 106.

107. X. Zhu, N. Guijarro, Y. Liu, P. Schouwink, R. A. Wells, F. Le Formal, S. Sun, C. Gao, K. Sivula, Adv. Mater. 30 (2018) 1801612.

108. L. I. Granone, A. C. Ulpe, L. Robben, S. Klimke, M. Jahns, F. Renz, T. M. Gesing, T. Bredow, R. Dillert, D. W. Bahnemann, Phys. Chem. Chem. Phys. 20 (2018) 28267.

109. N. Guijarro, P. Bornoz, M. Prévot, X. Yu, X. Zhu, M. Johnson, X. Jeanbourquin, F. Le Formal, K. Sivula, Sustain. Energy Fuels 2 (2017) 103.

110. P. Chandramohand, M. P. Srinivasan, S. Velmurugan, S. V. Narasimhan, Solid State Chem. 184 (2011) 89.

111. Z. Cvejic, S. Rakic, A. Kremenovic, B. Antic, C. Jovalekic, P. Colomban, Solid State Sci. 8 (2006) 908.

112. T. Yu, Z. X. Shen, Y. Shi, J. Ding, J. Phys. Condens. Matter 14 (2002) L613.

113. S. W. da Silva, F. Nakagomi, M. S. Silva, A. Franco Jr, V. K. Garg, A. C. Oliveira, P. C. Morais, J. Nanoparticle Res. 14 (2012) 798.

114. W. B. White, B. A. DeAngelis, Spectrochim. Acta 23A (1967) 985.

115. P. R. Graves, C. Johnston, J. J. Campaniello, Mater. Res. Bull. 23 (1988) 1651.

116. J. C. Howard, J. D. Enyard, G. S. Tschumper, J. Chem. Phys. 143 (2015) 214103.

117. A. P. Scott, L. Radom, J. Phys. Chem. 100 (1996) 16502.

118. P. Galinetto, B. Albini, M. Bini, M. C. Mozzati, Chapter 11: Raman Spectroscopy in Zinc Ferrites Nanoparticles. In Raman Spectroscopy, IntechOpen (2018), P. 223-251.

119. M. Bini, C. Tondo, D. Capsoni, M. C. Mozzati, B. Albini, P. Galinetto, Mater. Chem. Phys. 204 (2018) 72.

120. P. Dolcet, K. Kirchberg, A. Antonello, C. Suchomski, R. Marschall, S. Diodati, R. Mu nos Espí, K. Landfester, S. Gross, Inorg. Chem. Front. 6 (2019) 1527.

121. D. Makovec, M. Drofenik, J. Nanoparticle Res. 10 (2008) 131.

122. S. Warfsmann, D. H. Taffa, M. Wark, J. Photochem. Photobiol. A: Chem. 362 (2018) 49.

123. R. M. Cornell, U. Schwertmann, The Iron Oxides: Structure, Properties, Reactions, Occurences and Uses, 2 ed., WILEY-VCH, Darmstadt (2004).

124. H. S. C. O’Neill, Eur. J. Miner. 4 (1992) 572.

125. Z. W. Wang, D. Schiferl, Y. S. Zhao, H. S. C. O’Neill, J. Phys. Chem. Solids 64 (2003) 2517.

126. M. Rivero, A. del Campo, A. Mayoral, E. Mazario, J. Sanchez-Marcos, A. Munoz-Bonilla, RSC Adv. 6 (2016) 40067.

127. D. Kimmich, D. H. Taffa, C. Dosche, M. Wark, G. Wittstock, Electrochim. Acta 259 (2018) 204.

128. D. L. A. de Faria, S. V. Silva, M. T. de Oliveira, J. Raman Spectrosc. 28 (1997) 873.

129. P. A. Fernandes, P. M. P. Salomé, A. F. da Cunha, J. Alloys Compd. 509 (2011) 7600. 
130. K. Takanabe, ACS Catal. 7 (2017) 8006.

131. W. Shockley, H. J. Queisser, J. Appl. Phys. 32 (1961) 510.

132. J. W. Verhoeven, Pure Appl. Chem. 68 (1996) 2223.

133. N. Pailhé, A. Wattiaux, M. Gaudon, A. Demourgues, J. Solid State Chem. 181 (2008) 1040.

134. G. Kresse, J. Furthmüller, Phys. Rev. B 54 (1996) 11169.

135. L. I. Granone, K. Nikitin, A. Emeline, R. Dillert, D. W. Bahnemann, Catalysts 9 (2019) 434.

136. D. Souri, Z. E. Tahan, Appl. Phys. B 119 (2015) 273.

137. M. G. Walter, E. L. Warren, J. R. McKone, S. W. Boettcher, Q. Mi, E. A Santori, N. S. Lewis, Chem. Rev. 110 (2010) 6446.

138. J. Wang, W. Cui, Q. Liu, Z. Xing, A. M. Asiri, X. Sun, Adv. Mater. 28 (2016) 215.

139. J. A. Turner, Science 285 (1999) 687.

140. H. Kisch, Semiconductor Photocatalysis: Principles and Applications, John Wiley \& Sons, Weinheim (2015).

141. L. Kantorovich, Quantum Theory of the Solid State: An Introduction, volume 136, Springer, Netherlands (2004).

142. C. Zou, W. Ji, Z. Shen, Q. Tang, M. Fan, Appl. Surf. Sci. 442 (2018) 778.

143. H. Guo, A. C. Marschilok, K. J. Takeuchi, E. S. Takeuchi, P. Liu, ACS Appl. Mater. Interfaces 10 (2018) 35623.

144. K.L. Salcedo Rodríguez, J.J. Melo Quintero, H.H. Medina Chanduví, A.V. Gil Rebaza, W. A. Faccio, R and Adeagbo, W. Hergert, C.E. Rodríguez Torres, L.A. Errico, Appl. Surf. Sci. 499 (2020) 143859.

145. G. S. Parkinson, Surf. Sci. Rep. 71 (2016) 272.

146. S. Trasatti, Pure Appl. Chem. 58 (1986) 955.

147. A. B. Murphy, P. R. F. Barnes, L. K. Randeniya, I. C. Plumb, I. E. Grey, M. D. Horne, J. A. Glasscock, Int. J. Hydrogen Energy 31 (2006) 1999.

148. J. R. Bolton, S. J. Strickler, J. S. Connolly, Nature 316 (1985) 495.

149. A. G. Hufnagel, K. Peters, A. Müller, C. Scheu, D. Fattakhova-Rohlfing, T. Bein, Adv. Funct. Mater. 26 (2016) 4435.

150. R. A. Henning, P. Uredat, C. Simon, A. Bloesser, P. Cop, M. T. Elm, R. Marschall, J. Phys. Chem. C 123 (2019) 18240.

151. K. Sivula, R. Van De Krol, Nat. Rev. Mater. 1 (2016) 15010.

152. D. K. Lee, D. Lee, M. A. Lumley, K.-S. Choi, Chem. Soc. Rev. 48 (2019) 2126.

153. N. Guijarro, P. Bornoz, M. Prévot, X. Yu, X. Zhu, M. Johnson, X. Jeanbourquin, F. Le Formal, K. Sivula, Sustain. Energy Fuels 2 (2018) 103.

154. T. Bredow, A. R. Gerson, Phys. Rev. B 61 (2000) 5194.

155. J. Chen, S. Shen, P. Guo, P. Wu, L. Guo, J. Mater. Chem. A 2 (2014) 4605.

156. Y. Huang, Y. Liang, Y. Rao, D. Zhu, J. J. Cao, Z. Shen, W. Ho, S. C. Lee, Environ. Sci. Technol. 51 (2017) 2924.

157. S. Al Khabouri, S. Al Harthi, T. Maekawa, Y. Nagaoka, M. E. Elzain, A. Al Hinai, A. Al-Rawas, A. Gismelseed, A. A. Yousif, Nanoscale Res. Lett. 10 (2015) 262.

158. J. H. Kim, Y. J. Jang, S. H. Choi, B. J. Lee, J. H. Kim, Y. Bin Park, C. M. Nam, H. G. Kim, J. S. Lee, J. Mater. Chem. A 6 (2018) 12693.

159. A. Arimi, L. Megatif, L. I. Granone, R. Dillert, D. W. Bahnemann, J. Photochem. Photobiol. A: Chem. 366 (2018) 118.

160. Z. Chen, H. N. Dinh, E. Miller, Photoelectrochemical Water Splitting, Springer, New York (2013). 
161. K. Rajeshwar, Encycl. Electrochem. 6 (2007) 1.

162. W. Schmickler, E. Santos, Interfacial Electrochemistry, Springer, Heidelberg (2010).

163. A. Pavese, D. Levy, A. H. Hoser, Am. Miner. 85 (2000) 1497.

164. F. Bræstrup, B. C. Hauback, K. K. Hansen, J. Solid State Chem. 181 (2008) 2364.

165. M. J. Akhtar, M. Nadeem, S. Javaid, M. Atif, J. Phys. Condens. Matter 21 (2009) 405303.

166. G. S. Y. Kumar, H. S. B. Naik, A. S. Roy, K. N. Narish, R. Viswanath, Nanomater. Nanotechnol. 2 (2012) 2012.

167. S. Nakashima, K. Fujita, K. Tanaka, K. Hirao, T. Yamamoto, I. Tanaka, Phys. Rev. B 75 (2007) 174443.

168. L. Yu, X. Zhang, G. Li, Y. Cao, Y. Shao, D. Li, Appl. Catal. B 187 (2016) 301. 